\title{
Addressing the Role of Health Literacy in Social Science: The Revision and Validation of the Perceived Oral Health Literacy Scale
}

Sara LaBelle

West Virginia University

Follow this and additional works at: https://researchrepository.wvu.edu/etd

\section{Recommended Citation}

LaBelle, Sara, "Addressing the Role of Health Literacy in Social Science: The Revision and Validation of the Perceived Oral Health Literacy Scale" (2014). Graduate Theses, Dissertations, and Problem Reports. 511. https://researchrepository.wvu.edu/etd/511

This Dissertation is protected by copyright and/or related rights. It has been brought to you by the The Research Repository @ WVU with permission from the rights-holder(s). You are free to use this Dissertation in any way that is permitted by the copyright and related rights legislation that applies to your use. For other uses you must obtain permission from the rights-holder(s) directly, unless additional rights are indicated by a Creative Commons license in the record and/ or on the work itself. This Dissertation has been accepted for inclusion in WVU Graduate Theses, Dissertations, and Problem Reports collection by an authorized administrator of The Research Repository @ WVU.

For more information, please contact researchrepository@mail.wvu.edu. 
Addressing the Role of Health Literacy in Social Science:

The Revision and Validation of the Perceived Oral Health Literacy Scale

\author{
Sara LaBelle \\ Dissertation submitted \\ to the Eberly College of Arts and Sciences \\ at West Virginia University \\ in partial fulfillment of the requirements for the degree of \\ Doctor of Philosophy in \\ Communication Studies
}

Keith D. Weber, Ed.D. Chair Melanie Booth-Butterfield, Ph. D.

Matthew M. Martin, Ph.D.

Scott A. Myers, Ph. D.

Melissa B. Wanzer, Ed.D.

Department of Communication Studies

Morgantown, West Virginia

2014

Keywords: Health Communication, Health Literacy, Intervention, Measurement, Validity Copyright 2014 Sara LaBelle 


\title{
ABSTRACT \\ Addressing the Role of Health Literacy in Social Science: The Revision and Validation of the Perceived Oral Health Literacy Scale
}

\author{
Sara LaBelle
}

The purpose of this dissertation was twofold. The first purpose was to develop a valid and reliable measurement of health literacy appropriate for use in social science. The second purpose was to determine whether health literacy is a skill set that can be increased through the intervention efforts of communication studies scholars. These purposes were addressed across four studies. The results of exploratory and confirmatory factor analysis provided support for a 26-item revised Perceived Oral Health Literacy Scale (LaBelle \& Weber, 2013) which assesses individuals' Motivation and Ability to gain access to, understand, and use health information in order to promote and maintain good health. Conceptually, this measure is consistent with the definition of health literacy put forth by the World Health Organization (2014), thus offering evidence of its content validity. Empirically, the results across the four studies provide strong evidence for the validity of the revised POHLS. Evidence for construct validity was provided by the measures positive relationships to patient self-efficacy, response efficacy and perceived health competence, while also being negatively related to trait and dyadic communication anxiety. Further evidence of construct validity was suggested by known groups differences in the POHLS scores obtained between individuals who had completed a semester long course in Health Communication and those who had completed a course in an unrelated topic. Evidence for the criterion- related validity of the measure was not supported, as the revised POHLS was not related to individuals' physiological indicators of health or stage of readiness to change behaviors related to obesity and diabetes prevention. The results of a pretest-posttest nonequivalent groups design did not provide support for the effectiveness of a brief educational intervention to increase individuals' health literacy; however, the known-groups assessment offers support to conduct further research on this topic. Taken together, these results provide support for the revised POHLS as a reliable and valid measure of health literacy appropriate for use in social science research. 
Dissertation written by

Sara LaBelle

B.A., Canisius College, 2010

M.A., West Virginia University, 2011

Ph.D., West Virginia University, 2014
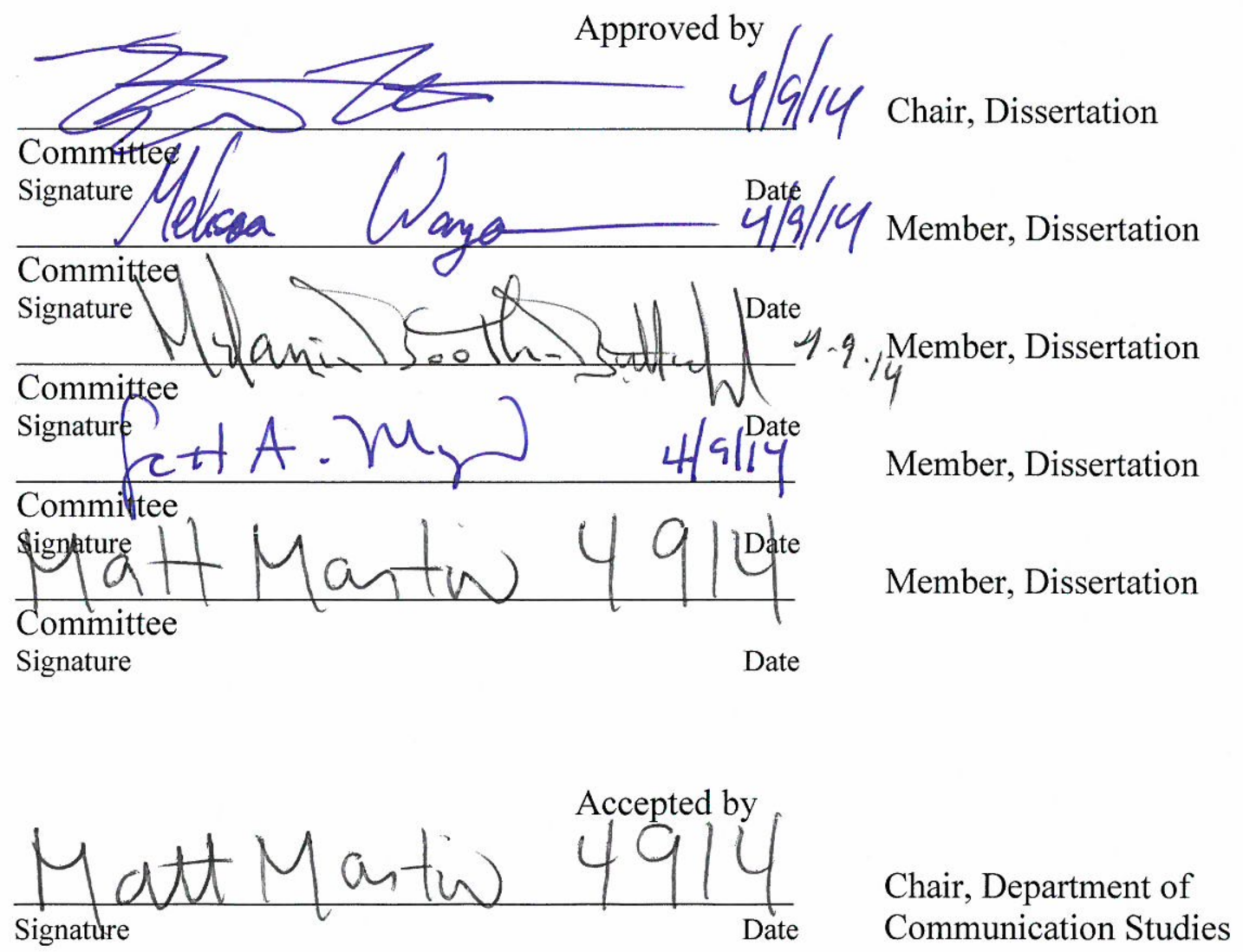
Page

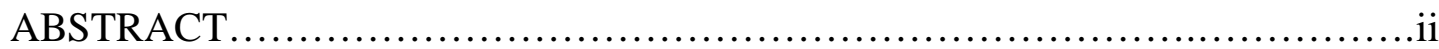

LIST OF FIGURES........................................................ viii

LIST OF TABLES ........................................................... ix

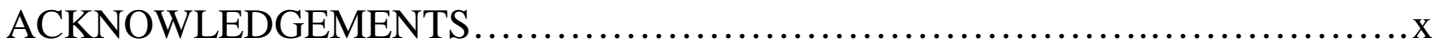

CHAPTER I: LITERATURE REVIEW ......................................1

Introduction.........................................................

Conceptualizing Health Literacy................................. 3

The Dimensions of Health Literacy........................6 6

The Importance of Studying Health Literacy............................. 11

Outcomes of Low Health Literacy................................13

Limited Knowledge and Understanding.................... 13

Poor Patient-Provider Communication....................... 15

Shame, Stigma, and Denial...............................17

Poor Treatment Adherence................................... 18

Adverse Health Outcomes................................ 19

Increased Health Care Costs and Hospitalization Rates........ 19

The Prevalence of Low Health Literacy........................... 20

The Measurement of Health Literacy.................................... 22

Narrow Assessment Range....................................... 25

Length of Assessment............................................26

Static Conceptualization of Health Literacy........................ 28

Problem Statement.................................................. 30

Rationale........................................................... 36

Research Question One...........................442

Content Validity................................................42

Hypothesis One...................................43

Construct Validity ............................................ 43

Perceived Health Competence.............................. 44

Hypothesis Two................................. 45

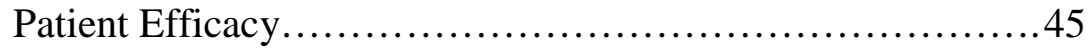

Hypothesis Three................................. 48

Communication Anxiety.................................. 48

Hypothesis Four................................. 49

Known-Groups Validity........................................49

Hypothesis Five................................ 51

Predictive Validity........................................... 51

Physiological Indicators of Health........................ 52

Hypothesis Six................................... 55

The Transtheoretical Model................................55 
Hypothesis Seven ..................................58

Increasing Health Literacy..................................... 58

Hypothesis Eight....................................60

Summary of Chapter I...................................................61

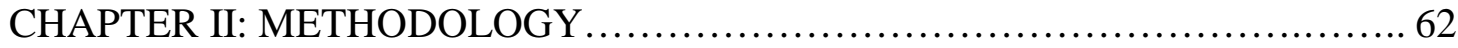

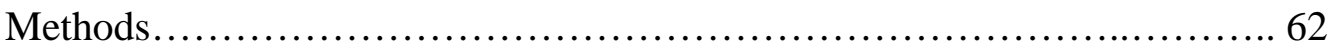

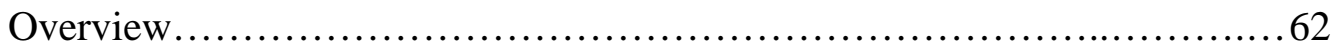

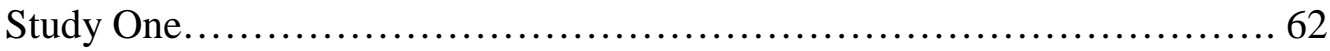

Participants and Procedures ......................................63

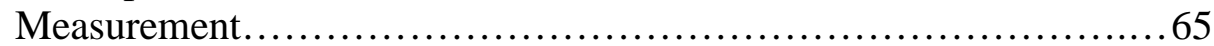

Health Literacy............................................65

Demographic Information..................................65

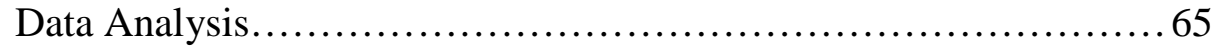

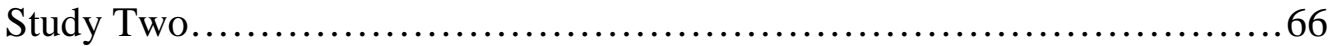

Participants and Procedures .......................................66

Measurement ...................................................68

Health Literacy............................................68

Perceived Health Competence..................................69 69

Patient Efficacy ..........................................69

Communication Anxiety....................................70

Demographic Information.................................71

Data Analysis ................................................. 71

Study Three............................................................ 72

Participants and Procedures ..................................... 73

Measurement ................................................. 74

Health Literacy...........................................74

Demographic Information...............................74

Data Analysis .................................................. 74

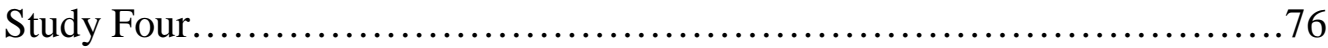

Participants and Procedures ..................................... 77

Measurement ................................................ 79

Health Literacy.........................................79

Physiological Health Indicators.............................. 79

Stages of Change........................................... 80

Demographic Information...................................83

Data Analysis ................................................... 83

Summary of Chapter II................................................. 83

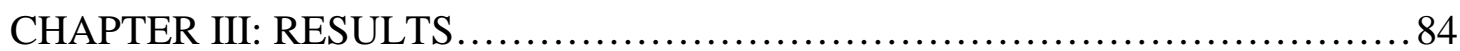

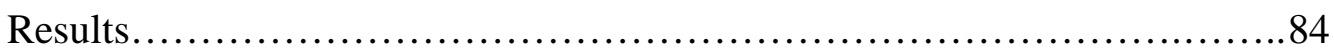

Results of Research Question One.................................84

Results of Hypothesis One......................................... 89

Results of Hypothesis Two........................................ 97 
Results of Hypothesis Three..................................... 98

Results of Hypothesis Four......................................99

Results of Hypothesis Five..................................... 100

Results of Hypothesis Six...................................... 100

Results of Hypothesis Seven........................................100

Results of Hypothesis Eight..........................................101

Summary of Chapter III............................................. 103

CHAPTER IV: DISCUSSION............................................... 105

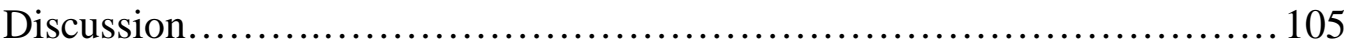

Scale Development............................................... 105

Validity Assessments...................................... 110

Increasing Health Literacy..................................... 116

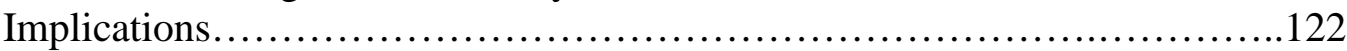

Limitations and Future Directions.............................. 129

Conclusion............................................................ 133

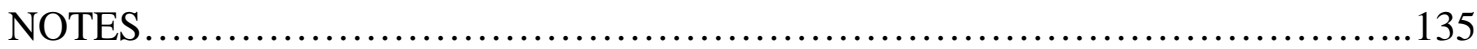

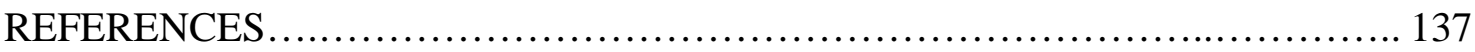

APPENDICES................................................................. 173

Appendix A: The Rapid Estimate of Adult Literacy in Medicine (REALM). 173 Appendix B: The Rapid Estimate of Adult Literacy in Medicine Short Form

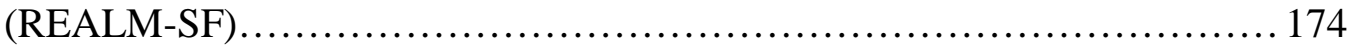

Appendix C: The Short Test of Functional Health Literacy in Adults

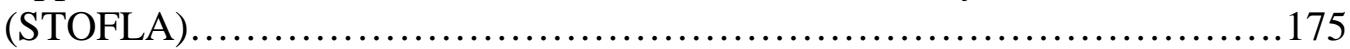

Appendix D: The Newest Vital Sign (NVS).............................177

Appendix E: Original Perceived Oral Health Literacy Scale.................... 179

Appendix F: Pilot Test Questionnaire.......................................180

Appendix G: Item Pool Used in Study One..................................185

Appendix H: Study One Recruitment Script............................. 188

Appendix I: Study One Cover Letter.................................... 189

Appendix J: Study One Questionnaire..................................... 190

Appendix K: Revised Perceived Oral Health Literacy Scale..................... 195

Appendix L: Study Two Phase One Recruitment Script........................ 197

Appendix M: Study Two Phase One Cover Letter............................. 198

Appendix N: Notes for Educational Intervention .............................. 199

Appendix O: Study Two Phase Two Recruitment Script...................... 201

Appendix P: Study Two Phase Two Cover Letter.............................202

Appendix Q: Perceived Health Competence Measure...........................203

Appendix R: Patient Self and Response Efficacy Measure.....................204

Appendix S: Form Trait Subscale of the Communication Anxiety Inventory. 205

Appendix T: Study Two Phase One and Phase Two Questionnaire............206

Appendix U: Study Three Recruitment Script.................................2214

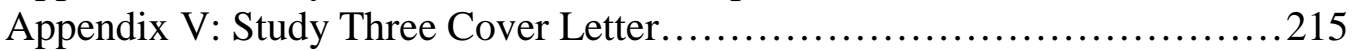


Appendix W: Study Three Questionnaire...............................216

Appendix X: Study Four Recruitment Script..............................221

Appendix Y: Study Four Recruitment Flyer ............................2222

Appendix Z: Study Four Consent Form................................... 223

Appendix AA: Study Four Cover Letter.................................226

Appendix BB: Stages of Change Measure............................... 227

Appendix CC: Physiological Health Indicators..........................228

Appendix DD: Study Four Questionnaire .................................229 


\section{LIST OF FIGURES}

Page

Figure 1. Baker’s Conceptual Model of Health Literacy...............................10

Figure 2. Scree Plot for Exploratory Factor Analysis.................................85

Figure 3. Four Factor Measurement Model....................................... 93

Figure 4. Two Factor Measurement Model........................................... 95 


\section{LIST OF TABLES}

Table 1.Original Item Pool for Perceived Oral Health Literacy Scale................. 37

Table 2: EFA Factor Loadings for Perceived Oral Health Literacy Scale.............. 39

Table 3: Study Two Demographic Data Categorized by Experimental Condition....... 67

Table 4. Study Three Demographic Data Categorized by Group......................75

Table 5: Frequency of Stages of Change for Physical Activity and Healthy Eating......82

Table 6: Factor Loadings for Four Factor Solution............................. 90

Table 7: Standardized Regression Weights for Four Factor Model.................. 94

Table 8. Standardized Regression Weights for Two Factor Model...................96

Table 9: Correlations Between Health Literacy Factors, Perceived Health Competence, Patient Efficacy, and Communication Anxiety................................. 99

Table 10: Motivation and Ability Scores for Experimental and Control Group at Pretest and Posttest.................................................................... 102 


\section{ACKNOWLEDGEMENTS}

I will admit, I have thought about what it would be like to sit down and write this page many times as I have made my way through the past three years. At some points, the fact that I would be sitting here, having made it through all of the reading, the writing, the late nights, the tears, the stress, the panic, the "I can’t do this" moments... the fact that I am sitting here on the other side of writing and defending my dissertation, as Dr. LaBelle... well, I didn’t always think I would make it. It will come as absolutely no surprise to those who know me well that the past three years, four if you count my Master's degree, have been a challenge for me. But this page isn't about me... it is about all of the people who have allowed me to sit here, after all of the worrying and stress, and write my acknowledgements page for my dissertation.

I am going to start my long list of "thank yous” with something that might seem generic to others, or even clichéd, but I absolutely cannot thank one single person before I thank God for allowing me to be here. Moving away from my family and figuring out what I want to do with my life has required so much of my faith, and the strength and peace I find in prayer has never failed to carry me through my struggles.

My graduate career began in August 2010, at which point I moved to West Virginia, away from my entire family and everything that was familiar and comfortable. The transition was very fast and very overwhelming, and I was absolutely scared out of my mind. The very first night I came home from my classes, I called my Dad and told him I couldn’t make it- I was way out of my league, not smart enough, and would be quitting at some point very soon. My Dad's response was one that I have never forgotten:

"You will NOT quit, because that is not who you are... Say a prayer, toughen up, and go 
get them, Rocky.” At various points over the past four years, my Dad must have repeated that line a few hundred times in some variation, and "Rocky” has become a regular nickname. To my Dad: I could never, ever come up with the right words to thank you for all that you have done for me. You have supported me in every way possible, believed in me when I didn’t believe in myself, and helped me to realize that the only thing stronger than fear is faith. I am so incredibly blessed to have a Dad who loves me as much as you do. Thank you.

To my Mom: I honestly don’t know what I would have done without my “mom lifeline” phone calls throughout these past few years. I don't think anyone in the world can listen to me complain, cry, vent, swear, yell, talk myself down, laugh, and repeat the process over again like you do! And you always end the conversation by encouraging me, letting me know that I will figure it out "because I always do,” and giving me the strength I need to push forward. I believe in myself because you believe in me. You have been 100 percent by my side through every up and down moment over these past few years, and I know that I have my first and best friend by my side for whatever the future holds. I see myself becoming more of your "mini me" all the time (except the great cooking, so if you could have eased up on the sarcasm gene and put a little more "I can make anything taste like heaven” in me that would have been cool), and I couldn’t be more proud, because you truly are my idol. Thank you so much, Mom.

To my brothers, Tommy and Jon. I have spent the better part of my life looking up to you (as soon as I got over wanting to punch you in the face all the time for teasing me, Jon), and you will always be my heroes. Tommy, you taught me to do what I do with passion. Jon, you convinced me at a very young age that I loved homework so much that 
I wanted to do yours as well $\odot$ I I might be the one with the $\mathrm{PhD}$ now, but I am convinced you are both far more intelligent and talented than I could ever be. I love you guys, and I could never ask for two better big brothers. To Nicole and Jacqui- thank you for being more like sisters than sisters-in-law. I could never ask for a better group of people to call my siblings.

Anna, Anthony, Dominic, Leo, Nicholas, Olivia, and Taylor. This "thank you” is truly more of an "I’m sorry”- I am sorry for missing birthdays, holidays, first words, school plays, and for countless other times I wish I could have been there. I hope you know that I have carried all of you in my heart throughout this entire journey. I did this in a way FOR you- maybe someday when you are on the verge of a big, scary, uncertain opportunity, you feel more confident in taking that first step because of your Aunt Sara.

To my brother-cousins, Mark and Jay- I think the hardest part of being in WV has been missing out on laughing hysterically with you guys all the time. I am so glad that God gave me a second set of brothers to look up to and feel protected by. Thank you for all of the messages, the support, and the "me missing you while doing laundry/cooking/camping” pictures. Father Diguilio, Colleen, Debbie, Tracy, Wendy, Jessica, Aunt Suzi, Uncle Mark and Aunt Jacqui, Uncle Paul and Aunt Becky, Aunt Annie, Uncle Anthony and Aunt Laura, and to ALL of the 31 cousins and second cousins that have supported me and encouraged me along the way- THANK YOU!

To Dr. Weber, I almost feel weird thanking you here because there is absolutely nothing I could say that would do justice to all that you have done for me as an advisor, mentor, and friend. You helped me to grow up in my time at WVU, and figure out who I am not just as a researcher but as a person. Next year, I KNOW there will be a time (or 
more likely several hundred times) when I am walking around Chapman and think "I wish I could go talk this out with Dr. Weber.” You have pushed me to do things I would have NEVER believed I could do (seriously what the hell did you have me take that stats class for), taught me what it means to be a good teacher (although I will never be as good as you are), and given me everything I need to make my own mark on the world. If I had to make the decision of who would be my advisor 100 more times, I would choose you every time. Also please know that me leaving WVU does not mean I will stop bothering you- knocks on the door will just become emails. And texts. And calls. :-) INDEED! I consider myself truly fortunate to have been taught and molded by the faculty of WVU. Dr. Martin, thank you for giving me SO many opportunities and for having so much confidence in me as a student. Earning your accolades has been one of my greatest accomplishments. Dr. Myers, thank you for scaring the living sh*t out of me until it made me a better writer and researcher. This past year you have given me so much guidance, and I will really miss the opportunity to stop in your office to ask "How do I write this so that I don’t sound like an idiot?” next year () Dr. Booth-Butterfield, thank you for always pushing me to think harder about my research. You are such an inspiration to me, and your endless support and encouragement have given me so much comfort over these past few years. Dr. Wanzer, how do I thank the person who inspired my entire career? I will never forget the first few moments I had you as my Professor back in Fall 2007 and thinking "I want to be like her" - I will always look up to you as a teacher and a mentor in that way. You treat every student like they are something special and your students never forget it. I would never have dreamt I could do this if you hadn’t put it in my head seven years ago- and your support and faith in me has made such a huge, huge difference 
in who I am today. Thank you to my entire committee for being the best group of faculty I could ever ask to work with on a dissertation.

To all of the faculty and staff at WVU, thank you for helping me to get to this point. A special note to Dr. Goodboy in particular to always talking me up like I am a superstar- that means a lot to us insecure folks $;$; I am so happy to have made you proudand I hope I keep it up. Terri, I literally would have never survived the copier or the paperwork without you! You are a miracle worker. Joy, thanks for treating every grad student who is freaking out over something stupid like they are important and always stopping to help- we notice. Renee, I am going to miss you so much next year. I always feel "at home” when I walk into 108 and can vent to you three ladies. Also, thank you for helping me with the earlier parts of this dissertation- I know you are all swamped with work all the time so that meant a lot. John Cole, thanks for calling me before and after every big, stressful meeting- Your genuine support and concern about how I was doing was always so humbling. There were many people who helped me by either allowing me to come into their classes, by helping recruit participants for my studies, or helping me out with all of the small (but hugely important to me) "to dos" along the way. For this I thank Alex, Annie, Mary, Jessalyn, Dr. Brann, Dr. Cohen, Akeya, and Suzanne (and all of the wonderful people at The Shack). A special thank you to Lesli- as my research assistant, you not only helped to save my surveys from spontaneously combusting stairs and suddenly appearing pools of water that would destroy my data, but (perhaps more importantly) reminded me to quit taking things so serious and to have fun along the way.

"No man is a failure who has friends"- My friends have celebrated with me when I was successful, and been there for me when I was not. To my best friend of 12 years, 
Nicole- being my best friend has not been easy these past few years (or ever, really), but you have truly stood the test of time as a true, honest, and loyal friend. I know that you will be there for every up and down on the road ahead, and I can't wait to see what adventures we go on next. Hannah, for someone I have known for such a short amount of time, I can’t even put into words how much I appreciate your friendship. Without you, I would have been a complete train wreck this year (as opposed to the partial train wreck I actually was). You truly are one hell of a nose. To Jojo, Amy, Kara, Allyson, and Megan- I am so lucky to have a group of such strong, beautiful, independent, and successful women to call my best friends. I love you all so, so much.

I don't know how I would have made it through either degree without my WVU family of friends. Michelle, Tiff, Annemarie, Angel, Grant, and Kelly- thank you for continuing to support me while I stuck around Armstrong Hall a little longer ;) To Michael, thank you for being my friend and cheerleader (a very manly cheerleader, of course) for three years. It has meant the world to me that you have always fully supported me, even when you had your own graduate career to worry about! I would have been lost this year if I didn't have you to confide in. An extra special thank you to my friends who supported me and cheered me on this year: Zac (“Littlefoot”), Greg (“Eagle!”), CJ, Alex, Shannon, Melissa, Jordan, Shaun, Rita, Denise (DE-NICE), Betsy, and Hailey.

Finally, to my Zac. I don't even know how to thank you for all that you have done for me over the past few years. You have been my best friend, my supporter, my sounding board, my workout buddy, my inspiration, and pretty much my therapist over the past few years :- If you had a dime for every time I asked you "will everything be okay?” we would both be rich! I am sorry to be such a pain in the ass to date, but you are 
stuck with me now $:$ Giving in and going out to get Mexican food with the cute (and persistent) mustached-doc student was the best decision I ever made. I also have to thank your amazing family for sending me so many notes, cards, letters, and messages- I really lucked out with them. I love you to the moon and back (and to Kentucky to West Virginia and back... and to California and back... to WHEREVER and back), and I am SO excited for what our future holds.

To any graduate student that might come across this, hoping that one day they will get the chance to write their own acknowledgements page, I have two pieces of advice: 1 . Realize that graduate school is not a one person accomplishment. Take a second to think about all of the people helping you reach your goals right now, and let them know you appreciate them. This includes the people who are earning the same degree with you- they aren’t competition, they are company. Be genuinely happy for them when they succeed- and remember that a success for them does not constitute a failure on your behalf. 2. When everything gets overwhelming and you feel like quitting, take a BIG breath and think about this: if your biggest worry right now is that you won't do well in a PhD program, you are lucky as hell. Go for a run, do some yoga, pet a cat, do whatever you need to calm down and realize how small you are in a big, big, world... and then get your s\#@t together and do the best you can.

Thank you again, to all of the people that helped me get here.

Sara

p.s. I am also going to thank my cat, Sergeant Judy Biscuits. I thought I was rescuing her, but she is the one who saved me. In honor of her, please adopt, don't shop :- 


\section{CHAPTER I}

\section{Introduction}

The field of health communication has produced a sound body of research on the communicative challenges, solutions, and complexities inherent in public health development and delivery across multiple channels, contexts, and levels of healthcare (Kreps, 2001; Kreps, Bonaguro, \& Query, 1998). Undoubtedly a very broad and complex area of study, health communication scholars study a range of contexts and issues including but not limited to preventative healthcare, the physician-patient relationship, health information seeking, and the development and implementation of communication campaigns (see Thompson, Parrott, \& Nussbaum, 2011). The field has been conceptualized as having two distinct and interdependent branches: healthcare delivery systems and healthcare promotion (Kreps et al., 1998). As described by Kreps and colleagues (1998), the study of healthcare delivery systems encompasses the role of communication in patient-physician communication and healthcare information systems and the study of healthcare promotion focuses on the persuasive use of communication messages and media to promote public health. Research in these branches has produced an impressive body of knowledge on effective means of communicating with patients across varying cultural backgrounds (Kline, 2007), types of disabilities (Bute, DonovanKicken, \& Martins, 2007), and phases of the illness processes (Brann, Himes, Dillow, \& Weber, 2010; Bute \& Vik, 2010; Weber \& Solomon, 2008), as well as the influence of social support (Haas, 2002) and effective campaign strategies for both interpersonal and mediated channel delivery (Hwang, 2012).

The nature of research and practical application in both of these branches is 
changing rapidly with drastic increases in the availability and depth of health information provided to the public. Each day, individuals are inundated with health messages from physicians, pharmacists, television and radio advertisements, and the nearly endless amounts of information available on the Internet. As phrased by Bernhardt and Cameron (2003), however, the utility and effectiveness of this widespread health information is severely lessened, if not reversed, if individuals are not able to fully access, understand, and apply it correctly. As such, it is of critical importance for health communication scholars to begin to more fully consider the role of health literacy in its impact on health communication in both healthcare delivery systems and healthcare promotion. Indeed, health literacy presents a public health issue relatively untouched in previous health communication inquiry, despite its widespread influence on both "branches" of the field. In setting the agenda for research to be published in the field of communication studies’ premier health journal, Health Communication, Kreps (1989) argued that the greatest value of health communication inquiry is the vast potential it has to be applied to pressing social issues in order to make significant change. As stated by Kreps, "the primary goal for health communication is not to break out in print but to generate health communication knowledge for directing healthcare policy, practice, and intervention” (p. 14-15). This sense of responsibility to the public welfare has generated nearly 25 years of research applying theories of health communication to achieve attitudinal and behavioral change (e.g., Hwang, 2012; Noar, 2006; Rogers, 2004; Valente, 1996). Given this responsibility, it is imperative that health communication researchers and practitioners devote more attention to the role of health literacy in the health information delivery and reception process. Approximately 80 million adults in the 
United States struggle with low health literacy, or the skills necessary to function effectively in the healthcare environment (Berkman, Sheridan, Donahue, Halpem, \& Crotty, 2011), yet healthcare practitioners and researchers have overlooked, until just recently (For a review of the milestones achieved in health literacy research and reform, see Parker \& Ratzan, 2010), the influence of this construct (Bernhardt \& Cameron, 2003). In the sections that follow, a definition and overview of research and scientific knowledge on health literacy will be provided. This review of literature will be followed by a discussion of the issues facing social science researchers regarding the measurement of health literacy and a proposed research study to address these concerns.

\section{Conceptualizing Health Literacy}

As highlighted by several scholars examining the construct (Baker, 2006;

Berkman, Davis, \& McCormack, 2010; McCormack, Haun, Sorensen, \& Valerio, 2013;

Parker \& Ratzan, 2010), an increasing research focus on health literacy has been accompanied by an equal amount of confusion and disagreement on how to conceptualize it. As defined by the National Literacy Act in 1991, literacy constitutes an individual's ability to read, write, and speak in English; to compute and solve problems at sufficient levels of proficiency to function socially and at work; to achieve one's goals; and to develop his or her knowledge and potential. In the 1990s, healthcare professionals and practitioners began to discuss the possibility of a more specific type of literacy, health literacy (Bernhardt \& Cameron, 2003). Notably, scholars in communication studies have long adapted the general concept of literacy into specialized contexts such as media (Hobbs, 1998; Livingstone, 2004; Potter, 2012) and information (Behrens, 1994; Webber \& Johnston, 2000) literacy. The American Medical Association’s (AMA) Ad Hoc 
Committee on Health Literacy (1999) defined this “new” construct as "the ability to read and comprehend prescription bottles, appointment slips, and other essential health-related materials required to successfully function as a patient” (AMA, 1999, p. 552).

The conceptualization of health literacy as one's ability to read and comprehend information in the medical context has been referred to as a functional definition of health literacy, as it reflects one’s literacy within a particular context (Nutbeam, 2000). Definitions developed from this perspective focused on patients' ability to read consent forms, health education materials, and insurance applications (Parker, Williams, Baker, \& Nurss, 1996; Tuckson, 2004). As much of the early research on health literacy was conducted by medical professionals, it should not be surprising that these conceptualizations of health literacy were heavily clinician-oriented and situational (Bernhardt \& Cameron, 2003).

However, as Nutbeam (2000) contended, these functional (and admittedly narrow) definitions of health literacy do not account for the deeper influence and purpose of literacy in everyday life. While the reading and writing skills comprised in these definitions may allow for sufficient functioning in the healthcare environment, they do not account for those skills which allow individuals to actively participate in their healthcare and derive their own meanings and interpretation from exposure to health information (Nutbeam, 2000). In contrast, communicative or interactive definitions of health literacy focus on the more advanced cognitive and social skills which allow individuals to adapt new health information to their own lives. Even further, definitions which focus on the critical aspect of health literacy account for an individual's ability to apply advanced cognitive skills (in combination with social skills) to critically analyze 
health information and exert higher amounts of control over health situations (Nutbeam, 2000).

Employing a broader definition of the construct than the functional perspective, the Healthy People 2010 report defined health literacy as an individual's capacity to obtain, interpret, and understand basic health information and services, along with the competence to use the information and services to improve one's health (U.S. Department of Health and Human Services, 2000). In a similar vein, Berkman and colleagues (2010) defined health literacy as "the degree to which individuals can obtain, process, understand, and communicate about health-related information needed to make informed health decisions” (p. 16). Similarly, the IOM (2004) report defines health literacy as "the degree to which individuals have the capacity to obtain, process, and understand basic health information and services needed to make appropriate health decisions” (p.5). Thus, contemporary definitions of health literacy reflect that an individual's level of health literacy is not only a function of his or her ability to read and write, as the initial clinician-based definitions suggested, but is also comprised of a set of unique social skills and capabilities enabling an individual to actively succeed in health decision-making processes (Bernhardt \& Cameron, 2003; Nutbeam, 2000). Of key importance to health communication scholars is the emphasis on examining the communicative aspects of the health literacy phenomenon.

The World Health Organization (WHO; 2014) defines health literacy as "the cognitive and social skills which determine the motivation and ability of individuals to gain access to, understand and use information in ways which promote and maintain good health” (“Track 2: Health literacy and health behaviour,” para. 1). This definition will be 
used to frame and understand health literacy throughout the remainder of this study. There is no one "correct" definition of health literacy; rather, the definition that researchers or practitioners choose is largely reflective of their goals (Berkman et al., 2010). The WHO definition was therefore selected as it acknowledges that although health literacy is certainly a function of reading and numeracy abilities, it is also manifested in and affected by the communication of health messages. In contrast to functional definitions of health literacy, the WHO definition reflects both communicative/interactive and critical aspects of the construct (Nutbeam, 2000).

The WHO definition also presents health literacy as dynamic rather than static or fixed. A static or fixed conceptualization of health literacy would imply that individuals' ability to process health information remains relatively stable across adulthood, whereas a dynamic conceptualization allows the possibility for individuals' levels of health literacy to change and be improved through experience and interventions (Berkman et al., 2010). A static definition of health literacy is an artifact of its origins in being assessed as prose literacy and the limitations of initial measurements (Berkman et al., 2010). Increased sophistication in the field and in corresponding measurement necessitates a dynamic measurement of the construct. As stated by Nutbeam (2000), the WHO definition implies that the personal and social benefits of health literacy might be improved by health education and communication.

The dimensions of health literacy. In accordance with the WHO (2014) definition of the construct, there are two dimensions of health literacy examined in this manuscript: cognitive and social. The cognitive aspect of health literacy has become an umbrella term to represent at least four distinct components (Bernhardt \& Cameron, 
2003). The first component of cognitive health literacy is reading literacy, or the ability to interpret words and their meanings, use and recognize a wide range of words, find meanings for uncommon words, scan visuals to find key concepts, and separate key points from details (Doak, Doak, Friedell, \& Meade, 1998). The second component of cognitive health literacy is numeracy, which reflects an individual's ability to understand and interpret numbers (Golbeck, Ahlers-Schmidt, Paschal, \& Dismuke, 2005; Schwartz, Woloshin, Black, \& Welch, 1997). Numeracy is a particularly crucial aspect of health literacy as so much of the health information provided to patients requires the ability to read, understand, and apply numbers (e.g., medical directives; Bernhardt \& Cameron, 2003; Schwartz et al., 1997).

The third component of cognitive health literacy is media literacy or the ability to access, analyze, evaluate, and produce messages in a variety of communication channels, as well as to assess the true value and meaning behind mediated messages (Austin \& Johnson, 1997; Potter, 2012; Zettl, 1998). The fourth component of cognitive health literacy is computer literacy which reflects the changing nature of health information seeking in the technological age (Bernhardt \& Cameron, 2003). Individuals who are not able to use and interpret the massive amount of health information available on the Internet are at a drastic disadvantage in the modern age (Bernhardt \& Cameron, 2003). Overall, the abilities that individuals have in reading, numeracy, media, and computer literacy reflect the cognitive aspect of what constitutes health literacy; undoubtedly, successfully functioning in the healthcare environment requires one to be able to access, process, understand, analyze, and apply health information using these varied skills. However, of particular interest to health communication scholars is the complementary 
and distinctly communicative aspect of the health literacy phenomenon: the social side of health literacy.

Health literacy also reflects the social skills required to interact and communicate effectively with healthcare providers or other members of the healthcare system (e.g., insurance providers, health management organizations, technicians, secretaries) (Bernhardt \& Cameron, 2003). Referred to as communicative or interactive literacy by Nutbeam (2000), this dimension of health literacy represents the more advanced social skills which can be used to actively participate in healthcare, to derive meaning from various forms of communication, and to apply new information to new situations and circumstances. To communicate effectively in the health environment, an individual must be able to clearly articulate physical, mental, and emotional states to healthcare representatives (Bernhardt \& Cameron, 2003). Individuals should be able to do this under a variety of situations, including emergencies, with people in powerful positions, and when badly injured or under time pressures. The ability to communicate effectively is a crucial aspect of not only accessing and applying health information, but also how well that information is applied by both members of the healthcare system and the individual (Bernhardt \& Cameron, 2003).

In an attempt to streamline and create consistency among the various conceptualizations of health literacy and its domains, Baker (2006) forwarded a conceptual model of health literacy which outlines not only the two distinct aspects (health-related print literacy and health-related oral literacy) of the construct, but also their antecedents and relationship to various outcomes. The first major domain represented in the model is individual capacity, or the set of resources one has to deal 
effectively with new health information, personnel, and the healthcare system in general. As Baker highlights, there are two sub-domains of individual capacity: reading fluency and prior knowledge. Reading fluency is the ability to process written materials and form new knowledge, and is composed of prose, document, and quantitative ability. Prior knowledge, or the amount of knowledge one has prior to being exposed to health information or speaking to a healthcare professional, is comprised of both vocabulary (i.e., knowing the meaning of individual words) and conceptual knowledge of health and healthcare (i.e., understanding aspects of the world, such as how the body functions). These two sub-domains are related in that reading fluency allows one to expand upon vocabulary and conceptual knowledge, which in turn allows one to more easily consume health related information from various sources and therefore build on their prior knowledge for anticipated exposure to health-related information and interactions with healthcare professionals. The mutual influence among these constructs is illustrated in the covariance paths of the model (see Figure 1).

The second major domain of Baker's (2006) model is health literacy, which is divided into print literacy and oral literacy in accordance with the IOM report on the construct. As indicated in the model, both of these health literacy dimensions are impacted by the individual capacity (i.e., reading fluency and prior knowledge) discussed above, as well by system factors such as the complexity and difficulty of printed and spoken messages. These health literacy dimensions (i.e., health-related print and oral literacy) are in turn posited to be one of many factors, such as culture and barriers to change, that lead to knowledge acquisition, behavior change, positive health behaviors, 
Figure 1

\section{Baker's Conceptual Model of Health Literacy}

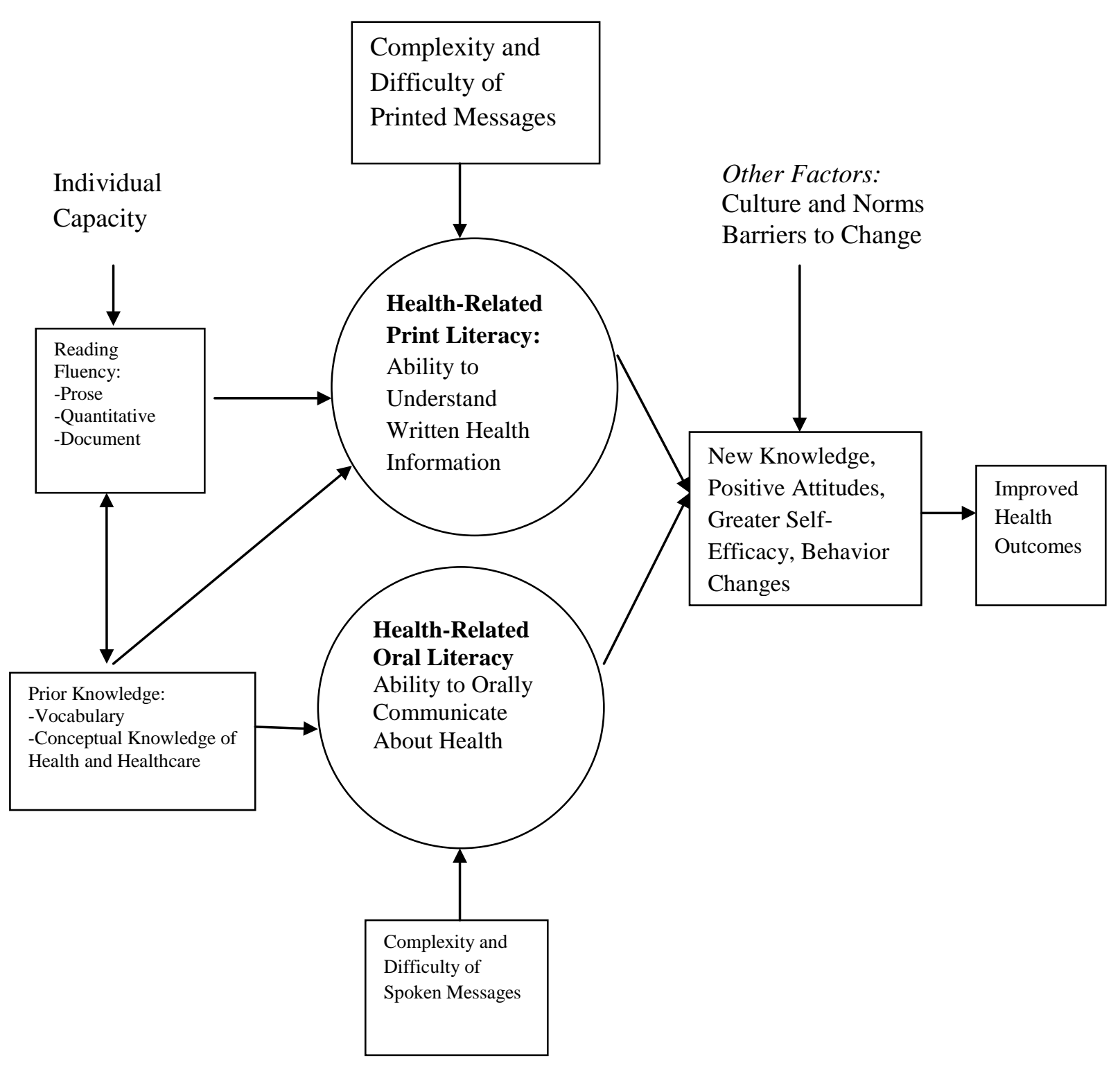


and other indicators of success in the healthcare environment. Baker's model is important in its equal emphasis on the individual and system-level influences on both health literacy and its associated outcomes such as increased knowledge, attitude formation and alteration, and behavior change (Baker, 2006; Cameron, Wolf, \& Baker, 2011). As stated in Healthy People 2010, health literacy is dependent on both individual and system factors, including the communication skills and knowledge of lay persons and professionals, culture, the demands of the healthcare system, and the particular context (Berkman et al., 2010).

\section{The Importance of Studying Health Literacy}

In the early 1990s, research on health literacy was largely conducted at the national level (by the Department of Health and Human Services), and focused on defining the construct and establishing its prevalence (Parker \& Ratzan, 2010). The work conducted on these efforts is published in the 2004 Institute of Medicine (IOM) report, which concludes "efforts to improve quality, reduce costs, and reduce disparities cannot succeed without simultaneous improvements in health literacy” (IOM, 2004, p. xi). Low health literacy has evolved from an under-recognized and under-researched "silent epidemic” to an issue of health policy and reform in recent years (Parker \& Ratzan, 2010, p. 21). Thirteen institutes and centers of the National Health Institute (NIH) and the Agency for Healthcare Research and Quality (AHRQ) issued a Program Announcement for Review (PAR) starting in 2004 (which was reissued in 2007 and 2010), with the goal of increasing the nature of health literacy and its association and impact on health outcomes. In 2009, the IOM Health Literacy Roundtable hosted the first annual Health Literacy Research Meeting to present and discuss federally funded research findings on 
health literacy and to discuss future directions for research (Parker \& Ratzan, 2010).

Louisiana was the first state to include legislation to improve the low health literacy issues of the state in 2003 with other states following during the next 10 years. Senators Norm Coleman and Thomas Harkin co-sponsored the National Health Literacy Act in 2007 and health literacy provisions were included in the House and Senate bills of the $111^{\text {th }}$ Congress on health reform. The Patient Protection and Affordable Care Act, P. L. 111-148 signed by President Barack Obama on March 23, 2010 includes a definition of health literacy (i.e., an adopted definition from the IOM and Healthy People 2010) and integrates the improvement of health literacy into healthcare reform. Specifically, the Act mandates dissemination of research on health literacy, improved prescription medication labeling for individuals with low health literacy, and the education of healthcare providers on the prevalence and amelioration of low health literacy (Parker \& Ratzan, 2010).

As evidenced by these federal and legal developments, it has become a national priority to conduct further research on health literacy (Benjamin, 2010; Parker \& Ratzan, 2010; Ratzan, 2013). A major facet of the impact health literacy has on individuals' health outcomes is the communicative exchange of healthcare practitioners and lay persons (Nutbeam, 2000). Low health literacy has consistently been shown to be related to poorer health outcomes and decreased patient satisfaction (Berkman et al., 2011). Professionals in healthcare settings often mistakenly assume that patients are able to read educational brochures, prescription labels, and written instructions, and are unaware that many adult patients with low literacy skills will actively hide their reading deficiencies due to embarrassment and stigma (Murphy, Davis, Long, Jackson, \& Decker, 1993; 
Williams, Davis, Parker, \& Weiss, 2002). In the following section, the extant body of research knowledge on the antecedents, outcomes, and overall impact of health literacy on individuals’ communicative, psychological, and physical health will be reviewed.

\section{Outcomes of Low Health Literacy}

It is estimated that issues related to low health literacy and the subsequent miscommunication between providers and patients costs between 106 billion and 238 billion dollars annually (Vernon, Trujillo, Rosenbaum, \& DeBuono, 2007). In addition to these financial costs, low health literacy has a number of negative physical and psychological effects on individuals. It is consistently associated with more hospitalizations, greater use of emergency care, and poorer ability by patients in demonstrating how to take medicines and in interpreting health messages (An \& Muturi, 2011; Berkman et al., 2011; Lanning \& Doyle, 2010; Shone, King, Doane, Wilson, \& Wolf, 2011; Weiss \& Palmer, 2004). Individuals with low health literacy also experience challenges in self-managing chronic health conditions, such as diabetes and hypertension (Osborn, Cavanaugh, Wallston, \& Rothman, 2010; Osborn et al., 2011; Schillinger et al., 2002; Williams, Baker, Honig, Lee, \& Nowlan, 1998; Williams, Baker, Parker, \& Nurss, 1998). Research on health literacy has identified six major negative outcomes associated with lower levels of health literacy (Bernhardt \& Cameron, 2003). Evidence on the association of health literacy and these outcomes is provided in the next sections.

Limited knowledge and understanding. Limited health knowledge has been the most widely identified outcome in research on health literacy (Paasche-Orlow \& Wolf, 2010). This includes decreased knowledge of disease and prevention strategies as well as less informed medical decision making (Davis et al., 1996; Morris, Field, Wagner, 
Cutrona, \& Roblin, 2013; Ussher, Ibrahim, Reid, Shaw, \& Rowlands, 2010). In a study on 5,929 patients across thirteen healthcare organizations nationwide, Wynia and Osborn (2010) found that patients who reported literacy challenges also reported problems learning about their own medical conditions due to not understanding written information, a lack of confidence in completing medical forms alone, and needing someone to assist them in reading hospital and clinic materials (Wynia \& Osborn, 2010). In a study by Davis and colleagues (1996) on the relationship of literacy and mammography screening, the researchers found that $39 \%$ of the women in their sample (aged 40 and older in a mammography clinic) admitted to not knowing why women get mammograms. Of the women with low health literacy who said that they did know why, many provided inaccurate reasoning for this preventative health behavior. Individuals with low health literacy are also more likely to have a fatalistic attitude toward cancer, less able to identifying the purpose of cancer screening tests (Morris et al., 2013), and (even when insured) less likely to be up to date on screening for colorectal cancer (Morris et al., 2013; Sentell, Braun, Davis, \& Davis, 2013). Notably, the participants in Morris et al. (2013) were less likely to have met the appropriate guidelines for colorectal screening even when insured.

In a study on management of hypertension and diabetes, Williams et al. (1998) found that individuals with low health literacy did not have a sufficient amount of knowledge regarding their disease, how to manage it, and the lifestyle modifications that they needed to make. Misunderstandings attributed to low health literacy have also been found with regard to understanding pharmaceutical prescriptions and prescription drug labels (Wolf, Davis, Tilson, Bass, \& Parker, 2006), informed consent documentation 
(Donovan-Kicken et al., 2012), the need for and process of obtaining hormone therapy for postmenopausal health (Torres \& Marks, 2009), the consequences of delayed childbearing (Gossett, Nayak, Bhatt, \& Bailey, 2013), and the biological causes of disease symptoms (Olson, Blank, Cardinal, Hopf, \& Chalmers, 1996). Low health literacy is also associated with less certainty in advance care planning treatment preferences (Sudore, Schillinger, Knight, \& Fried, 2010) and less knowledge of heart disease (Ussher et al., 2010) among older adults. As found by Gazmararian, Parker, and Baker (1999), women with low health literacy are less knowledgeable about how to prevent pregnancy and are therefore less likely to correctly use birth control.

It is important to keep in mind that common vocabulary used by physicians may be viewed as jargon to patients. In a study assessing the comprehension of 50 commonly used words in medical interviews, only 35\% of patients understood the word "orally," only 18\% understood “malignant," and only 13\% understood "terminal” (Samora, Saunders, \& Larson, 1961). A more recent study (Davis et al., 2001) on colorectal cancer screening indicated that most patients did not understand words such as "polyp" and "lesion." Studies indicate that individuals with low health literacy have difficulty understanding patient education pamphlets, online information, and instructions from physicians (Williams et al., 2002). These associations are not entirely due to poor general education; van der Heide and colleagues (2013) found that low health literacy mediates the relationship between a low level of education and poor health outcomes.

Poor patient-provider communication. Health literacy has been shown to affect the physician-patient relationship, resulting in less effective communication of health directives (Williams et al., 2002), decreased levels of patient advocacy (Martin et 
al., 2011), decreased perception of patient centered care and lesser quality communication with healthcare organizations (Wynia \& Osborn, 2010), more negative interactions with healthcare providers (Manganello \& Clayman, 2011), and greater likelihood to avoid visits with a physician (Morris et al., 2013). Many patients have difficulty understanding the medical directives they are provided, with some individuals reporting remembering less than $50 \%$ of what was said to them by a physician immediately following the medical interview (Ong, de Haes, Hoos, \& Lammes, 1995; Williams et al., 2002). A meta-analysis conducted by Williams and colleagues (2002) revealed that patients with low health literacy struggle to understand the complex terminology and vocabulary used by physicians, resulting in inadequate and confusing communication between providers and patients. In fact, patients across several studies complained that providers did not explain their illness in terms they could understand.

Issues related to health literacy extend to the communication between nurse practitioners and patients. Cafeiro (2013) found that although nurse practitioners have high intentions to address health literacy in practice, their knowledge of health literacy and actual use of strategies to accommodate low health literacy was low. Specifically, nurse practitioners incorrectly answered questions regarding health literacy's effect on healthcare status, screening tools for low health literacy, and evaluation measure of educational materials. The practice of screening patients for low health literacy was not enforced, and written materials were given to patients without the option of alternate formats such as audiotapes, videotapes, or computer programs (Cafiero, 2013). Relatedly, Dickens, Lambert, Cromwell, and Piano (2013) found that inpatient nurses often overestimate a patient's health literacy; in fact, the ratio of underestimation to 
overestimation was 6 to 1 . As Cafiero (2013) contended, improving nurse practitioners' knowledge of health literacy and health literacy strategies is a very important step toward improving patient education and outcomes. As the nurse is the healthcare professional responsible for patient understanding of information pertinent to follow-up appointments, dietary restrictions, medications, activity level after discharge, and at-home treatments, the communication between patients and nurses is likely a very important factor in the association between low health literacy and the negative outcomes discussed in this section.

Shame, stigma, and denial. A potential major facet of the lack of understanding established in the medical interview when the patient has a low level of health literacy is the shame, stigma, and/or denial experienced by the patient (Parikh, Parker, Nurss, Baker, \& Williams, 1996). Individuals with low health literacy tend to ask fewer questions during the medical interview, are less able to describe their medical condition to the healthcare provider, and (perhaps more dangerously) do not see their literacy level as important to their health outcomes (Parikh et al., 1996; Roter, 2000). As a result, these individuals do not mention their inability to understand medical terminology and directives with their healthcare provider (Roter, 2000). Individuals with low levels of health literacy often do not disclose their inability to read and understand health information to anyone, including family and friends. Parikh and her research team (1996) found that $67.2 \%$ of individuals with low health literacy have never told their spouses about their complications, and 19\% had never disclosed their difficulties to another person. These individuals reported navigating the medical environment by watching others, making excuses, and asking other patients for help. Older adults with 
low health literacy experience increased discomfort regarding asking for further explanations of information related to coronary heart disease, and feel less support with discussing their health problems with family, friends, and healthcare professionals (Ussher et al., 2010). As Parikh et al. (1996) urge, improvements to the healthcare system should include educational interventions to help hospital staff address low health literacy among patients without causing embarrassment or shame or inducing larger cultural stigmas regarding illiteracy.

Poor treatment adherence. There are many studies which provide evidence of an association between low health literacy and decreased compliance with recommended treatments (Williams et al., 2002). In a study examining the association between health literacy and adherence to antiretroviral therapy treatment for HIV, Kalichman and colleagues (2008) found that health literacy predicted an individuals' adherence to the treatment regimen more so than any other factor included in the study, which included emotional distress, internalized stigma, and social support. A related study examining adherence to the antiretroviral treatment regimen, and using pharmacy refills as an objective indicator of adherence, also found an association between health literacy and adherence (Graham, Bennett, Holmes, \& Gross, 2006). Low health literacy has also been associated with low rates of medical compliance to glaucoma therapy (Muir et al., 2006), a triple drug retroviral treatment plan for HIV (Kalichman, Ramachandran, \& Catz, 1999), medication refill adherence in cardiovascular-related disease (Gazmararian et al., 2006), use of complementary and alternative medicine (Gardiner et al., 2013), and medical adherence with a physician’s recommendations more generally (Ngoh, 2009). Although several other factors, such as beliefs and attitudes toward a medical treatment, 
might mediate the relationship between health literacy and adherence (Graham et al., 2006), research indicates that health literacy alone is influential in individual's health management decisions (Kalichman et al., 2008).

Adverse health outcomes. Individuals with low health literacy are more than two times more likely to self-report poor health than those at adequate levels of health literacy (Baker, Parker, Williams, Clark, \& Nurss, 1997; Gazmararian et al., 1999). In a meta analysis of 684 articles on the association between health literacy and poor health outcomes, DeWalt, Berkman, Sheridan, Lohr, and Pignone (2004) found that individuals with low health literacy were 1.5 to 3 times more likely to experience a poor health outcome. These poor outcomes included blood glucose levels (Williams et al., 1998), preteenage alcohol use (Hawthorne, 1996), the presence of hypertension (Battersby et al., 1993), depression (Gazmararian, Baker, Parker, \& Blazer, 2000; Kalichman \& Rompa, 2000), poor vision (Press, Shapiro, Mayo, Meltzer, \& Arora, 2013), reduced executive functioning over time (Sequeira et al., 2013), overall health (Baker et al., 1997; Gazmararian et al., 1999; Sullivan et al., 1995; Weiss et al., 1994). In all cases, lower health literacy was associated with poorer health behaviors and outcomes.

Increased healthcare costs and hospitalization rates. Lack of knowledge and understanding associated with low health literacy leads to increased hospitalization and medical costs (Bernhardt \& Cameron, 2003). Individuals with low health literacy are more likely to be hospitalized (Baker et al., 2002; McArdle, 2000), perhaps as a result of engaging in riskier behaviors and having less understanding of prescription drug instructions (Baker, Parker, Williams, \& Clark, 1998). Baker and colleagues (1998) report that individuals with inadequate healthy literacy have a 52\% higher risk of hospital 
admission than individuals with adequate health literacy levels. The severity of the consequences such as these for low health literate adults has led scholars to conclude “research efforts on the challenge of increasing people’s health literacy levels are not only needed, they are overdue” (Bernhardt \& Cameron, 2003, p. 600). These research efforts are further necessitated by the widespread prevalence of low health literacy in the United States.

\section{The Prevalence of Low Health Literacy}

The issue of low reading literacy is a serious issue facing the United States population, with limited literacy skills affecting 34 to 55 \% of U.S. adults (Kutner, Greenberg, \& Baer, 2005). The first National Assessment of Adult Literacy (NAAL; Kutner, Greenberg, Jin, \& Paulsen, 2006) reported that $14 \%$ of adults in the United States have the lowest levels of prose and document literacy, while $22 \%$ operate at the same level for quantitative literacy. This issue of low literacy is further inflated by the context specific nature of health literacy. According to the NAAL, only $12 \%$ of adults in the United States are estimated to be fully capable of functioning in the healthcare environment (Cameron et al., 2011). Overall, it is estimated that anywhere from 80 to 87 million Americans (out of approximately 316 million) are below basic health literacy levels (Berkman et al., 2011; U.S. Census Bureau, 2014; Vernon et al., 2007).

Williams and colleagues (1995) administered the Test of Functional Health Literacy in Adults (TOFLA) to 2,659 patients in two large hospitals. Their results indicated that 35\% of English speaking participants and 62\% of Spanish speaking participants had inadequate or marginal levels of health literacy. Of all participants combined roughly $60 \%$ could not understand a standard informed consent document, 
$42 \%$ could not comprehend directions for taking medicine on an empty stomach, and 26\% could not understand information regarding scheduling for doctor's appointments (Williams et al., 1995). Similarly, Gazmaranian and colleagues (1999) found that 24\% of English speaking and 34\% of Spanish speaking participants in their sample of 3,260 adult patients had marginal health literacy, and that lower health literacy was positively associated with being older, having completed less school, having a blue collar occupation, having a cognitive impairment, and being a part of a racial or ethnic minority group. In a meta-analysis of 85 studies with 31,129 participants, Paasche-Orlow, Parker, Gazmaranian, Nielsen-Bohlman, and Rudd (2005) found that low health literacy was significantly associated with several demographic variables, including: education level, age, ethnicity, and income. Low health literacy is more prevalent among older adults, individuals belonging to an ethnic minority group, lower income brackets, and those with less than a high school education.

A national survey on health literacy skills (Kutner et al., 2006) revealed that more than one-third of adults in the United States cannot determine the correct way to take prescription medicine based on the instructions provided on the label, while $85 \%$ cannot correctly calculate an employee share of health insurance cost using a table. The researchers concluded that only one in 10 Americans possess the sufficient skills to conduct the wide array of mental and physical tasks required in the nation's current healthcare system. In a nationally representative adult sample gathered by the U.S. Department of Health and Human Services (2013a), only 60.6\% of adults ages 18 and over report that their healthcare provider explained medical terminology in a way that they could understand. These results varied by ethnicity, with $66.5 \%$ of non-Hispanic 
black adults reporting that their healthcare providers explained medical directives in a way they could understand, compared with $60.9 \%$ of non-Hispanic white adults.

Given the substantial percentage of Americans categorized as low health literate, in combination with the influence of literacy on healthcare access, disparities, and healthcare costs, the study of health literacy has emerged as a highly prioritized issue in U.S. policy agenda (McCormack et al., 2013). However, the proliferation of various competing conceptualizations and measurements of the construct has hindered progress in both academic and practical applications. In the following section a review of the current state of health literacy measurement is provided, providing evidence of the potential impact of this dissertation on the study of health literacy.

\section{The Measurement of Health Literacy}

Despite the claims that that research examining the antecedents and outcomes of low health literacy is an issue of national concern (McCormack et al., 2013), a need remains for a measure of health literacy that social scientists can utilize. To date, most measurements of health literacy have been developed for clinical and diagnostic use and are consequently unable to be reliably adapted for use in social science research. As discussed by McCormack and colleagues (2013), the lack of a "gold standard” measurement of health literacy is likely attributable to the number of competing definitions that currently exist for the construct (p. 10). Efforts to develop instruments to assess health literacy, even in the clinical setting, have been given much less attention than efforts to conceptualize the construct and reach a mutually agreed upon definition (Berkman et al., 2010; McCormack et al., 2013). However, the detriment of not having a universally available and reliable means of assessing health literacy has arguably limited 
research on health literacy much more so than its inconsistent conceptualizations (Berkman et al., 2010).

The development of a comprehensive measurement of health literacy may be the most significant and challenging task facing today’s researchers and health practitioners (Pleasant, McKinney, \& Rikard, 2011). There are currently more than 35 published measures of health literacy, yet health communication researchers and practitioners have not yet agreed upon a preferred means of operationalizing the construct (McCormack et al., 2013). These existing measures of health literacy include the Rapid Estimate of Adult Literacy in Medicine (REALM; Davis et al., 1993), the Test of Functional Health Literacy in Adults (TOFHLA; Parker, Baker, Williams, \& Nurss, 1995), the Newest Vital Sign (NVS; Weiss et al., 2005), the Medical Data Interpretation Test (Schwartz, Woloshin, \& Welch, 2005), the Short Assessment of Health Literacy for Spanishspeaking Adults (SAHLSA; Lee, Bender, Ruiz, \& Cho, 2006), the Stieglitz Informal Reading Assessment of Cancer Text (Agre, Steiglitz, \& Milstein, 2006), the Health Literacy Skills Instrument (HLSI; McCormack, et al., 2010), the Health Activities Literacy Scale (HALS; Rudd, Kirsch, \& Yamamoto, 2004), the Measure of Interactive Health Literacy (Rubin, Parmer, Freimuth, Kaley, \& Okundaye, 2011), the Chinese Healthy Literacy Scale for Chronic Care (Leung et al., 2013), and quantitative, computerassisted programs that document the use of complicated medical terminology in the medical interview (Koch-Weser, Rudd, \& DeJong, 2010).

The two most widely used of these measures are the REALM (Davis et al., 1993) and the TOFLA (Parker et al., 1995) (McCormack et al., 2010). The REALM is a word recognition and pronunciation test. The testing procedure involves a hired interviewer 
presenting patients with a sheet of paper containing three lists of 22 words each, arranged according to their number of syllables and pronunciation difficulty. The patient is asked to read as many words aloud as he or she can to the interviewer, beginning with the first word in the first column. If patients are unable to pronounce several consecutive words correctly, the interviewer asks the patient to look down the list of words and pronounce as many of the remaining words as he or she can. Dictionary pronunciation is the scoring standard, and patients are assigned a grade level range (e.g., grade 4-6) based upon their reading ability (Davis, Michielutte, Askov, Williams, \& Weiss, 1998).

The TOFLA uses actual materials a patient may encounter in healthcare setting to determine how well the patient can perform reading and numeracy tests. In taking the assessment, patients are presented with a reading comprehension test that consists of 50 items. The patient is scored based on his or her ability to correctly fill in words that have been systematically deleted from the text. The passages include passages about upper GI series, a Medicaid application, and a procedure consent form. There are also 17 items which address patient numeracy; these assess one’s ability to understand numbers included on prescription bottle labels, blood glucose results, and schedule times on appointment slips (Davis et al., 1998). Both the REALM and the TOFLA are relatively easy to administer, although they require a trained interviewer to administer and score the assessment in person (Yost et al., 2010).

Numerous scholars (e.g., Agre et al., 2006; Baker, 2006; Kalichman \& Rompa, 2000; Lincoln, Arford, Prener, Garverich, \& Koenin, 2013; McCormack et al., 2013; Morrow et al., 2006; Rubin et al., 2011; Schonlau, Martin, Haas, Derose, \& Rudd, 2011; Weiss et al., 2005) have critiqued these scales for a variety of reasons including their 
evaluation of word recognition and not actual comprehension, inability to assess spoken communication skills, inability to distinguish between people of very high and very low health literacy, lack of rigorous psychometric analysis in scale development, use of problematic (re: triggering an emotional response) language, and focus on health literacy as a unidimensional construct (see Pleasant et al., 2011 for a complete list of these critiques). To this point, there are three issues common to these existing health literacy measures that are worthy of note. In the sections that follow, the (a) narrow assessment range, (b) length, and (c) static conceptualization of health literacy characteristic of current assessments will be discussed, followed by an overview on how to move forward in developing an improved scale which addresses these three measurement issues.

\section{Narrow Assessment Range}

As Pleasant et al. (2011) asserted, most existing measures of health literacy only assess a narrow range of reading and numeracy skills, focusing on the competency of the individual without taking into account the more complex situational and contextual constructs (e.g., the competence of the physician) that may account for recall and understanding in the medical interview. As mentioned previously, the two most popular measures of health literacy are the REALM (Davis et al., 1993) and the TOFLA (Parker et al., 1995), both of which assess individual reading capacity. As Baker (2006) observed, neither test is a comprehensive assessment of individuals’ cognitive abilities, but rather each assesses a specific domain of literacy thought to be indicators of overall capacity. The REALM is a 66-item word recognition, vocabulary, and pronunciation test, whereas the TOFLA assesses reading fluency, and consists of a 50-item reading comprehension (re: prose literacy) section and a 17-item section on reading and 
understanding labels and documents commonly used in hospitals (re: numeracy). The REALM and the TOFLA are correlated at .80 , and are also highly correlated with general vocabulary tests (i.e., the Wide Range Achievement Test) (Baker, 2006).

As discussed by Baker (2006), the ideal measure of health literacy would assess individuals' reading fluency, vocabulary, quantitative abilities, and their written and oral communication demands. However, this type of comprehensive assessment would also be impractical for most projects related to health literacy. More comprehensive measures, such as the HALS (Rudd et al., 2004) assess oral, , document, and quantitative literacy across a wider array of dimensions (i.e., health promotion, health protection, disease prevention, healthcare and maintenance, and systems navigation) are not ideal for either researchers nor practitioners, as these tests can take up to one hour to complete (Baker, 2006; Nutbeam, 2008). Instead, the focus should be on developing measures that are short enough for practical use among researchers and practitioners of all levels (Weiss et al., 2005).

\section{Length of Assessment}

The calls for an improved measure of health literacy are often issued with an equally stressed interest in developing a shorter measure of the construct (Baker, 2006). The short version of the TOFLA was developed with this interest in mind, as it takes approximately seven minutes to complete (Baker, Williams, Parker, Gazmararian, \& Nurss, 1999), as was the 8-item (REALM-R, Bass, Wilson, \& Griffith, 2003) and 7-item (Arozullah et al., 2007) revised versions of the REALM. The original version of the TOFLA takes an average of 22 minutes to complete; the original 66-item REALM takes approximately three minutes to complete (Davis et al., 1998). The Newest Vital Sign 
(NVS; Weiss et al., 2005) was developed to assess a patient's health in much less time than previous measures (i.e., three minutes). In administering the measure, a patient is given a specially designed ice cream nutrition label to review and is asked a series of questions that require reading and numerical ability. Based on the number of correct answers a patient provides to answers regarding ingredients and nutritional content, healthcare providers are then able to assess his or her health literacy level as high, moderate, or low. Weiss et al. (2005) argued that a patient's ability to read and analyze a nutrition label requires the same analytical and conceptual skills needed to understand and follow a healthcare provider's medical directions. The NVS was designed to assess prose literacy, numeracy, and document literacy, and has been validated against dominant measures of health literacy (i.e., the TOFLA; Weiss et al., 2005). Further, Weiss et al. (2005) posited that the NVS is able to distinguish among marginal levels of health literacy more precisely than other assessments given its scoring system.

However, the NVS is undoubtedly best suited for clinical use, as are the STOFLA (Baker et al., 1999) and the 8-item version of the REALM (Bass et al., 2003), and not social science research given the nature of their administration and scoring. Further, these measures are still not sufficient comprehensive measures of health literacy and do not utilize the more complex interaction-based or critical definitions (Nutbeam, 2000) of the construct. In fact, the intended use of many of the measures of health literacy mentioned above is evidenced in the fact that they are not widely available; rather, as Davis and colleagues (1998) report, the tests must be ordered can cost up to \$95 to use (e.g., the WRAT-R-3). In order to provide a point of comparison for the measure to be developed in this dissertation, four of the most commonly used measures are 
provided in Appendices A-D. These are the REALM, the REALM-SF, the STOFLA, and the NVS. The third and final issue to be discussed regarding existing measures of health literacy concerns this lack of depth, as the static or fixed conceptualization of health literacy used in these measures is limiting in terms of both research and practice.

\section{Static Conceptualization of Health Literacy}

In addition to the difficulty of administering these measures, many of the aforementioned scales both conceptualize and operationalize health literacy as a fixed characteristic of an individual. In doing so, these measurements suggest that health literacy is a stable attribute of an individual that only needs to be measured once. The research and practical application based upon these measures therefore are based on the assumption that health literacy is relatively constant throughout a person’s life and should be accommodated, not increased (Berkman et al., 2010).

There is a need for a scale that conceptualizes health literacy as an asset that can be developed and improved over time through education and communication (Berkman et al., 2010). In conceptualizing health literacy as dynamic in this way, the implication is that a person's health literacy can be altered by various health circumstances and experiences. In contrast to the fixed or static conceptualization of health literacy, a dynamic approach also requires health literacy to be measured repeatedly and periodically over time (Berkman et al., 2010). The move toward conceptualizing health literacy as dynamic is inevitable with increased sophistication in both measurement and application in the study of the construct (Berkman et al., 2010). Importantly, the conceptualization of health literacy as dynamic promotes the development and implementation of educational efforts and communication programs meant to increase 
health literacy as a means to enabling individuals to exert greater control over their health (Nutbeam, 2008).

If health literacy is a fixed or static characteristic of an individual, it can at best be accommodated by healthcare professionals and others interacting with a low health literate person. If health literacy is dynamic, an individual can take efforts to improve the skills he or she needs to function effectively in the healthcare environment. In conceptualizing and operationalizing health literacy as a dynamic construct, this dissertation places an emphasis on developing ways to improve upon individuals' health literacy levels.

The most notable facet of the calls for further development of health literacy measurements are the repeated requests for assessments of individual's social and/or communicative skills. Baker (2006) called for improved self-report measures which are able to tap into individuals’ perceptions of their own understanding of spoken instructions in the health context. Baker argued that such an assessment would directly assess the mismatches in individual capacity and communication demands, and provide important information from the patient's perspective. Importantly, Baker specifically called on communication studies scholars to develop this measure due to their expertise in assessing the comprehension of oral speech, In a study by Wallace, Rogers, Roskos, Holiday, and Weiss (2006), the question "How confident are you in filling out medical forms by yourself?” had the best predictive value for identifying individuals in a primary healthcare clinic with a low REALM score, over and above the influence of any demographic variables. As Baker (2006) noted, there has yet to be an established selfreport assessment of one's comprehension of spoken health-related information, and it 
appears to be a sorely needed instrument in the search to better understand and predict health literacy and its outcomes.

There is still much work to be done in the way of developing a measurement that will assess individual health literacy in the way of a person's ability to gain access to, understand, and use health information as a means of promoting and maintain good health (i.e., the WHO definition of the construct; Nutbeam, 2008; WHO, 2014). In their call for improved measurement of health literacy, Pleasant et al. (2011) highlighted the need for prioritization of social science research in these efforts, so that more complex and comprehensive advancements can be made toward addressing the issue of health literacy. Solely diagnosing individuals as low health literate in clinical practice is not a sufficient means of handling the outcomes associated with this phenomenon; health literacy needs to be addressed in research on health communication and public health (Johnson, Baur, \& Meissner, 2011; Pleasant et al., 2011).

\section{Problem Statement}

Parker, Ratzan, and Lurie (2003) urged researchers to conduct further investigations into the construct of health literacy, to offer strategies to create a healthliterate America, and to influence policy changes that would help ameliorate the problem of low health literacy. Their suggestions included conducting multidisciplinary research on health literacy and its measurement, engaging the federal government by making it a priority for research funding, and improving medical practice by including health literacy in training medical professionals. In 2010, Parker and Ratzan updated this work on a commentary published in the Journal of Health Communication, and noted that although 
significant strides have been made in the way of understanding health literacy, much still remains to be done:

Problems with health literacy are real, growing, and here to stay. The last decade was spent cataloging the problem, defining the issue and promoting health literacy as essential to decreasing costs, reducing disparities and improving quality of care... Now, we must continue to build an effective healthcare system that embraces the value of the ability to effectively communicate what works (Parker \& Ratzan, 2010, p. 30). There are a number of aspects of the low health literacy epidemic that require more attention in the health communication literature. Parker and Ratzan (2010) contended that understanding and embracing quantifiable outcomes related to health literacy is crucial to the long-term success of efforts to improve health literacy in America. The authors provide six recommendations for future work on health literacy, including (a) recognizing that health literacy is foundational to reforming health and healthcare in the United States, (b) developing and advancing metrics for measuring progress in improving health literacy, (c) integrating lessons of the growing body of evidence on health literacy into coordinated national efforts to improve patient safety, preparedness, and response, (d) supporting health literacy research, its dissemination, and implementation, (e) advancing new media opportunities to communicate health information that is understandable and navigable, and (f) the commission of a follow up Institute of Medicine report on health literacy.

There is currently not a strong focus on enhancing individuals’ level of health literacy. Rather than accommodating to a stable level of health literacy, health 
communication researchers and educators should focus on the dynamic nature of health literacy and how to increase individuals' cognitive and social skills needed to improve functioning in the healthcare environment and health outcomes (Nutbeam, 2000). Adding to this problem, research concerning health literacy has been almost entirely devoid of theory. Although several major theories of health communication address the importance of self efficacy and response efficacy (e.g., TTM; Prochaska \& DiClemente, 1983; HBM; Rosenstock, 1966; EPPM; Witte, 1992), these theories have not been employed to their full potential to either inform how health literacy functions in contexts beyond the medical interview, or to develop effective interventions.

One possible explanation for the general lack of theory development in the study of health literacy is due to poor measurement. Existing measures of health literacy are largely developed for clinical use, and do not provide an assessment of the oral and selfreported skills that individuals possess regarding their ability to obtain, understand, and apply health information in their everyday lives (Pleasant et al., 2011). By increasing the precision in the conceptualization and operationalization of health literacy, researchers can begin to make advancements in understanding the complexity of this construct (Johnson et al., 2011). Cameron and colleagues (2011) reinforce the significant of health literacy:

Health literacy is an integral part of the study of health communication. If people are unable to access, understand, and apply the health-related information they receive, then whether or not the "best" message is reaching the population is no longer an important question. The question 
becomes much more basic: whether or not a message, any message, is reaching the population. (p. 316).

As both Cameron and colleagues (2011) and Ratzan (2011) asserted, there is a critical need to incorporate health literacy into current academic research and practical application in health communication. For instance, McCormack and colleagues (2013) discuss the various models that might arise from an improved measure of health literacy: the examination of factors that mediate the link between health literacy and health outcomes might include studies on health status, attitude, motivation, self-efficacy, emotions, culture, socioeconomic status, and other ecological factors. These models could incorporate health system moderators at both individual and system level analysis (McCormack et al., 2013). McCormack and colleagues (2013) contend that "to effectively monitor health literacy over time, examine its relationships with key variables, and promote stability across studies, more widely accepted measures... are needed" (p. 13). Developing a conceptual model of health literacy which accounts for individual-, group-, and population-level characteristics would add both clarity and rigor to a rapidly growing area of study in health communication (McCormack et al., 2013).

Although healthcare practitioners and health educators have realized the importance of this construct in their line of work with increasing emphasis in the past 10 years, health communication scholars have yet to fully contribute to the discussion of how to help over half of the United States population to achieve optimal health outcomes. In their call for increased efforts toward conducting 
basic research on health literacy, Johnson and colleagues (2011) argue that health literacy researchers are "eager to move beyond identifying the problem in order to design interventions to solve it” (p. 23). Understanding the basic mechanisms that create and perpetuate low health literacy is a crucial first step in developing efficient and effective interventions (Johnson et al., 2011).

This dissertation seeks to contribute to the study of health literacy in the field of Communication Studies by addressing two specific purposes. First, a valid and reliable measurement of health literacy appropriate for use in social science will be developed. This measurement of health literacy will be based upon the World Health Organization’s (WHO; 2014) definition of the construct, thus promoting a focus on both the cognitive and social aspects of an individual's health literacy level.

Second, this dissertation seeks to determine whether health literacy can be increased through communication interventions. The definition of health literacy used to guide this research focuses on "the cognitive and social skills which determine the motivation and ability of individuals to gain access to, understand and use information in ways which promote and maintain good health” (WHO, “Track 2: Health literacy and health behavior,” para. 1, 2014). If health literacy is a set of skills, then effective messages should work in increasing the health literacy levels of individuals exposed to these messages. Further, if the measure developed for the first purpose of the study is an accurate reflection of the WHO definition of the construct, it should be able to assess changes in health literacy due to these interventional efforts. 
The WHO definition of health literacy is important in its emphasis on the notion that "health literacy means more than being able to read pamphlets and successfully make appointments," noting that "by improving people’s access to health information and their capacity to use it effectively, health literacy is crucial to empowerment” (WHO, 2014, Track 2: Health literacy and health behavior,” para. 1). A crucial aspect of the WHO's initiative in their global address on health promotion involves exploring how people can develop the skills, knowledge, and efficacy to act on that knowledge to promote good health (WHO; 2014), thus promoting the idea that health literacy can, and should be, influenced and improved through health education and participation programs. In the Healthy People 2020 objectives for health communication and health information technology (U.S. Department of Health and Human Services, 2013b), increasing health literacy and building health knowledge and skills are proposed as goals for healthcare practitioners, researchers, and other health professionals to strive for in the next ten years. Finding ways to improve public education regarding health information (and therefore increasing health literacy levels) is a public health priority (Paasche-Orlow \& Wolf, 2010).

The two major goals of this manuscript are to (a) develop a valid and reliable measurement of health literacy based on the WHO (2014) definition of the construct and (b) to assess whether health literacy is a skill set that can be increased through communication interventions. To achieve these, the set of research questions and hypotheses outlined in the next section are proposed. 


\section{Rationale}

Employing the WHO (2014) definition of health literacy as a conceptual basis, LaBelle and Weber (2013) sought to develop a measure of oral health literacy in line with the demands of social science research (Pleasant et al., 2011). Specifically, the researchers aimed to assess individuals' perception of their own health literacy in communicative interactions with their healthcare provider. Drawing from research on health literacy, the initial pool of twenty items shown in Table 1 was designed to reflect an individual's ability to gain access to, understand, and use health information following an interaction with a healthcare provider (WHO, 2007; 2013a).

After conducting an exploratory factor analysis on the measure, LaBelle and Weber (2013) concluded that the best solution was a 10-item measure with three factors. The first factor, consisting of five items, was named Question Asking as it concerned participants' perceived ability and behaviors related to asking questions of a healthcare provider (e.g., "If I do not understand a term that my healthcare provider has used, I do not hesitate to ask him or her to clarify"). The second factor, consisting of three items, was named Adequacy of Information as it concerned participants' perceptions of feeling satisfied with the amount of information or length of time they received with a healthcare provider (e.g., "I often feel my time with my healthcare provider was not long enough”). The third factor, consisting of two items, was named Ability to Apply Health Directives as it concerned participants' perceptions that they could recall and apply what they had learned from their healthcare provider (e.g., "I feel confident that I am able to apply the medical directives given to me by my healthcare provider when I leave his or her 
Table 1.

Original Item Pool for Perceived Oral Health Literacy Scale

Items

1. I feel confident that I am able to apply the medical directives given to me by my healthcare provider when I leave his or her office.

2. I confident in my ability to ask questions of my healthcare provider in conversation with him or her.

3. I am able to understand the directions my healthcare provider gives me.

4. When I leave an interaction with my healthcare provider, I am confident that I recall the key points of his or her message.

5. I am motivated to understand the directions given to me by my healthcare provider.

6. I directly ask questions of my healthcare provider.

7. When I have a question for my healthcare provider, I make sure I ask it.

8. I worry that my healthcare provider will think my questions are "dumb."

9. When my healthcare provider is talking to me, I am able to understand most of what he or she is saying.

10. When I leave my healthcare provider's office, I remember exactly what he or she told me to do.

11. I am confident in my ability to listen to my healthcare provider when he or she is talking.

12. I fully listen to my healthcare provider when he or she is talking.

13. When I ask my healthcare provider a question, he or she fully listens to me.

14. I often leave interactions with my healthcare provider feeling confused.

15. When I leave interactions with my healthcare provider, I feel like I have said everything that I wanted to say.

16. I understand the terms my healthcare provider uses in interactions with me.

17. If I do not understand a term that my healthcare provider has used, I do not 
hesitate to ask him or her to clarify.

18. I often feel my time with my healthcare provider was not long enough.

19. I leave interactions with my healthcare provider feeling very informed about my health.

20. I am motivated to listen to my healthcare provider when he or she is talking to me. 
Table 2.

EFA Factor Loadings for Perceived Oral Health Literacy Scale

\begin{tabular}{lllll}
\hline Items & Factor 1 & Factor 2 & Factor 3 & $\mathrm{M}, \mathrm{SD}$ \\
\hline
\end{tabular}

1. I feel confident that I am able to apply the .13

.71

.16

$4.05, .84$

medical directives given to me by my healthcare provider when I leave his or her office.

2. I am confident in my ability to ask questions of .09

$\begin{array}{lll}.82 & .04 & 4.00, .86\end{array}$
my healthcare provider in conversation with him or her.

3. When I leave an interaction with my healthcare provider, I am confident that I recall $\begin{array}{llll}.31 & .16 & .54 & 4.13, .70\end{array}$ the key points of his or her message.

4. I directly ask questions of my healthcare provider.

$\begin{array}{llll}.66 & .25 & .27 & 3.86, .93\end{array}$

5. When I have a question for my healthcare .20

.13

.90

3.94, .94 provider, I make sure I ask it.

$\begin{array}{llll}.87 & .03 & .16 & 3.62, .88\end{array}$
provider feeling confused.

7. When I leave interactions with my healthcare provider, I feel like I have said everything that I wanted to say.

8. If I do not understand a term that my healthcare provider has used, I do not hesitate to ask him or her to clarify.

9. I often feel my time with my healthcare provider was not long enough.

$\begin{array}{llll}.73 & .13 & .32 & 3.81, .98\end{array}$

10. I leave interactions with my healthcare provider feeling very informed about my health.

$\begin{array}{llll}.60 & .06 & .18 & 3.96, .92\end{array}$

.29

.50

.39

3.42, 1.1 
office”). The items and factor loadings of the factor analysis are presented in Table 2.

The scale instructions and response categories for the Perceived Oral Health Literacy Scale are presented in Appendix E.

LaBelle and Weber (2013) were able to demonstrate the reliability and construct validity of this measure by assessing its relationship to individuals' assessments of perceived health competence (Smith, Wallston, \& Smith, 1995). Individuals' scores on the POHLS were significantly and positively $(r=.52, p<.001)$ related to scores on the Perceived Health Competence Scale (Smith et al., 1995). Notably, the correlation between these two constructs did not exceed .70, at which point researchers should be concerned that the measures are isomorphic and are not reflecting distinct constructs (Dembrowski, 1968; Weber \& Patterson, 2000).

Although the development of the Perceived Oral Health Literacy Scale (POHLS) was an important step toward providing an assessment of health literacy to be used in social science research, it was a preliminary effort in need of further revision. Specifically, the factors that emerged from the data (i.e., Question Asking, Adequacy of Information, and Ability to Apply Medical Directives) were based upon participants' reflections upon their last interaction with their own healthcare provider. Therefore a number of extraneous factors may have been affecting participants' responses to the original item pool, including hindsight bias, difficulties in accurately recalling the interaction, and general satisfaction with the healthcare provider (LaBelle \& Weber, 2013). It is entirely likely that the quality of one's healthcare provider, and not his or her own health literacy, could have driven these factors to emerge. LaBelle and Weber (2013) state that the items 
designed in their study were "created to measure individuals' perceived ability to understand their health provider, listen fully to him or her during the medical interview, and ask questions as needed" (p. 8). This is admittedly more of a function of the quality of communication between a healthcare provider and patient and less so of the patients' motivation and ability to manipulate health information correctly. As health literacy encompasses a much wider set of communicative events than simply interactions with healthcare providers (Baker, 2006; Nutbeam, 2000), it is important for the measurement to reflect how individuals gain access to, understand, and use health information across varied contexts.

Additionally, the items developed for the Perceived Oral Health Literacy Scale (LaBelle \& Weber, 2013) were based upon individuals’ perception of their own abilities (e.g., "I feel confident that I am able to apply the medical directives given to me by my healthcare provider when I leave his or her office"). The items developed did not operationalize an individual's motivation to gain access to, understand, and use health information, which is a critical aspect of the WHO (2014) definition of the construct.

LaBelle and Weber (2013) argued that additional research should be conducted to further validate the factor structure and dimensionality of this measure and to establish the validity of this POHLS measure. The first research question of this dissertation aims to further refine the POHLS by creating items which reflect more of the complex dimensionality of one's motivation and ability 
to gain access to, understand, and use health information per the WHO definition of health literacy (WHO, 2014).

RQ 1: How can the POHLS be refined to more accurately represent an individual's motivation and ability to gain access to, understand, and use health information?

The revised POHLS will be subjected to further tests to assess whether or not it is actually assessing what it purports to measure, known as validity (Kerlinger, 1986). In line with McCormack and colleagues’ (2013) recommendations, this dissertation seeks to establish multiple forms of validity for the new measure of health literacy put forth in it. Specifically, the content, construct, known groups, and predictive validity of the revised POHLS will be assessed.

\section{Content Validity}

Content validity refers to the representativeness of sampling adequacy of the content of a measuring instrument (Kerlinger, 1986). In the case of self-report measures, the content validity of the assessment therefore refers to the degree to which individual items represent the full range of the construct being measured (Field, 2009). The content validity of a measure is established by showing that the test items are a sample of the domain the researcher is seeking to measure (Cronbach \& Meehl, 1955). As Kerlinger (1986) stated, the driving question behind an assessment of content validity is whether the content of the measure is representative of the universe of content it is meant to assess. The universe of this content, then, must be clearly defined before attempting to determine this type of 
validity (Kerlinger, 1986). The revised POHLS will therefore be gauged for its representativeness of individuals' motivation and ability to gain access to, understand, and use health information, per the definition of the construct that is being employed in this study (WHO; 2014). Thus, the first hypothesis:

H 1: The measurement model of the revised POHLS will provide a good fit to the data.

\section{Construct Validity}

In addition to establishing the content validity of the POHLS, this dissertation also seeks to establish the convergent validity of the POHLS by establishing its relationship with theoretically related variables or constructs (Campbell \& Fiske, 1959; Cronbach \& Meehl, 1955; Field, 2009). As discussed by Kerlinger (1986), construct validity concerns the theoretical relationships one’s measure has with other variables. One accepted way to establish the construct validity of a scale, then, is to assess its relationship with other constructs to which it should be related (Singleton, Straits, \& Straits, 1993; Weber, Martin, \& Cayanus, 2005). Notably, construct validity consists of both convergence and discriminability (DeVellis, 2012; Kerlinger, 1986). Convergence or convergent validity refers to the relationship of a measure or construct with similar constructs; discriminability or discriminant validity means that "one can empirically differentiate the construct from other constructs that may be similar, and that one can point out what is unrelated to the construct" (Kerlinger, 1986, p. 421). In regard to the measure being assessed in this study, the revised POHLS should be related to constructs that are theoretically similar, but should not be 
isomorphic with those constructs or related to constructs that do not make sense theoretically.

In this dissertation, the construct (convergent and discriminant) validity of the revised POHLS will be assessed by determining its relationship to individuals' levels of perceived health competence, patient efficacy, and communication anxiety.

Perceived health competence. One potential construct that should be related to oral health literacy is one's perceived health competence, or the degree to which an individual feels capable of effectively managing his or her health outcomes (Smith et al., 1995). Perceived health competence has been shown to be positively related to an individual's overall physical and psychological wellbeing and health, sense of control over health outcomes, and both intention and enactment of positive health behaviors (Smith et al., 1995). Perceived health competence has been shown to be related to perceptions of self-efficacy and optimism among patients recovering from orthopedic surgery (Waldrop, Lightsey, Ethington, Woemmel, \& Coke, 2001).

Theoretically, an individual's perception of health competence should be related to a sense of understanding and confidence in medical interactions. Low health literacy is related to a variety of negative medical outcomes, including decreased patient satisfaction after the medical interview (Berkman et al., 2011); low perceived health competence is related to lowered physical and psychological well-being (Smith et al., 1995). Given that health literacy and health competence lead to similar outcomes in patient-physician interactions, it should follow that an 
individual's perception of his or her health competence is related to the ability to engage in question asking, understanding and application of medical directives.

However, as stated above, in order to establish construct validity not only does a measure need to be associated with other theoretically related variables (i.e., convergence), but it must also be able to differentiate itself from other constructs (i.e., discriminability). In other words, in the discussion of discriminability, it must be established that any new measure of a construct needs to show that it is distinct from measures of similar and related constructs (Kerlinger, 1986). As such, in order to argue for the validity of the newly developed measure of perceived oral health literacy, it needs to be distinct from the existing measure of perceived health competence (i.e., that the two measures are not assessing the same construct). One way that the construct validity of the revised POHLS may be illustrated is by examining its relation to a measure of perceived health competence. Specifically, the resulting correlation values should indicate a high overlap in variance between one's health literacy and his or her perceived health competence, but these correlation values should not exceed a level (i.e., .70) that indicates the constructs are not accounting for unique variance (Dembrowski, 1968). In order to assist in establishing the construct validity of the scale, the following hypothesis is forwarded:

H 2: Health literacy will be positively correlated to perceived health competence at a level no higher than .70.

Patient efficacy. The second construct utilized in this study in order to determine the construct validity of the revised POHLS is patient efficacy. An 
individual's perception of efficacy across various health situations is thought to be comprised of both self and response efficacy (Witte, 1992). Perceived selfefficacy comprises the individual's belief in his or her ability to perform the recommended response. Perceived response efficacy is the individual's degree of confidence that the recommended response will be effective (Witte, 1992). As discussed by Witte (1992), if either self or response efficacy is low, the individual will be discouraged from enacting the recommended behavior.

Based on the work of Bandura (1977), efficacy is regarded as a crucial component of an individual's success in a wide variety of health communication contexts (e.g., Hale \& Trumbetta, 1996; Marks \& Allegrante, 2005; Witte, 1992). An individual's perception of his or her own efficacy is positively related to his or her participation in the medical interview with a physician (Young \& Klingle, 1996), success in recuperating from a medical trauma such as a stroke (RobinsonSmith \& Pizzi, 2003), self- management of chronic disease (Bodenheimer, Lorig, Holman, \& Grumbach, 2002; Holman \& Lorig, 2004), and behavior change across a wide variety of health behaviors including but not limited to smoking, contraception, weight management, alcohol abuse, and exercise behaviors (see Strecher, DeVellis, Becker, \& Rosenstock, 1986).

In discussing the importance of examining patient participation, efficacy, and other individual factors that impact the physician-patient interaction, Young and Klingle (1996) echo much of the rationale for understanding the epidemic of low health literacy. As the researchers state: 
Although patient participation is acclaimed to be a much healthier model of the doctor-patient encounter than the traditional paternalistic paradigm, there has been a dearth of research that has empirically assessed the effects of patient involvement or addressed barriers that must be overcome before patients can actively participate in medical encounters (p. 29).

Given that both of these constructs concern the quality of communication between physicians and patients, and both have a documented impact on the patient-provider relationship, as well as actual health outcomes for patients (Berkman et al., 2011; Marks \& Allegrante, 2005), it should follow that an individuals' level of health literacy is related to his or her own perception of efficacy in the medical encounter.

It is equally important to establish that a measure of health literacy does not overlap with perceptions of efficacy in the health context. Health literacy represents a distinct set of social and cognitive skills that should be related to but not isomorphic to individuals' perceptions of self and response efficacy as a patient. In a study on informed consent forms conducted by Donovan-Kicken and colleagues (2012), evidence was provided that self efficacy acted as mediator between health literacy and individuals' sense of understanding regarding informed consent procedures. It should then follow that the new measure of health literacy in this dissertation is related to, but not isomorphic with, individuals' perceptions of their own efficacy as a patient. The third hypothesis is proposed: 
H 3: Health literacy will be positively correlated to patient efficacy at a level no higher than .70.

Communication anxiety. The third construct that will be utilized in this study to assess the construct validity of the revised POHLS is communication anxiety. Communication anxiety or avoidance is defined as “an individual's level of fear or anxiety associated with either real or anticipated communication with another person or persons” (McCroskey, 1977, p. 78). This fear or anxiety is influenced by individual characteristics, situation factors, or their interaction (Booth-Butterfield, 2008). Trait communication anxiety is reflected in enduring, cross-situational fear of communication with others; State communication anxiety occurs in a specific situation, and is "the hereand-now response of a person to some situation” (Booth-Butterfield \& Gould, 1986, p. 195). One of the most studied concepts in the field of Communication Studies (Allen \& Bourhis, 1996; Booth-Butterfield, 2008; McCroskey, 1977; McCroskey, 2008; McCroskey \& Beatty, 1986), communication anxiety is associated with a number of negative outcomes including avoidance of communication encounters, withdrawal or minimization of interactions that cannot be avoided, cognitive interference which leads to a disruption of information processing and behavior (Booth-Butterfield, 2008; BoothButterfield \& Booth-Butterfield, 1986; McCroskey, 1977; McCroskey \& Beatty, 1998). Further, the effects of high communication anxiety have been examined across a number of contexts, including in health-related environments (Booth-Butterfield, 2008). Increased levels of communication apprehension have been negatively associated with willingness to discuss gynecological health topics with a physician (Wheeless, 1984), lower satisfaction in communicating with a physician (Ayres, Colby- Rotell, Wadleigh, \& 
Hopf, 1996; Richmond, Smith, Heisel, \& McCroskey, 1998), and lower satisfaction with the quality of medical care (Richmond et al., 1998). Given the association of communication anxiety and apprehension with health outcomes that have demonstrated a relationship with health literacy (Bernhardt \& Cameron, 2003; Williams et al., 2002), it theoretically follows that an individual's fear of communication should be related to his or her health literacy. Notably, however, the communication anxiety examined in this study is not context bound to the physician-patient relationship, as the effects of health literacy occur across contexts. In this study, an individual's trait communication anxiety will be examined in addition to his or her apprehension regarding communication in three contexts: interpersonal or dyadic interactions, meeting or small group interaction, and public speaking contexts (Booth-Butterfield \& Gould, 1986). In sum, the fourth hypothesis is forwarded:

H 4: Health literacy will be negatively correlated with communication anxiety at a level no higher than .70.

\section{Known-Groups Validity}

In establishing the known-groups validity of a measure, a researcher is attempting to demonstrate that the measure can differentiate one group from another (DeVellis, 2012; Eagly \& Chaiken, 1993; Robinson, Shaver, \& Wrightsman, 1991). The purpose behind this test might be theoretical (e.g., using a scale on attitudes toward a certain group to differentiate which members are a part of the group and who is not) or predictive (e.g., using a series of measures to predict employee turnover; DeVellis, 2012). When the measure is used in the former manner, theoretically, a construct validity test is being conducted; when 
the test is purely predictive, a criterion-related validity test is being conducted (DeVellis, 2012). The use of known-groups validation to assess the construct validity of a scale is not uncommon in social science research (e.g., Floyd \& Morman, 1998; Langevin, 2009). Portney and Watkins (2000) discuss knowngroups validity in the following manner:

The most general types of evidence in support of construct validity is provided when a test can discriminate between individuals who are known to have the trait and those who do not. Using the known groups method, a criterion is chosen that can identify the presence or absence of a particular characteristic, and the theoretical context behind the construct is used to predict how different groups are supposed to behave. Therefore, the validity of a particular test is supported if the test's results document these known differences. (p. 89).

As Sharf (1999) noted, the growth of interest in health communication within the larger discipline of communication studies has been rapid and continually expanding. This growth has included an exponential increase in the number of departments that have added courses in Health Communication to their course offerings, as well as in student interest in careers related to health information and delivery (Sharf, 1999). The increases in enrollment and interest in these courses is not only based upon their relevance and utility to success in healthcare, but also due to students' own personal experiences and subsequent interest in the healthcare system (Sharf, 1999). Further, students value courses in which the material can be practically applied to their own lives and help them in 
their future careers (Rice, Stewart, \& Hujber, 2000). Therefore, it should follow that students who complete a course in Health Communication should, by the end of the semester, have increased cognitive and social skills in their ability to apply, use, and understand health information (World Health Organization, WHO, 2014), particularly over students who have not participated in such a course. Given these assumed theoretical differences in the skill set of students who have completed a course in Health Communication and students who have not, the fifth hypothesis is posed:

H 5: University students who have completed a course in Health Communication will have higher health literacy levels than students who have not completed a course in Health Communication.

\section{Predictive Validity}

Criterion-related validity is studied by comparing scores on a scale to external variables which are known or believed to measure the same attribute under study (Kerlinger, 1986). One means of assessing this criterion-related validity is through prediction, although as Kerlinger (1986) notes, prediction in this context need not reference the future. Rather, prediction, or predictive validity, can be established by illustrating how a measure can predict an outside criterion and by checking the relationship of the measurement to some outcome either in the present or in the future. For instance, tests of one's intelligence indicate predictive validity when the tests are associated with current and future ability to succeed in problem solving and learning situations (Kerlinger). Thus, as 
Devellis (2012) contended, criterion-related (specifically predictive) validity is more of a practical concern than a theoretical one. Researchers assessing the predictive validity of a measure are more interested in prediction than understanding the process of how the construct functions (as in construct validity; DeVellis, 2012).

As Kerlinger (1986) asserted, the most difficult aspect of establishing the criterion-related validity of a measure is in selecting the criterion to be used. However, in developing a measure of health literacy, one is guided by research and theory in health communication. As health literacy is related to and affects numerous health outcomes, it theoretically follows that the criteria used to validate its measurement should be derived from constructs important to health communication (Baker, 2006; Cameron et al., 2011; Parker \& Ratzan, 2010). Therefore, to assess the predictive validity of the revised POHLS, this study will utilize two approaches. First, it will be determined if an individual's score on the measure can predict objective indicators of physiological health. Second, it will be determined if an individual's score on the measure can predict his or her placement in the different categories of readiness for behavioral change forwarded by the Transtheoretical Model.

Physiological indicators of health. As defined by the Center for Disease Control and Prevention (CDC), obesity constitutes having a Body Mass Index (BMI) or 30 or higher; one's BMI is calculated as weight in kilograms divided by height in meters squared, rounded to one decimal place (Ogden, Carroll, Kit, \& Flegal, 2012). Nationally, the prevalence of obesity steadily increased during the 
last few decades of the $20^{\text {th }}$ century, and has only recently begun to decrease, or at least level off (Ogden et al., 2012). However, the issue of obesity continues to be a major health issue in the United States, especially given its association with a variety of health problems including cardiovascular diseases (e.g., heart disease and stroke; the leading causes of death in 2008 worldwide; WHO, 2013b), hypertension, musculoskeletal disorders, adverse lipid concentrations, some cancers (e.g., breast, colon, and endometrial), and Type II diabetes (U.S. Department of Health and Human Services, 1998; WHO, 2013b).

According to the National Health and Nutrition Examination Survey, more than one third of adults and almost 17\% of youth in the United States were obese in 2009-2010. Given the risks associated with obesity and the prevalence of this weight status in the United States population, the Healthy People 2020 report includes a number of objectives for healthcare professionals. These include increasing efforts toward improving individual behaviors that lead to obesity as well as addressing the policies and environments that support these behaviors in settings such as schools, worksites, healthcare organizations, and communities (U.S. Department of Health and Human Services, 2013c).

A particularly important health outcome associated with obesity is the development of Type II diabetes (U.S. Department of Health and Human Services, 1998; 2013c). In 2011, the CDC reported that diabetes affects an estimated 25.8 million people in the United States, with as many as 7 million individuals included in that estimate remaining undiagnosed. The consequences of diabetes are severe. Diabetes is the leading cause of kidney failure, 
nontraumatic lower-limb amputations, and new cases of blindness among adults in the United States, is a major cause of heart disease and stroke, and is the seventh leading cause of death in the country (CDC, 2011a). The Healthy People 2020 report also devotes a significant amount of attention to goals and objectives surrounding diabetes, with a particular emphasis on preventing the development of diabetes and improving the quality of care and treatment for those who do have the disease. Overarching issues related to the diabetes epidemic in the nation include, but are not limited to, prevention, testing and early diagnosis, access to care for all persons with diabetes, and improved quality of care overall (U.S. Department of Health and Human Services, 2013d). Many of the objectives put forth in the Healthy People 2020 report, including increased quality of care and self management of both weight status and diabetes, could be informed by research on health literacy. A goal of this dissertation is to investigate the overlap in issues surrounding low health literacy, obesity, and Type II diabetes in the United States from a health communication standpoint.

As stated above, the central concern in establishing the predictive validity of a research instrument is in its relationship to key outcomes of interest to the researcher (Kerlinger, 1986). Therefore, predictive validity is of special concern to applied and practical research endeavors. In order for health literacy to fully reach its potential as a construct employed in health communication research, it must begin to be used in ways that illustrate its utility in not only theoretical development but practical application (Cameron et al., 2011; Parker \& Ratzan, 2010). Given the focus of this study on obesity and diabetes as a health context, 
the predictive validity of the revised POHLS will be determined via the following hypothesis:

H 6: Health literacy will be negatively correlated with physiological indicators of risk for diabetes and obesity.

The transtheoretical model. The Transtheoretical Model was developed by Prochaska and DiClemente $(1982 ; 1983)$ largely in response to an overabundance of theories in health communication and persuasion that were complementary (Slater, 1999). Incorporating theories such as the Elaboration Likelihood Model (Petty \& Cacioppo, 1986), the Theory of Reasoned Action (Fishbein \& Ajzen, 1975), and agenda setting theories in media communication (McCombs \& Shaw, 1972), the Transtheoretical Model offers a comprehensive overview of the foci and boundary conditions of these theories in order to approach a variety of communication problems (Slater, 1999). In fact, the Transtheoretical Model offers a useful framework from which one can describe various types of behavior change, issues with campaign development and implementation, and audiences for which the theory should be most appropriately applied (Slater, 1999).

The Transtheoretical Model (TTM), often referred to as Stages of Change, was developed as a means of understanding behavior change in the context of smoking (Prochaska \& DiClemente, 1983; Slater, 1999). Subsequent research has illustrated its utility with other addictive health behaviors such as alcoholism (Velasquez, Carbonari, \& DiClemente, 1999) and substance abuse (Callaghan et al., 2005). The model has also been used to understand processes of change in 
more general health contexts such as condom use (Naar-King et al., 2006;

Redding, Morokoff, Rossi, \& Meier, 2009), cancer screening behaviors (Spencer, Pagell, \& Adams, 2005; Trauth, Ling, Weissfield, Schoen, \& Hayran, 2003), exercise behaviors (Gorely \& Gordon, 1995; Huang, Hung, Chang \& Chang, 2009; Marcus \& Simkin, 1994; Spencer, Adams, Malone, Roy \& Yost, 2006), organ donation (Frates, Bohrer, \& Thomas, 2006; Weber \& Martin, 2012), mental illness (Velasquez et al., 1999), and HIV prevention (Prochaska, Redding, Harlow, Rossi, \& Velicer, 1994).

Although the TTM has been used to understand a wide variety of health behaviors, it is the theory's use in understanding and applying medical directives that is of particular interest to this study. In a study conducted by Johnson and colleagues (2006), individuals exposed to a stage-matched intervention for cholesterol medication were more likely to move from precontemplation to action and maintenance stages than those who had usual care. Further, these individuals scored significantly higher on measures of adherence to the medication posttreatment. Given the negative relationships of low health literacy and ability in demonstrating how to take medicines and interpreting health messages (Berkman et al., 2011; Lanning \& Doyle, 2010), these findings suggest that the TTM might provide a useful framework for further understanding and eventually developing educational materials regarding low health literacy.

The current application of the TTM focuses on individual decision-making more generally, in that it accounts for and describes how individuals stop negative (i.e., unhealthy) behaviors or adopt positive (i.e., healthy) behaviors by 
progressing through a series of stages. Previous to the TTM, behavior change was often conceptualized as a discrete event, occurring at one point in time. The TTM, however, posits that behavior change is a process that unfolds over time, is nonlinear, and generally follows five stages (Prochaska \& DiClemente, 1983; Slater, 1999). The first stage is precontemplation. In this stage, individuals have no intention to change in the near future, typically operationalized as within the next six months. Further, individuals in the precontemplation stage may have no awareness that they need to change their behavior. In the second stage, contemplation, individuals have recognized that a problem exists and are considering taking action in the near future (i.e., within the next six months), but have not made any actual attempts to do so. In the preparation stage, individuals are in a transition in which they have begun to experiment with or attempt to enact the behavior but have not fully modified their behavior accordingly. Temporally, the preparation stage is thought to involve individuals' consideration of modifying their behavior within the next month. In the action stage, individuals have made specific, overt changes to their behavior within the past six months. In the maintenance stage, the individuals attempt to continue the modifications to their behavior and avoid relapse (Prochaska \& DiClemente, 1983; Slater, 1999).

Most attempts to use the TTM in campaign development have used the stage model to develop materials and persuasive arguments applicable to the current stage of the target audience (Slater, 1999). In this dissertation, the stages of change according to the TTM will be used to provide further evidence of the 
predictive validity of the revised POHLS measure. Specifically, it will be assessed if individual's responses to the revised measure can be used to accurately predict their current "stage" with regard to behaviors known to reduce obesity and diabetes risk, physical activity and healthy eating:

H 7: An individuals' health literacy level can effectively predict his or her placement in the stages of readiness for change regarding physical activity and healthy eating.

\section{Increasing Health Literacy}

A second goal of this manuscript is to assess if health literacy is a construct that can be effectively increased. As discussed by Berkman and colleagues (2010), it is important to distinguish between static and dynamic conceptualizations or treatments of health literacy. Recall that a static or fixed conceptualization of health literacy would imply that individuals' ability to process health information remains relatively stable across adulthood, whereas a dynamic conceptualization of the construct provides the potential for individuals' levels of health literacy to be increased through educational interventions and other forms of skills training (Berkman et al., 2010). The definition of health literacy employed in this study presents the construct as dynamic and able to be influenced. As such, the view that this manuscript takes is that one's ability to process and use health information is in fact a function of cognitive and social skills that can be improved upon via educational efforts and communication.

Treating health literacy as a dynamic construct has important implications for both theory and practical application. If health literacy levels are not stagnant 
and are able to be influenced, then healthcare practitioners, researchers, and patients alike should switch their focus from accommodating low levels of health literacy to instead working to improve these cognitive and social skill sets. To date, interventions on health literacy have primarily focused on simplifying the language and improving the readability of medical documents (e.g., pamphlets on polio, Davis et al., 1998; prescription drug labels, Davis et al., 2006; computermediated messages on diabetes management, Gerber et al., 2005). Yet, as argued by Cortés, Drainoni, Henault, and Paasche-Orlow (2010), even when researchers follow guidelines designed to improve readability (e.g., large font, wide margins, shorter sentences and paragraphs, and plain language) participants’ comprehension of those materials is not guaranteed.

In order to increase comprehension of two sets of medical messages (i.e., patient discharge instructions and promotion of walking among older adults), a clinical trial by Bickmore and colleagues (2010) investigated the utility of a computer interface, the Embodied Conversational Agent, which conveys health information to individuals using an animated character that talks to patients using synthetic speech and synchronized nonverbal behaviors. Patients can "talk back" to the interface using a touch screen input. While the interface received a fairly positive reception from patients in the trials, this interface clearly only applies to the processing of information in the hospital setting (and further, in hospitals which can afford to implement the ECA system).

As Nutbeam (2000) would argue, these interventions have largely taken a functional approach to improving health literacy and have not focused on the 
communicative or critical skills needed to succeed in the healthcare environment. These interventions have been highly content-specific and have not attempted to improve an individual's health literacy in such a way that it would help him or her across a variety of health-related situations. Further, very few interventions have focused on improving cognitive and social skills, with those that have focused on cognitive abilities more generally such as processing speed and working memory (Wilson et al., 2010) rather than improving the cognitive skills related to health literacy more specifically. Importantly, the field of Communication Studies offers a number of practical implications for effectively improving communication skills and subsequent behavior change (Booth-Butterfield, 2003; Cegala, 2006; Cegala \& Broz, 2003; Evans, Stanley, \& Burrows, 1992; Harrington, Norling, Witte, Taylor, \& Andrews, 2007; Hopf \& Ayres, 1992; Kelly, 1989; Rancer, Whitecap, Kosberg, \& Avtgis, 1997; Weber, Martin, \& Patterson, 2001); if it is shown that health literacy can be increased, then researchers and practitioners can apply this knowledge to influence individuals' low healthy literacy and achieve more positive health outcomes. As the interventions that have been conducted to increase health literacy scores have received mixed support (Bickmore et al., 2010; Davis et al., 1998; Gerber et al., 2005; Pignone, DeWalt, Sheridan, Berkman, \& Lohr, 2005; Wilson et al., 2010), the following hypothesis forwards that an intervention aimed to increase individual's scores on the revised POHLS will be successful:

H 8: Individuals exposed to a brief educational intervention on health literacy will have higher change scores in their 
health literacy levels than individuals not exposed to a brief educational intervention on health literacy.

\section{Summary of Chapter I}

This dissertation serves two purposes. First, it seeks to develop a valid and reliable measurement of health literacy based on the World Health Organization (2014) definition of the construct. This new measure will be not only practical for use in the social sciences, but will address both the cognitive and social skills that impact an individual's motivation and ability to gain access to, understand, and use health information to maintain good health (WHO, 2014). The validity of the construct will be argued by its relationship to several theoretically related variables. Second, this dissertation sought to provide evidence that health literacy is a skill set that can be increased through the intervention efforts of communication studies scholars.

This chapter provided an overview of the appropriate literature. Specifically, conceptual definitions of health literacy, the outcomes and prevalence of low health literacy, and the current dilemma facing social scientists regarding the measurement of health literacy were reviewed. A clear statement of the problem was presented. Finally, a rationale for the research question and eight hypotheses of this dissertation was provided. 


\section{CHAPTER II}

\section{Overview}

\section{Methods}

The research question and eight hypotheses of this dissertation were addressed in four studies. Study One addressed the research question. Study Two addressed hypotheses one, two, three, four, and eight. Study Three addressed hypothesis five. Study Four addresses hypotheses six and seven.

\section{Study One}

Study One looked to further develop the Perceived Oral Health Literacy Scale (LaBelle \& Weber, 2013) by examining how the scale could be refined to more accurately represent an individual's motivation and ability to gain access to, understand, and use health information (WHO, 2014). Due to the concern that the three factors of the existing scale (i.e., Question Asking, Adequacy of Information, and Ability to Apply Medical Directives) may reflect a participants’ satisfaction with a healthcare provider, Study One sought to develop items which would reflect a wider spectrum of health literacy as provided in the WHO (2014) definition.

The decision to add items to the POHLS is based upon sound practices and knowledge from the field of psychometrics regarding the internal consistency and reliability of scales (see DeVellis, 2012). The internal consistency reliability of a scale is based on how strongly the items correlate with one another as well as how many items are in the scale; that is, more items that are similar in nature allow measures to be more stable and consistent. Although the role that participant fatigue plays in responding to survey items should not be understated, the importance of scale reliability should (within 
reason) outweigh the desire for brevity (DeVellis, 2012). Further, it is a generally accepted rule in developing measure for social science that a factor should ideally be represented by three or more items (Hatcher, 1994; Weber \& Patterson, 1996). Given that the third factor, Ability to Apply Medical Directives, was only represented by two items in LaBelle and Weber's (2013) study, it would benefit the measure to add more items per factor in its revision.

\section{Participants and Procedures}

In accordance with the guidelines for item development forwarded by Cohen, Swerdlik, and Sturman (2010), 51 additional items were developed to represent individuals’ motivation and ability to gain access to, understand, and use health information. Items were written which also reflected specific behaviors that represent an individuals' health literacy (e.g., "I use the health information I see and hear in reference to my own life”). In order to make sure that items were developed which met the conceptualization of health literacy being used in the study, a 3 x 3 matrix was constructed in which items were written to reflect individuals’ (1a) motivation, (2a) ability, and (3a) behaviors to (1b) gain access to, (2b) understand, and (2c) use health information.

These items, along with the original item pool of the POHLS $(n=20)$, were pilot tested for their readability and clarity (see Appendix F for the pilot test questionnaire). The pilot test was administered to three volunteer samples in order to achieve a wide range of feedback on the scale. The first sample $(n=17)$ consisted of individuals at a local community center, the second sample $(n=56)$ of undergraduate students at a large Mid-Atlantic university, and a third network-based sample $(n=3)$ for a total of 76 
participants in the pilot test. Participants for this study ranged in age from 19 to $65(M=$ 25.26, $S D=10.22)$. There were $29(39.7 \%)$ males and 44(60.3\%) females and 3 nonreports. The majority $(n=68)$ of participants were Caucasian $(89.5 \%)$, with 3 Hispanic (3.9\%), 2 Asian (2.6\%), and 2 Black/African-American (2.6\%) participants. One participant (1.3\%) described his/her ethnicity as "Other.” The participants provided feedback on the instructions and the design of the questionnaire, as well as the item construction and readability. This feedback was incorporated into the measure that was administered for Study One, which included adding an additional three items to the questionnaire. Additionally, these items were assessed for their readability using the Flesch-Kincaid Grade Level and Reading Ease indices ${ }^{2}$. The Flesch-Kincaid Grade Level for the items was 5.7 and the Flesch Reading Ease Test was 68. A Flesch-Kincaid Grade Level of 5.7 means that a fifth-sixth grade student can understand the items and the Reading Ease index falls within an acceptable range of average sentence length and number of syllables per word. All 74 items used in Study One can be found in Appendix G.

Following the pilot test and approval by the university’s institutional review board, undergraduate Communication Studies classrooms were entered to distribute a written questionnaire. Participation was anonymous and voluntary. Participants for this study consisted of a convenience sample of 444 undergraduate students from Communication Studies courses at a large mid-Atlantic university. Participants ranged in age from 18 to $45(M=19.72, S D=2.20)$. There were $243(56.1 \%)$ males and 190 (43.9\%) females and 11 non-reports. The majority $(n=345)$ of participants were Caucasian (78.6\%), with 45 African-American (10.3\%), 17 Hispanic (3.9\%), 10 Asian 
(2.3\%), 8 Middle Eastern (2.3\%), and 4 Native American (.9\%) participants. Eight participants (1.8\%) described their ethnicity as “Other.” Participants included 158 firstyear (35.9\%), 112 sophomore (25.5\%), 99 junior (22.5\%), 66 senior (15.0\%) students, and 5 (1.1\%) graduate students. Four participants indicated their academic status as "Other” (.9\%). The recruitment script, cover letter, and questionnaire used in this study can be found in Appendices H-J.

\section{Measurement}

Health literacy. Perceived health literacy was assessed using the 74 items created to measure individuals' motivation and ability to gain access to, understand, and use health information. Items were measured on a 5 point Likert-type scale ranging from 1 (Strongly Disagree) to 5 (Strongly Agree). Higher scores indicate higher perceptions of health literacy.

Demographic information. Participants provided information regarding their age, sex, ethnicity, and current year in college (i.e., freshman, sophomore, junior, senior, graduate, or other).

\section{Data Analysis}

An exploratory factor analysis was conducted in a statistical software program (i.e., SPSS 19) in order to determine the underlying dimensions and latent factors (Tabachnick \& Fidell, 2013) across the initial item pool. Preliminary criteria for retention included examination of Scree plots, minimum eigenvalues greater than 1.0, a variance accounted for of over $5 \%$, and primary factor loadings at, or greater than .50 and less than .20 between the primary and secondary loading (Field, 2009; Hatcher, 1994; Weber \& Patterson, 1996; Tabachnick \& Fidell, 2013). 


\section{Study Two}

Study Two tested hypotheses one, two, three, four, and eight. Specifically, Study Two aimed to establish the content and construct validity of the Perceived Oral Health Literacy Scale (POHLS), and to assess if individuals' levels of health literacy according to the POHLS could be increased following an educational training session. To this end, Study Two has two parts: Phase One was designed to assess the content and construct validity of the POHLS, while Phase Two was designed to assess whether health literacy is a construct that can be effectively increased.

\section{Participants and Procedures}

Participants for this study consisted of a convenience sample of 208 undergraduate students from Interpersonal Communication Studies courses at a large mid-Atlantic university. There were 141 (67.8\%) participants in the experimental group and 67 (32.2\%) students in the control group. Participants ranged in age from 18 to 33 ( $M$ $=20.12, S D=1.86)$. There were $107(51.4 \%)$ male and $101(48.6 \%)$ female participants. One participant did not report his or her sex. The majority $(n=174)$ of participants were Caucasian (84.1\%), with 17 African-American (8.2\%), 7 Middle Eastern (3.4\%), 6 Asian (2.9\%), and 2 Hispanic (1.0\%) participants. One participant (.5\%) described his/her ethnicity as "Other" and there was one non-report. Participants included 46 first-year (22.1\%), 76 sophomore (36.5\%), 49 junior (23.6\%), 35 senior (16.8\%) students, and one (.5\%) graduate student. One participant indicated his/her academic status as "Other" (.5\%). A report of these demographics broken down by experimental condition is provided in Table 3.

Following approval by the university institutional review board, a pretest-posttest 
Table 3

Study Two Demographic Data Categorized by Experimental Condition

\begin{tabular}{|c|c|c|}
\hline & Experimental Group $^{\mathrm{a}}$ & Control Group ${ }^{b}$ \\
\hline Age* & $M=20.23, S D=2.02$ & $M=19.86, S D=1.41$ \\
\hline Sex & 70 Male, 71 Female & 37 Male, 30 Female \\
\hline \multirow[t]{6}{*}{ Ethnicity* } & 118 White/Caucasian & 56 White/Caucasian \\
\hline & 10 Black/African-American & 7 Black/African-American \\
\hline & 6 Middle Eastern & 1 Middle Eastern \\
\hline & 5 Asian & 1 Asian \\
\hline & 1 Hispanic & 1 Hispanic \\
\hline & 1 “Other” & 0 “Other” \\
\hline \multirow[t]{6}{*}{ Class Rank } & 36 First Year & 10 First Year \\
\hline & 45 Sophomore & 31 Sophomore \\
\hline & 34 Junior & 15 Junior \\
\hline & 24 Senior & 11 Senior \\
\hline & 1 Graduate & 0 Graduate \\
\hline & 1 “Other” & 0 “Other” \\
\hline
\end{tabular}

Note. ${ }^{\mathrm{a}} \mathrm{N}=141,{ }^{\mathrm{b}} \mathrm{N}=67 .{ }^{*}$ Missing case in data. 
nonequivalent groups design was employed in which two sections of an introductory Interpersonal Communication Studies course were designated to be either an experimental or a control group. In Phase One of this study undergraduate Communication Studies courses were entered to distribute written questionnaires. Participation was voluntary and confidential. One week later, Phase Two of the study occurred in which a brief educational presentation occurred and a second written questionnaire was administered. The experimental group was exposed to a 35 minute presentation on the dimensions of health literacy and the importance of being motivated and able to gain access to, understand, and use information in order to promote and maintain good health whereas the control group was given an equal length presentation on an unrelated topic (see Appendix $\mathrm{N}$ for the notes that were used in these educational presentations). These educational presentations were delivered by the same member of the research team and on the same day in order to control for extraneous variables such as teaching style and time of the semester. Following the presentation, each group was given the second written questionnaire. The items on both the first and second questionnaires were identical; individual participants’ responses were linked by a unique code number. The Phase One questionnaires constituted the pretest data for the relevant analyses below; the Phase Two questionnaires constituted the posttest data. The recruitment scripts, cover letters, measures, questionnaire, and educational intervention notes used in Phase One and Phase Two of this study can be found in Appendices L-T.

\section{Measurement}

Health literacy. Health literacy was operationalized using a revised 26-item Perceived Oral Health Literacy Scale (POHLS) developed in Study One ${ }^{1}$. The scale 
consists of two factors which assess individual's motivation and ability to gain access to, understand, and use health information. Items were measured on a 5 point Likert-type scale ranging from 1 (Strongly Disagree) to 5(Strongly Agree). Higher scores indicate higher perceptions of health literacy. Sample items include "I am motivated to seek information which will improve my health," (motivation) and "I understand the terms a healthcare provider uses in interactions with me” (ability). For Phase One of this study, Cronbach's alpha reliability coefficients of $.89(M=50.01, S D=7.70)$ and $.85(M=$ $51.58, S D=6.39)$ were obtained for the motivation and ability subscales, respectively. In Phase Two, Cronbach's alpha reliability coefficients of $.89(M=50.12, S D=8.16)$ and $.88(M=50.21, S D=7.63)$ were obtained for the motivation and ability subscales.

Perceived health competence. Perceived health competence was operationalized using the 8-item Perceived Health Competence Scale (Smith et al., 1995). Items were measured on a 5 point Likert-type scale ranging from 1 (Strongly Disagree) to 5 (Strongly Agree). Higher scores on the scale indicate higher perceptions of perceived health competence. Items on the measure include "I handle myself well with respect to my health” and "I succeed in the projects I undertake to improve my health.” This scale has been administered previously with an adequate reliability coefficient ( $\alpha=.88$; Waldrop et al., 2001). For Phase One of this study, a Cronbach’s alpha reliability coefficient of .83 was obtained for the measure $(M=29.08, S D=4.75)$. In Phase Two, a Cronbach's alpha reliability coefficient of .83 was obtained $(M=28.94, S D=5.18)$.

Patient efficacy. Health efficacy in interactions with a healthcare provider was operationalized using a 6-item measure of patient efficacy (Young \& Klingle, 1996). This measure consists of two dimensions assessing both self and response efficacy in 
interacting with a physician during a medical interview (Young \& Klingle, 1996). Items were measured on a 5 point Likert-type scale ranging from 1 (Strongly Disagree) to 5 (Strongly Agree). Higher scores on the scale indicate higher levels of self and response efficacy. Sample items include "I think it would be easy to participate in medical decision-making with my physician,” (self efficacy) and “Open communication with my physician will improve the quality of my medical care” (response efficacy). This measure has been administered previously with reliability coefficients approaching acceptability for both the self $(\alpha=.62)$ and response $(\alpha=.63)$ components (Young \& Klingle, 1996). For Phase One of this study, Cronbach’s alpha reliability coefficients of $62(M=11.83, S D=2.12)$ and $.72(M=12.53, S D=1.99)$ were obtained for the self and response efficacy subscales, respectively. In Phase Two, Cronbach’s alpha reliability coefficients of $.64(M=11.66, S D=2.25)$ and $.85(M=12.70, S D=2.21)$ were obtained for the self and response efficacy subscales.

Communication anxiety. Communication Anxiety was operationalized using the Form Trait subscale of the Communication Anxiety Inventory (Booth-Butterfield \& Gould, 1986). This measure assesses individuals’ trait communication anxiety as well as their anxiety in three contexts: dyadic, small group, and public speaking (BoothButterfield \& Gould, 1986). Items were measured on a 5 point Likert-type scale ranging from 1 (Strongly Disagree) to 5 (Strongly Agree). Higher scores on the summed measure indicate higher trait communication anxiety; higher scores on each of the three subscales indicate higher communication anxiety in that context. Sample items include "I think I communicate effectively in one-to-one situations," (dyadic), "My heart beats faster than usual when I speak out in a small group meeting," (small group), and “I am terrified at 
the thought of speaking in public" (public speaking). This measure has been administered previously with adequate reliability coefficients for the summed measure ( $\alpha$ $=.90)$, as well as the dyadic $(\alpha=.65)$, small group $(\alpha=.85)$, and public speaking $(\alpha=$ .89) subscales (Booth-Butterfield \& Gould, 1986). For Phase One of this study, Cronbach's alpha reliability coefficients of $.90(M=61.10, S D=12.59), .75(M=18.37$, $S D=4.24), .84(M=20.60, S D=5.12)$, and $.85(M=22.17, S D=5.62)$ were obtained for the summed measure and the dyadic, small group, and public speaking subscales, respectively. In Phase Two, Cronbach’s alpha reliability coefficients of .91 ( $M=59.98$, $S D=13.63), .71(M=18.66, S D=4.29), .81(M=19.54, S D=5.34)$, and $.86(M=21.79$, $S D=5.93$ ) were obtained for the summed measure and the dyadic, small group, and public speaking subscales.

Demographic information. Participants provided information regarding their age, sex, ethnicity, and current year in college (i.e., freshman, sophomore, junior, senior, graduate, or other).

\section{Data Analysis}

Kerlinger (1986) argued that determining content validity is largely a test of judgment, and confirmatory factor analysis provides an empirical judgment to validate the number of factors that exist but also their goodness of fit (Hatcher, 1994; Tabachnick \& Fidell, 2013). Therefore, in order to provide an argument for the content validity of the measure, and therefore test hypothesis one, a confirmatory factor analysis was performed on using a statistical software program (i.e., AMOS 19). Notably, six participants were eliminated from the data set used for running the confirmatory factor analysis due to missing one or more cases. The adequacy of model fit was determined by 
examining the model Chi Square to degree of freedom ratio, the Standardized Root Mean Squared Residual (SRMR; Hu \& Bentler, 1999), and Root Mean Squared Error of Approximation (RMSEA; Stieger \& Lind, 1980). For the ratio of the model chi square to its degrees of freedom, a value of below 3 was considered to be indicative of good fit (Byrne, 2001; Kline, 2011). A SRMR value close to .08 was considered indicative of good fit (Hu \& Bentler, 1999), as was an RMSEA value no greater than .08 (Browne \& Cudeck, 1993; MacCallum, Browne, \& Sugawara, 1996). Hypotheses two, three, and four were assessed using Pearson Product-Moment Correlations in SPSS 19. Hypothesis eight was assessed by first calculating change scores on participants' health literacy levels from the pretest to the posttest and then conducting an Independent Samples T-test to determine if the change scores of the experimental and control groups were significantly different.

\section{Study Three}

Study Three sought to test hypothesis five. Specifically, Study Three aimed to further establish the construct validity by providing a test of the known groups validity of the revised Perceived Oral Health Literacy Scale (POHLS). Although seemingly simple in its theoretical basis and practical application, known groups validity is crucially important to establishing the construct validity of a scale (Eagly \& Chaiken, 1993). For instance, if one were to develop a measure of attitudes toward abortion that could not differentiate between individuals in pro-life and pro-choice groups, Eagly and Chaiken (1993) argued that the evidence for the validity of the scale would be severely compromised. Given the importance of establishing the known-groups validity of a measure, the 
third study investigates whether the revised POHLS can distinguish between two groups of individuals who should, theoretically, score differently on the scale. To do so, the health literacy scores of individuals who have took part in a semester long course in Health Communication were compared to the scores of individuals who did not take part in a health-related course. This provided evidence of a third type of construct validity for the measure.

\section{Participants and Procedures}

Following approval by the university institutional review board, participants were recruited from two undergraduate courses in Communication Studies. One group of students was enrolled in a Health Communication course; the other group consisted of participants enrolled in either an Advanced Interpersonal course or a Business Communication course. In the last week of the semester, classrooms were entered to distribute written questionnaires. There were no participants who indicated an overlap in taking the courses, and participants in the Advanced Interpersonal and Business Communication courses had not previously taken a Health Communication course. Participation was confidential and voluntary. The recruitment script, cover letter, and questionnaire used in this study can be found in Appendices U-W.

Participants for this study consisted of a convenience sample of 59 undergraduate students from Communication Studies courses at a large mid-Atlantic university. There were 25 (42.4\%) students in the Health Communication course and 34 (57.6\%) students in the Advanced Interpersonal Communication and Business Communication courses. Participants ranged in age from 20 to $34(M=21.76, S D=2.28)$. There were $25(42.4 \%)$ male and $34(57.6 \%)$ female participants. The majority $(n=50)$ of participants were 
Caucasian (84.7\%), with 5 African-American (8.5\%), and 2 Hispanic (3.4\%) participants. Two participants (3.4\%) described their ethnicity as “Other.” Participants included one sophomore (1.7\%), 17 junior (28.8\%), and 41 senior (69.5\%) students. A report of these demographics broken down by group (i.e., Health Communication or non-Health Communication) is provided in Table 4.

\section{Measurement}

Health literacy. Health literacy was operationalized using a revised 26-item Perceived Oral Health Literacy Scale developed in Study One. The scale consists of two factors which assess individual's motivation and ability to gain access to, understand, and use health information. Items were measured on a 5 point Likert-type scale ranging from

1 (Strongly Disagree) to 5 (Strongly Agree). Higher scores indicate higher perceptions of health literacy. Sample items include "I am motivated to seek information which will improve my health" (motivation) and "I understand the terms a healthcare provider uses in interactions with me” (ability). In this study, Cronbach alpha reliability coefficients of $.91(M=52.08, S D=7.38)$ and $.85(M=52.14, S D=5.82)$ were obtained for the motivation and ability subscales, respectively.

Demographic information. Participants provided information regarding their age, sex, ethnicity, and current year in college (i.e., freshman, sophomore, junior, senior, graduate, or other).

\section{Data Analysis}

Hypothesis five was assessed using an Independent Samples t -test conducted in a statistical software program (i.e., SPSS 19). 
Table 4

Study Three Demographic Data Categorized by Group

\begin{tabular}{|c|c|c|}
\hline & $\begin{array}{l}\text { Health Communication } \\
\text { Course }^{\text {a }}\end{array}$ & $\begin{array}{l}\text { Non-Health Communication } \\
\text { Course }^{\text {b }}\end{array}$ \\
\hline \multicolumn{3}{|c|}{ 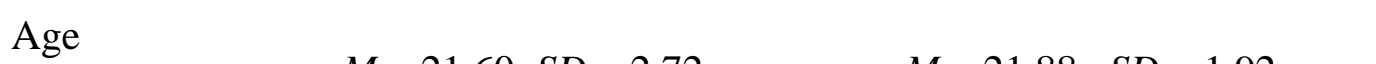 } \\
\hline Sex & 6 Male, 19 Female & 19 Male, 15 Female \\
\hline \multirow[t]{4}{*}{ Ethnicity } & 22 White/Caucasian & 28 White/Caucasian \\
\hline & 2 Black/African-American & 3 Black/African-American \\
\hline & 0 Hispanic & 2 Hispanic \\
\hline & 1 “Other” & 1 “Other” \\
\hline \multirow[t]{3}{*}{ Class Rank } & 1 Sophomore & 0 Sophomore \\
\hline & 7 Junior & 10 Junior \\
\hline & 17 Senior & 24 Senior \\
\hline
\end{tabular}

Note. ${ }^{\mathrm{a}} \mathrm{N}=25,{ }^{\mathrm{b}} \mathrm{N}=34$. 


\section{Study Four}

Study Four aimed to test hypotheses six and seven. Specifically, this study sought to provide evidence of the criterion-related validity of the revised Perceived Oral Health Literacy Scale (POHLS) in two ways. First, this study determined if an individual's score on the measure could predict physiological health indicators of risk for obesity and diabetes. Second, the Transtheoretical Model (Prochaska \& DiClemente, 1983) was employed to determine if the revised POHLS could successfully predict an individual's stage of change regarding behaviors related to physical activity and healthy eating.

Notably, Study Four deviates from the previous three studies in examining the role of health literacy in a specific context: obesity and diabetes prevention. This study also uses a purposive sample; adult residents of West Virginia constituted the participants for Study Four, as this population is highly at risk for these health issues. As reported by the CDC (2013a), the prevalence of obesity in the United States varies by region, with thirteen states having a prevalence of obesity equal to or above 30 percent (i.e., Alabama, Arkansas, Indiana, Iowa, Kentucky, Louisiana, Michigan, Mississippi, Ohio, Oklahoma, South Carolina, Tennessee, and West Virginia). To focus on a group of people that is particularly at risk for issues related to obesity and diabetes, the sample for Study Four consisted exclusively of West Virginia residents. Approximately $12 \%$ of adults in West Virginia have been diagnosed with diabetes by a healthcare professional, and with an additional 85,500 individuals remaining undiagnosed, this disease continues to be a leading cause of death in the state (Health Statistics Center, HSC, 2012). West Virginians also struggle with a high prevalence of obesity, physical inactivity, hypertension, and hyperlipidemia, all of which increase risk for developing Type II 
diabetes (HSC, 2012). According to a 2010 survey on the health risks of West Virginians, nearly 33\% of the state's residents do not participate in any form of physical activity outside of what is required by their regular occupation, with $35 \%$ of these individuals reporting a BMI of 30.0 or more (i.e., obese) and the largest percentage of tobacco use in the country (HSC, 2012). Overall, West Virginians have the fourth highest prevalence of diabetes among the 50 states, and self-report fair or poor health status (HSC, 2012). Diabetes is also related to social risks, in that diabetes is highest among those with an annual household income of less than \$15,000 (Manchin, Hardy, Curtis, Bazzle, \& Slump, 2009); this is critical when considering that 17.6 percent of West Virginians live below the poverty level (U.S. Census Bureau, 2012). It is highly likely, then, that understanding the role of health literacy in affecting individuals’ cognitions, attitudes, and physiological health indicators related to obesity and diabetes will offer a more comprehensive understanding of the epidemic of poor health affecting this region.

\section{Participants and Procedures}

Participants for this study consisted of a purposive sample of 40 adult visitors of a community center in North Central West Virginia. Participants ranged in age from 18 to $63(M=31.36, S D=10.31)$. All 40 participants were female. The majority $(n=30)$ of participants were Caucasian (75.0\%), with 4 Asian (10.0\%), 3 African-American (7.5\%), and 3 Hispanic $(7.5 \%)$ participants. The majority $(n=23)$ of participants were married (57.5\%), and $12(30.0 \%)$ were single, 4 were engaged or in a committed relationship (10.0\%), and one participant was divorced (2.5\%). The majority $(n=12)$ of participants had a yearly household income of $\$ 0-\$ 10,000$ (30.0\%), two participants (7.5\%) reported 
an income of $\$ 10,001-\$ 20,000$, six participants (15.0\%) reported a yearly household income of $\$ 40,001-\$ 60,000$, two participants (7.5\%) reported an income of $\$ 60,001$ $\$ 80,000$, six reported (15.0\%) an income of $\$ 80,001-\$ 100,000$, four participants (10.0\%) reported an income of $\$ 100,001-\$ 120,000$, and one participant reported a yearly household income of $\$ 120,001-\$ 140,000$. There were no participants that reported a yearly household income in the $\$ 20,001-\$ 40,000$ range. Eighteen participants earned a college degree (48.6\%), with 10 participants reporting their highest degree was a high school diploma (27.0\%), four held a Master’s degree (10.8\%), three had a professional or trade certificate (8.1\%), one participant held a doctorate (2.7\%), one participant held a general education diploma (2.7\%), and three participants (8.1\%) did not report their highest degree earned.

Following approval by the university institutional review board, participants were recruited from a community center with the help of its volunteer coordinators and staff. The community center used for recruitment was selected due to its active role in serving families in North Central West Virginia. Recruitment materials (i.e., flyers and copies of the recruitment script) were distributed at the community center to promote a free health screening. Two 3 hour screenings were conducted in which participants completed written questionnaires. A registered LPN on staff at the community center administered a blood glucose level measurement and calculated participants’ Body Mass Index. Participants were provided a monetary incentive (i.e., a gift certificate) to participate. Participation was confidential and voluntary. Participants underwent a consent procedure in accordance with university requirements, which required the signature of both the 
participant and researcher. The recruitment script, recruitment flyer, consent form, cover letter, measures, and questionnaire used in this study can be found in Appendices X-DD.

\section{Measurement}

Health literacy. Health literacy was operationalized using a revised 26-item Perceived Oral Health Literacy Scale developed in Study One. The scale consists of two factors which assess individual's motivation and ability to gain access to, understand, and use health information. Items were measured on a 5 point Likert-type scale ranging from 1 (Strongly Disagree) to 5 (Strongly Agree). Higher scores indicate higher perceptions of health literacy. Sample items include "I am motivated to seek information which will improve my health" (motivation) and “I understand the terms a healthcare provider uses in interactions with me” (ability). In this study, Cronbach alpha reliability coefficients of $.89(M=51.80, S D=7.35)$ and $.84(M=50.95, S D=6.54)$ were obtained for the motivation and ability subscales, respectively.

Physiological health indicators. Physiological indicators of health were operationalized using individual's blood glucose levels as well as his or her Body Mass Index (BMI), as these are known to be indicators of risk for diabetes and obesity (National Diabetes Education Program, 2014). The FreeStyle Freedom Lite Blood Glucose Monitoring System was used by a registered LPN to obtain a blood glucose level for each participant. This meter displays results from $20-500 \mathrm{mg} / \mathrm{dL}$, with a glucose level lower than $60 \mathrm{mg} / \mathrm{dL}$ indicating hypoglycemia (i.e., low blood sugar levels) and higher than $240 \mathrm{mg} / \mathrm{dL}$ indicating hyperglycemia (i.e., high blood sugar). The average glucose level obtained in this study was $90.73 \mathrm{mg} / \mathrm{dL}(S D=20.79$, Range $=74-128$ 
mg/dL). Therefore, no participants in the study were either hypoglycemic or hyperglycemic.

An individual's BMI is a number calculated from his or her weight and height. BMI provides a reliable indicator of body fat percentage for most people, and is often used to screen for weight categories associated with health problems (Center for Disease Control, CDC, 2013b). The registered LPN on staff at the community center asked for participants' height and weight. Each participant's BMI was then calculated and recorded on the written questionnaire. Specifically, a BMI is calculated by dividing weight in pounds by height in inches squared and multiplying by a conversion factor of 703 (i.e., weight (lb) / [height (in)] ${ }^{2}$ x 703; CDC, 2011b). An adult BMI of below 18.5 indicates that one is underweight; a BMI of 18.5-24.9 indicates a normal weight status; a BMI of 25.0-29.9 indicates that one is overweight, and a BMI of 30.0 or above indicates that one is obese. In this study, 14 (38.9\%) participants had a normal weight status, 13 (36.1\%) were overweight, and 9 (25.0\%) were obese. The average BMI obtained in this study was $27.95(S D=6.97$, Range $=19.96-49)$.

Stages of Change. Stages of change regarding behaviors related to physical activity and healthy eating was assessed with two items developed based on previous research on the Transtheoretical Model (e.g., Sarkin, Johnson, Prochaska, \& Prochaska, 2001; Vallis et al., 2003). Following the methodology of Sarkin and colleagues (2001) and Vallis et al. (2003), a staging algorithm was used which focused on individuals' behaviors related to physical activity and healthy eating, behaviors related to risk for diabetes (Manchin et al. 2009). Participants were provided with a description of the behaviors under study, which was based on the World Health Organization's (2010) 
definition of physical activity as well as previous research using the TTM in the context of healthy eating (Sarkin et al., 2001; Vallis et al., 2003). The description provided to participants read:

The following questions concern your current behaviors related to physical activity and healthy eating. For this study, physical activity includes at least 150 minutes of moderate-intensity aerobic physical activity throughout the week or at least 75 minutes of vigorous-intensity aerobic physical activity throughout the week or an equivalent combination of moderate- and vigorous-intensity activity. Healthy eating refers to a diet consisting of low fat and low calorie foods. Following this description, participants were asked to select one of five options regarding their intentions to engage in physical activity (Item 1) and healthy eating (Item 2). Depending on an individual's response, he or she was classified into one of the five stages of change: (1) Precontemplation (e.g., "No, I do not do the amount of physical activity indicated above, and I do not intend to in the next six months”), (2) Contemplation (e.g., "No, I do not do the amount of physical activity indicated above, but I intend to in the next six months”), (3) Preparation (e.g., “No, I do not do the amount of physical activity indicated above, but I intend to in the next 30 days”), (4) Action (e.g., "Yes, I regularly do the amount of physical activity indicated above, but I have been doing so for less than six months”), or (5) Maintenance (e.g., "Yes, I do the amount of physical activity indicated above, and I have been doing so for more than six months”). The frequency of participants in each stages of change category for both physical activity and healthy eating are provided in Table 5. 
Table 5

Frequency of Stages of Change for Physical Activity and Healthy Eating

Physical Activity $^{\mathrm{a}} \quad$ Healthy Eating $^{\mathrm{b}}$

$\begin{array}{lll}\text { Precontemplation } & n=2 & n=6 \\ \text { Contemplation } & n=11 & n=6 \\ \text { Preparation } & n=11 & n=4 \\ \text { Action } & n=9 & n=10 \\ \text { Maintenance } & n=7 & n=14\end{array}$

Note. ${ }^{\mathrm{a}} \mathrm{N}=40,{ }^{\mathrm{b}} \mathrm{N}=40$. 
Demographic information. Participants provided information regarding their age, sex, ethnicity, marital status, income, and education.

\section{Data Analysis}

The sixth hypothesis was analyzed using a Pearson Product-Moment Correlation. The seventh hypothesis was analyzed using a discriminant function analysis, which is able to predict group membership based upon a continuous variable (Tabachnick \& Fidell, 2013). These analyses will be conducted in a statistical software program (i.e., SPSS).

\section{Summary of Chapter II}

This chapter provided an overview of the methodologies used for this dissertation. The various measures employed in each of the four studies were described in detail in term of items, response categories, and reliability coefficients. This chapter also provided demographic information regarding the participants of these four studies and the recruitment procedures that were used to obtain these participants. Finally, this chapter included a description of the data analysis procedures used to answer the research question and eight hypotheses of this dissertation. 


\section{CHAPTER III}

\section{Results}

The first research question inquired if the Perceived Oral Health Literacy Scale (POHLS) developed by LaBelle and Weber (2013) could be further refined to more accurately represent the World Health Organization's (2014) definition of health literacy as an individual's motivation and ability to gain access to, understand, and use health information in order to promote good health. As outlined in Chapter II, answering this research question involved developing an additional 54 items using a 3 x 3 matrix designed to reflect individuals' (1a) motivation, (2a) ability, and (3a) behaviors to (1b) gain access to, (2b) understand, and (2c) use health information. These 54 items, in addition to the 20 items that were a part of the original POHLS item pool, were subjected to an exploratory factor analysis using a principal axis factoring extraction and an oblique (i.e., promax) rotation.

The Kaiser-Meyer-Olkin test of sampling adequacy (.95) and Bartlett’s test of sphericity $\left(\chi^{2}(2701)=16518.06, p<.001\right)$ indicated that factor analysis was appropriate for the data in the preliminary item pool. In the initial factor analysis, 32 items were removed due to poor primary factor loadings (i.e., below .5; Field, 2009; Hatcher, 1994). The remaining items were again subjected to factor analysis, and 11 items were deleted due to poor secondary loadings (i.e., less than .20 between primary and secondary loading; Field, 2009; Hatcher, 1994; Weber \& Patterson, 1996). The final iteration consisted of 32 of the original 74 items, and suggested the presence of a two, four, or six factor solution. Specifically, an examination of the Scree Plot shown in Figure 2 provides evidence of a two, four, or six factor solution depending on the relatively 
Figure 2

Scree Plot for Exploratory Factor Analysis

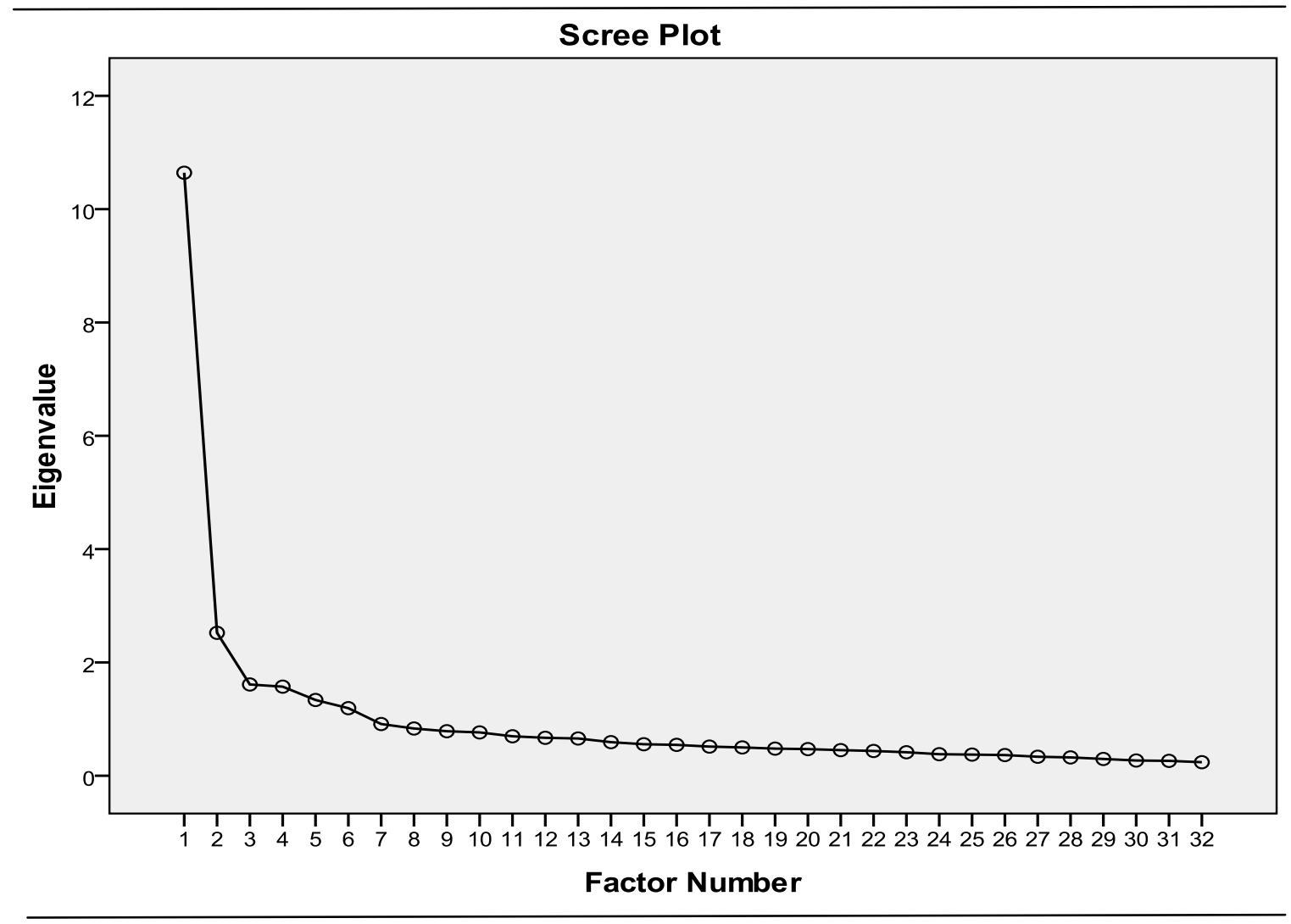


subjective assessment of where the "break" in the graphical eigenvalue illustration can be found (Field, 2009; Tabachnick \& Fidell, 2013).

As there was potential in this Scree Plot for six factors, the remaining criteria for all six factors will be provided followed by an interpretation of what collectively these criteria suggest for the final factor solution. Factor One accounted for $31.72 \%$ of the variance $($ Eigenvalue $=10.15)$ and consists of 10 items that yield a Cronbach's alpha reliability coefficient of $.90(M=37.84, S D=6.48)$. Factor Two accounted for $6.36 \%$ of the variance (Eigenvalue $=2.03$ ) and consists of 9 items that yield a Cronbach's alpha reliability coefficient of $.88(M=35.09, S D=5.33)$. Factor Three accounted for 3.58\% of the variance (Eigenvalue $=1.14)$ and consists of 3 items that yield a Cronbach's alpha reliability coefficient of $.77(M=10.54, S D=2.79)$. Factor Four accounted for $3.30 \%$ of the variance (Eigenvalue $=1.06$ ) and consists of 4 items that yield a Cronbach's alpha reliability coefficient of $.72(M=14.72, S D=2.98)$. Factor Five accounted for $2.58 \%$ of the variance (Eigenvalue $=.82$ ) and consists of 3 items that yield a Cronbach's alpha reliability coefficient of $.74(M=11.78, S D=2.22)$. Factor Six accounted for $2.27 \%$ of the variance (Eigenvalue $=.72$ ) and consists of 3 items that yield a Cronbach's alpha reliability coefficient of $.78(M=12.09, S D=2.31)$.

Per the criteria set forth for determining factors in this study (Field, 2009; Hatcher, 1994), the variance accounted for by each factor therefore suggested a two factor solution, as only Factor One and Factor Two accounted for more than $5 \%$ of the variance. However, four additional factors derived an eigenvalue larger than 1 and had at least three items with their primary loadings, which could suggest the presence of six factors. 
The items loading on each factor were then closely examined to determine commonalities or themes. The items loading on Factor One (i.e., Items 10, 30, 32, 41, $43,49,64,65,71$, and 72 ) concern an individual's motivation to gain access to, understand, and use health information to achieve or maintain good health (e.g., "I am motivated to use information that I receive to improve my health”). This factor was therefore named Motivation. The items loading on Factor Two (i.e., Items 31, 34, 38, 40, 46, 50, 51, 52, and 54) concern an individual's ability to understand and use health information (e.g. "I am able to understand discussions about health and healthy living”). This factor was therefore named Ability. The items loading on Factor Three (i.e., Items 60,66 , and 67) reflected a lack of motivation to understand and use health information (e.g., "I am okay with not understanding everything a healthcare provider is talking about regarding my health”). This factor was therefore named Lack of Motivation. The items loading on Factor Four (i.e., Items 14, 15, 16, and 22) reflect an individual's lack of ability to understand and use health information (e.g., "I have a hard time using suggesting I hear on how to live a healthy lifestyle”). This factor was therefore named Lack of Ability. It needs to be noted that all items on both Factors Three and Four are recoded items that were originally conceptualized to load with the items on Factors One and Two.

The items loading on Factor Five (i.e., 3, 11, and 13) concern an individual's ability to ask a healthcare provider questions (e.g., "I feel confident in my ability to ask a healthcare provider questions”). Therefore this factor was named Competence in Interactions with a Healthcare Provider. The items loading on Factor Six (i.e., Items 59, 63, and 73) concern an individual's ability to gain access to healthcare when needed (e.g., 
"I am able to seek healthcare (e.g., talk to a doctor) when I need it”). This factor was therefore named Competence in Finding a Healthcare Provider.

These six factors were then examined for their relation to and representativeness of the WHO (2014) definition of health literacy: "the cognitive and social skills which determine the motivation and ability of individuals to gain access to, understand, and use information in ways which promote and maintain good health” (“Track 2: Health literacy and health behaviour,” para. 1). In doing so, it is apparent that although the first four factors assess components of an individual's motivation and ability to gain access to, understand, and use health information in order to promote good health, Factors Five and Six (i.e., Competence in Interactions with a Healthcare Provider and Competence in Finding a Healthcare Provider) do not directly represent the construct that this measure aims to operationalize. Rather, the way that these items grouped together in the factor analysis seem to suggest a wording bias: all six items represented by these two factors relate to interactions with a specific "healthcare provider." Therefore, it is possible that the items on these two factors could all potentially be confounded by the quality of one's healthcare provider and not necessarily represent the motivation and ability of the individual completing the scale. For instance, the item "I feel confident in my ability to ask a healthcare provider questions” might elicit a response of "strongly disagree” from an individual who is both highly motivated and able to ask questions, but who does not have quality interactions with his or her provider. This particular item was in fact on the original POHLS measure, and is a representative example of LaBelle and Weber's (2013) concern that the quality of one's healthcare provider, and not his or her own health literacy, could have driven the factors that emerged in the original iteration of this scale. 
These possibilities, in combination with the minimal evidence for a six factor solution provided by the a priori criteria for determining factors in this study, led the researchers to remove Factors Five and Six from subsequent analyses on the measure.

Results from the EFA provided evidence for the existence of either a two or four factor solution. All four factors have items that match the conceptualization of the POHLS measure, have eigenvalues above 1, and have at least three items that load onto that factor. However, two of the four factors (i.e., Factor Three; Lack of Motivation and Factor Four: Lack of Ability) have lower than desirable variance accounted for values. Additionally, as previously noted, the items from Factor Three and Factor Four are recoded items that were originally intended to load with the positively worded items from Factor One and Factor Two. This could also be the result of a wording bias of recoded items. This possibility was further explored in the analyses for Hypothesis One below. The items loading on these final four factors as determined by the collective results of the EFA, as well as their respective factor loadings, are presented in Table 6.

Hypothesis one predicted that the measurement model of the revised POHLS would provide a good fit to the data. As discussed previously, the results of the exploratory factor analysis conducted on the initial item pool provided evidence of either a two or a four factor solution. However, two of these factors (i.e., Factor Three: Lack of Motivation and Factor Four: Lack of Ability) likely reflect a negative wording bias, as they represent the absence of motivation and ability comprising Factors One and Two, respectively. As such, two measurement models were subjected to a confirmatory factor analysis. The first model consisted of all four factors as separate but correlated latent variables. Results of a confirmatory factor analysis indicated that the model provided a 
Table 6

Factor Loadings for Four Factor Solution

\begin{tabular}{|c|c|c|c|c|}
\hline Item & $\begin{array}{c}\text { Factor } \\
1\end{array}$ & $\begin{array}{l}\text { Factor } \\
2\end{array}$ & $\begin{array}{c}\text { Factor } \\
3\end{array}$ & $\begin{array}{c}\text { Factor } \\
4\end{array}$ \\
\hline 14. I have a hard time using suggestions I hear on how to live a healthy lifestyle. & .42 & .34 & .25 & .66 \\
\hline $\begin{array}{l}\text { 16. I am not able to figure out how to use the instructions a healthcare provider gives me once I } \\
\text { am home. }\end{array}$ & .26 & .50 & .37 & .70 \\
\hline 22. I do not use the health suggestions others give me to improve my health. & .33 & .36 & .30 & .58 \\
\hline 32. I am motivated to use information that I receive to improve my health. & .64 & .49 & .27 & .31 \\
\hline 34. I understand articles I see on how to be healthy. & .32 & .59 & .24 & .31 \\
\hline 38. I understand most of the words people use when they talk about health. & .43 & .65 & .35 & .38 \\
\hline $\begin{array}{l}\text { 40. When healthcare providers tell me how to treat myself at home (e.g., take medications), I } \\
\text { understand how to do so. }\end{array}$ & .42 & .71 & .42 & .46 \\
\hline 41. I am motivated to seek information which will improve my health. & .70 & .47 & .26 & .39 \\
\hline 50. I am able to understand discussions about health and healthy living. & .48 & .71 & .37 & .40 \\
\hline 51. I know how to seek information from others on how to improve my health. & .40 & 69 & .40 & .30 \\
\hline $\begin{array}{l}\text { 52. When I talk to a healthcare provider about my health, I am able to understand how I can } \\
\text { improve my health. }\end{array}$ & .40 & .72 & .35 & .39 \\
\hline $\begin{array}{l}\text { 54. When I talk to a healthcare provider about my health, I am able to understand how to } \\
\text { maintain good health. }\end{array}$ & .49 & 69 & .29 & .31 \\
\hline $\begin{array}{l}\text { 60. I am okay with not understanding everything a healthcare provider is talking about regarding } \\
\text { my health. }\end{array}$ & .23 & .31 & .62 & .27 \\
\hline 64. I am motivated to understand information that will help me live a healthy life. & .67 & .42 & .27 & .27 \\
\hline
\end{tabular}


65. I seek information which helps me maintain a healthy lifestyle.

66. It is okay with me to not understand all of the words a healthcare provider uses during my

visit.

67. I don't need to understand all of the words a healthcare provider uses during my visit.

$20 \quad .35$

.32

$32 \quad .22$

71. I am interested in understanding how to maintain a healthy lifestyle.

$.30 \quad .44$

$.75 \quad .44$

72. I seek information to help me improve my health.

.77

.40 
good fit to the data, $\chi^{2}(295)=612.95, p<.001, \mathrm{CMIN} / \mathrm{DF}=2.01, \mathrm{SRMR}=.07$, RMSEA $=.07$. The second model consisted of two latent variables. The first latent variable consisted of Factor One- Motivation and Factor Three-Lack of Motivation factors loading onto one latent variable, and the second latent variable was comprised of Factor Two- Ability and Factor Four- Lack of Ability. As it is a possibility that Factors Three and Four represent a negative wording bias, these items were loaded onto their respective positively worded latent variables- motivation and ability- and their error terms were correlated to account for this wording bias, a procedure that has been used with success in established measurements with evidence of similar wording biases (Beatty, PascualFerra, \& Levine, 2013; Goodboy, Martin, \& Bolkan, 2009). Results of a confirmatory factor analysis indicated that this two factor model also provided a good fit to the data, $\chi^{2}$ $(291)=617.43, p<.001, \mathrm{CMIN} / \mathrm{DF}=2.12, \mathrm{SRMR}=.08, \mathrm{RMSEA}=.08$. The four factor model can be found in Figure 3; the two factor model can be found in Figure 4. The standardized regression weights for items in the four factor and two factor models can be found in Tables 7 and 8, respectively.

Notably, the use of the SRMR and RMSEA values as a dual index to provide evidence of model fit is most appropriate when attempting to fit data to complex models (Hu \& Bentler, 1999). Baseline fit indices such as the Normed Fit Index (NFI; Bentler \& Bonnet, 1980), Incremental Fit Index (IFI; Bollen, 1989), and Comparative Fit Index (CFI; Bentler, 1990) penalize model complexity (Byrne, 2001; Hu \& Bentler, 1999). Given the number of parameters (i.e., a degree of freedom of 295) used in the models created to confirm the factor structure of the revised POHLS, using Hu and Bentler's (1999) dual index strategy was more appropriate in this context. 
Figure 3

\section{Four Factor Measurement Model}

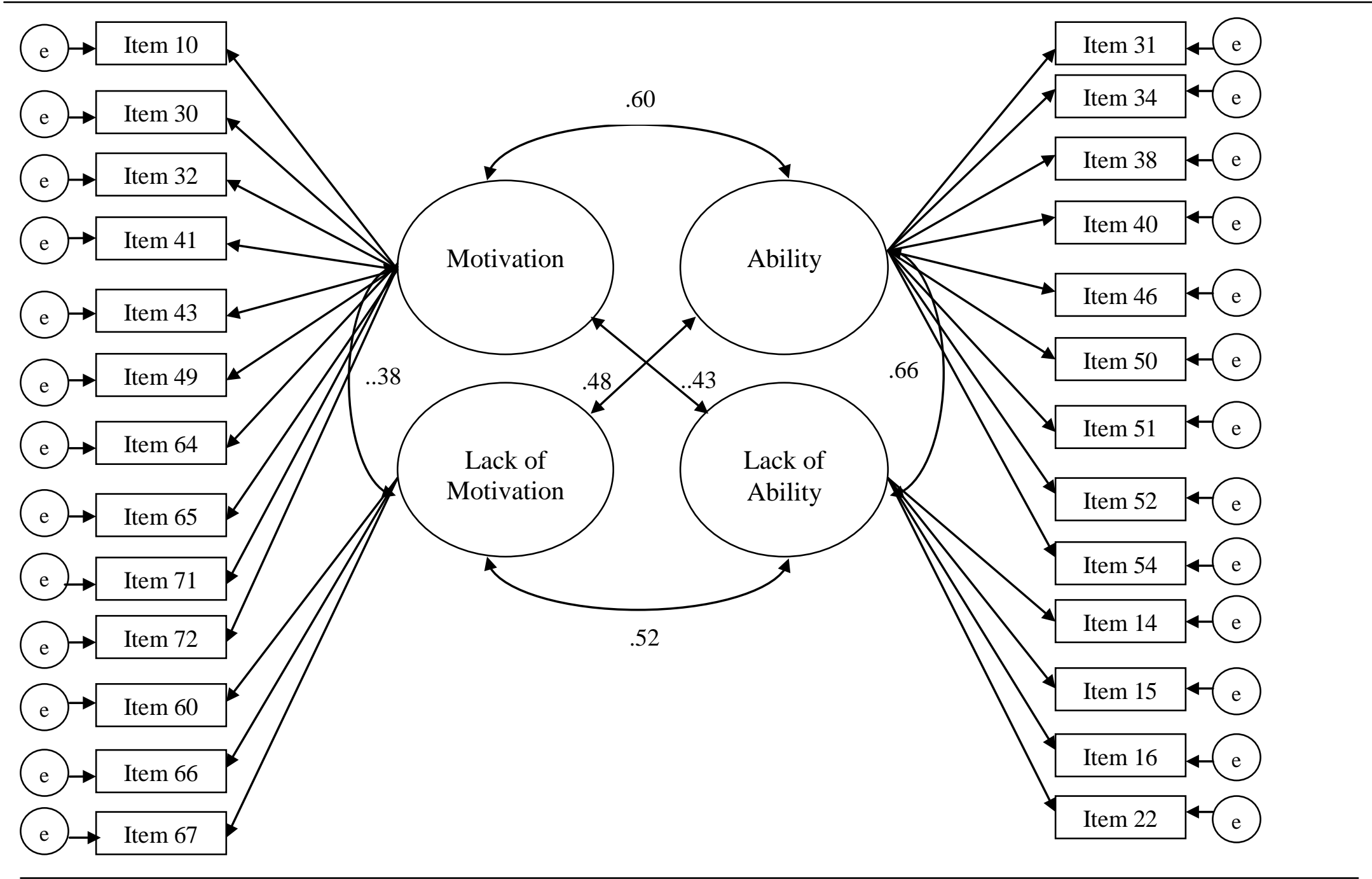


Table 7

Standardized Regression Weights for Four Factor Model

\begin{tabular}{|c|c|c|}
\hline Item & Factor & Estimate \\
\hline 10. & Motivation & .63 \\
\hline 30. & Motivation & .68 \\
\hline 32. & Motivation & .71 \\
\hline 41. & Motivation & .71 \\
\hline 43. & Motivation & .71 \\
\hline 49. & Motivation & .81 \\
\hline 64. & Motivation & .80 \\
\hline 65. & Motivation & .74 \\
\hline 71. & Motivation & .72 \\
\hline 72. & Motivation & .77 \\
\hline 60. & Lack of Motivation & .63 \\
\hline 66. & Lack of Motivation & .92 \\
\hline 67. & Lack of Motivation & .85 \\
\hline 31. & Ability & .59 \\
\hline 34. & Ability & .62 \\
\hline 38. & Ability & .63 \\
\hline 40. & Ability & .67 \\
\hline 46. & Ability & .60 \\
\hline 50. & Ability & .76 \\
\hline 51. & Ability & .64 \\
\hline 52. & Ability & .73 \\
\hline 54. & Ability & .78 \\
\hline 14. & Lack of Ability & .46 \\
\hline 15. & Lack of Ability & .43 \\
\hline 16. & Lack of Ability & .70 \\
\hline 22. & Lack of Ability & .49 \\
\hline
\end{tabular}


Figure 4

Two Factor Measurement Model

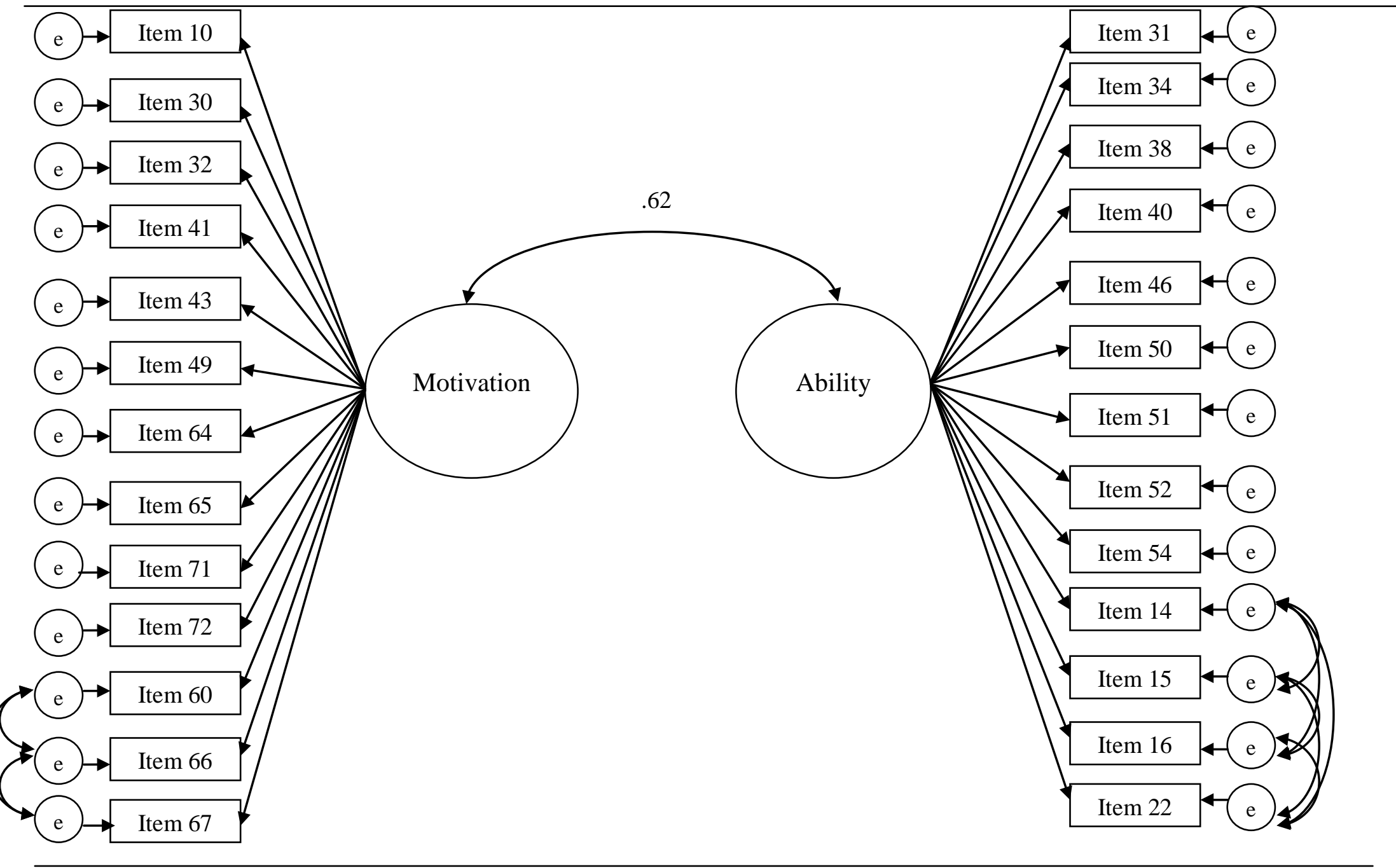


Table 8

Standardized Regression Weights for Two Factor Model

\begin{tabular}{|c|c|c|}
\hline Item & Factor & Estimate \\
\hline 10. & Motivation & .62 \\
\hline 30. & Motivation & .68 \\
\hline 32. & Motivation & .71 \\
\hline 41. & Motivation & .71 \\
\hline 43. & Motivation & .71 \\
\hline 49. & Motivation & .81 \\
\hline 64. & Motivation & .80 \\
\hline 65. & Motivation & .74 \\
\hline 71. & Motivation & .72 \\
\hline 72. & Motivation & .77 \\
\hline 60. & Motivation & .25 \\
\hline 66. & Motivation & .38 \\
\hline 67. & Motivation & .33 \\
\hline 31. & Ability & .60 \\
\hline 34. & Ability & .62 \\
\hline 38. & Ability & .63 \\
\hline 40. & Ability & .67 \\
\hline 46. & Ability & .60 \\
\hline 50. & Ability & .76 \\
\hline 51. & Ability & .63 \\
\hline 52. & Ability & .73 \\
\hline 54. & Ability & .78 \\
\hline 14. & Ability & .30 \\
\hline 15. & Ability & .16 \\
\hline 16. & Ability & .49 \\
\hline 22. & Ability & .39 \\
\hline
\end{tabular}


As both the four factor and two factor models provided a good fit to the data, the researchers turned to the conceptualization and theory underlying the factor analysis procedures in order to determine which structure best represented the nature of the revised POHLS. As stated previously, the four factor model presents the latent variables of Motivation, Ability, Lack of Motivation, and Lack of Ability. The two factor model presents a first factor of Motivation, which consists of both positively and negatively worded items assessing an individual's motivation to gain access to, understand, and use health information, and a second factor of Ability, which similarly consists of both positively and negatively worded items reflecting an individual's ability to engage in these tasks. For both conceptual and practical reasons, moving forward with the two factor version of the measurement model is preferable. In order to select one of two models that provide evidence of good fit, however, it was first necessary to establish that the differences in fit between the two models was not significant. As such, a Chi Square difference test comparing the ratio of the model Chi Square to its degree of freedom for both the two and four factor models was conducted. Results of this difference test indicated that the Chi Square to degree of freedom ratios for these two models were not significantly different, $\chi^{2}(4)=4.48, p=.34$. As the two and four factor models were therefore mathematically equal, the decision was made to proceed with the two factor model for all subsequent analyses included in this manuscript. The first hypothesis was supported, and the revised POHLS was finalized as a two factor 26-item measure. The final measure is included in Appendix K.

Hypothesis two predicted that health literacy would be positively correlated to perceived health competence, but at a level no higher than .70. Results of a Pearson 
product-moment correlation using a university student sample indicate that perceived health competence was positively correlated with both the motivation $(r=.28, p<.001)$ and ability $(r=.37, p<.001)$ factors of the revised POHLS. Further, neither correlation exceeded .70. Hypothesis two was supported.

Hypothesis three predicted that health literacy would be positively correlated to patient efficacy, but at a level no higher than .70. Results of a Pearson product-moment correlation using a university student sample indicate that patient self efficacy was positively correlated with both the motivation $(r=.38, p<.001)$ and ability $(r=.60, p$ $<.001$ ) factors of the revised POHLS. Patient response efficacy was also positively correlated with both the motivation $(r=.45, p<.001)$ and ability $(r=.51, p<.001)$ factors of the revised POHLS. Further, none of these correlations exceeded .70. Hypothesis three was supported.

Hypothesis four predicted that health literacy would be negatively correlated to communication anxiety, but at a level no higher than .70. Results of Pearson productmoment correlation using a university student sample indicate that trait communication anxiety was not related to the motivation factor $(r=-.09, p=.21)$ of the revised POHLS, but was negatively related to the ability factor $(r=-.21, p<.01)$. Dyadic communication anxiety was negatively correlated with both the motivation $(r=.-.31, p<.001)$ and ability $(r=-.32, p<.001)$ factors of the revised POHLS. Small group communication anxiety was negatively correlated with ability $(r=-.17 p<.05)$ but not motivation $(r=.02, p=$ .81). Public speaking communication anxiety was not significantly correlated to either the motivation $(r=.01, p=.87)$ nor ability $(r=-.08, p=.27)$ factors of the revised POHLS. Hypothesis four was partially supported. A correlation matrix of all variables 
Table 9

Correlations Between Health Literacy Factors, Perceived Health Competence, Patient Efficacy, and Communication Anxiety

\begin{tabular}{|c|c|c|c|c|c|c|c|c|}
\hline Variable & 1 & 2 & 3 & 4 & 5 & 6 & 7 & 8 \\
\hline 1. Motivation & -- & & & & & & & \\
\hline 2. Ability & $.63 * *$ & -- & & & & & & \\
\hline 3. Perceived Health Competence & $.28 * *$ & $.37 * *$ & -- & & & & & \\
\hline 4. Patient Self Efficacy & $.38 * *$ & $.60 * *$ & $.30 * *$ & -- & & & & \\
\hline 5. Patient Response Efficacy & $.45 * *$ & $.51 * *$ & .07 & $.40 * *$ & -- & & & \\
\hline 6. Trait Communication Anxiety & -.09 & $-.21 *$ & $-.17^{*}$ & $-.28 * *$ & .05 & -- & & \\
\hline 7. Dyadic Communication Anxiety & $-.31 * *$ & $-.32 * *$ & $-.15^{*}$ & $-.29 * *$ & -.13 & $.73 * *$ & -- & \\
\hline $\begin{array}{l}\text { 8. Small Group Communication } \\
\text { Anxiety }\end{array}$ & .02 & $-.17^{* *}$ & $-.16^{*}$ & $-.23 * *$ & .04 & $.90 * *$ & $.51^{* *}$ & -- \\
\hline $\begin{array}{l}\text { 9. Public Speaking Communication } \\
\text { Anxiety }\end{array}$ & .01 & -.08 & -.14 & $-.20 * *$ & .13 & $.87 * *$ & $.40^{* *}$ & $.71^{* *}$ \\
\hline
\end{tabular}

Note. ${ }^{*} p<.05 . * * p<.001$. 
included in Hypotheses Two, Three, and Four is provided in Table 9.

Hypothesis five predicted that university students who have completed a course in Health Communication would have higher health literacy levels than students who have not completed a course in Health Communication. Results of an Independent Samples ttest indicate that there was a significant difference between these groups for both motivation $(t(57)=-3.46, p=.001)$ and ability $(t(57)=-3.55, p=.001)$. Students who had completed the Health Communication course had higher motivation $(M=55.64, S D$ $=6.19)$ and ability $(M=55.00, S D=5.46)$ than students who had not completed a course in Health Communication (Motivation, $M=49.47$, $S D=7.16$; Ability, $M=50.03, S D=$ 5.20). Hypothesis five was supported.

Hypothesis six predicted that health literacy would be negatively correlated with physiological indicators of risk for diabetes and obesity. Results of a Pearson productmoment correlation using a community adult sample indicate that neither motivation $(r=$ $.02, p=.89)$ or ability $(r=-.07, p=.70)$ was significantly correlated to blood glucose levels. Further, results of a Pearson product-moment correlation using a community adult sample indicate that neither motivation $(r=.00, p=.99)$ nor ability $(r=-.13, p=.45)$ was significantly correlated to Body Mass Index. Hypothesis six was not supported.

Hypothesis seven predicted that individuals’ health literacy levels would be able to effectively predict their placement in the stages of readiness for change regarding physical activity and healthy eating. Using a community adult sample, two direct discriminant analyses were conducted with two predictors of membership in five groups. In the first discriminant analysis, the two predictors were the motivation and ability subscales of the revised POHLS; the five groups were the stages of change according to 
the Transtheoretical Model (i.e., Precomtemplation, Contemplation, Preparation, Action, and Maintenance) regarding individuals' intentions toward physical activity. Results of this analysis indicated that motivation and ability were not able to predict an individual's placement in the stages of change for physical activity, as the two discriminant functions derived yielded a combined $F(8,3)=.83, p=.59$.

In the second discriminant analysis, the two predictors were the motivation and ability subscales of the revised POHLS; the five groups were the stages of change according to the Transtheoretical Model (i.e., Precomtemplation, Contemplation, Preparation, Action, and Maintenance) regarding individuals' intentions toward healthy eating. Results of this analysis indicate that motivation and ability were not able to predict an individual's placement in the stages of change for healthy eating, as the two discriminant functions derived yielded a combined $F(8,3)=.68, p=.09$.

Hypothesis eight predicted that individuals exposed to a brief educational intervention on health literacy would have higher change scores in their health literacy levels that individuals not exposed to the intervention. Following the methodology suggested by Kerlinger (1986) to examine the difference in groups from pretest to posttest in experimental design, results of an Independent samples t-test using a university student sample indicate that there was not a significant difference in the change scores between these groups for either motivation $(t(202)=-.44, p=.66)$ nor ability $(t(203)=-.42, p=.67)$. The motivation and ability scores (i.e., mean, standard deviation, and change scores) of the experimental and control groups are provided in Table 10. Hypothesis eight was not supported. 
Table 10

Motivation and Ability Scores for Experimental and Control Group at Pretest and Posttest

\begin{tabular}{lll}
\hline & Experimental Group & \\
& & Control Group $^{\mathrm{b}}$ \\
Pretest Motivation & $50.51(7.57)$ & $48.89(7.92)$ \\
Posttest Motivation & $50.88(8.16)$ & $48.86(7.86)$ \\
Change Score Motivation & .37 & -.03 \\
Pretest Ability & $51.74(6.25)$ & $51.32(6.73)$ \\
Posttest Ability & $50.56(7.94)$ & $49.77(6.82)$ \\
Change Score Ability & -1.18 & -1.55 \\
\hline
\end{tabular}

Note. ${ }^{\mathrm{a}} \mathrm{N}=139{ }^{\mathrm{b}} \mathrm{N}=65$. 


\section{Summary of Chapter III}

This chapter presented the findings from the sole research question and eight hypotheses of this dissertation. The results of the analyses employed in this chapter indicate that the Perceived Oral Health Literacy Scale (POHLS; LaBelle \& Weber, 2013) was successfully revised to more accurately reflect the World Health Organization (WHO, 2014) definition of health literacy. The results of the exploratory factor analysis on the new item pool argued for the existence of two discrete yet related dimensions: Motivation and Ability. Results of the confirmatory factor analysis conducted on a separate sample supported these findings. The revised POHLS consists of 26 items (i. e, 13 items on both the Motivation and Ability dimensions) which assess individuals' motivation and ability to gain access to, understand, and use health information from various sources in order to promote and maintain good health.

Following the revision of the POHLS, the newly created scale was subjected to a number of tests in order to assess its reliability and validity. The measure performed admirably in terms of reliability across three additional samples $(\alpha=.89-.91$ for the Motivation factor; $\alpha=.84-.88$ for the Ability factor). Additionally, the positive relationships obtained with perceived health competence and patient self- and response efficacy provide further evidence for the measures construct validity. Notably, none of these correlations exceeded the a priori effect size level of .70, alleviating concerns of isomorphism among these variables. Ability was also negatively correlated to individuals' trait, dyadic, and small group communication anxiety; Motivation was negatively correlated with dyadic communication anxiety. Further evidence of construct validity was provided with a known groups validity assessment, as individual's 
participating in a semester-long course on Health Communication had higher scores on both the Motivation and Ability subscales than individuals who had not participated in the course.

An attempt to establish the criterion-related validity of the measure was not supported, as an individual's score on neither the Motivation nor the Ability subscale could predict his or her physiological indicators of risk for diabetes and obesity (i.e., blood glucose levels and Body Mass Index). Further, an individuals’ Motivation and Ability per the revised POHLS could not effectively place him or her into one of the five stages of change for physical activity and healthy eating behaviors.

Finally, the results of a brief educational intervention conducted on health literacy were provided. The outcome of a pretest-posttest nonequivalent groups design did not provide support that health literacy is a construct that can be influenced through such efforts. 


\section{CHAPTER IV}

\section{Discussion}

There were two purposes of this dissertation. The first purpose was to develop a valid and reliable measurement of health literacy appropriate for use in the social sciences. The construction of this measurement was based upon the World Health Organization (WHO, 2014) definition of the construct, which states that health literacy is "the cognitive and social skills which determine the motivation and ability of individuals to gain access to, understand and use information in ways which promote and maintain good health” (“Track 2: Health literacy and health behaviour,” para. 1). The second purpose was to determine if health literacy can be increased through the intervention efforts of communication studies scholars. To address these two purposes, this chapter begins with a collective discussion of the research question and eight hypotheses of this dissertation, followed by the implications of the findings, the limitations of the studies, and future directions for research.

\section{Scale Development}

Addressing the first purpose of this dissertation involved revising the Perceived Oral Health Literacy Scale (POHLS; LaBelle \& Weber, 2013). The original POHLS is a ten-item scale that assessed individuals' health literacy via three factors: Question Asking, Adequacy of Information, and Ability to Apply Healthcare Directives. Although this original measure was reliable and offered an important first step in designing a measure of health literacy suitable for use by social science researchers, there was concern that the items and corresponding factors might reflect satisfaction with a healthcare provider more than an individuals' health literacy. To address this potential issue, an additional 54 
items were developed that more directly assessed one's motivation and ability to gain access to, understand, and use health information. These items, along with the original item pool for the POHLS, were subjected to an exploratory factor analysis using a university student sample to answer the first and only research question of this dissertation.

The factor structure observed in this analysis was then tested and validated in a second university student sample through the use of confirmatory factor analytic methods. The results of these analyses provided evidence that the optimal solution for the revised POHLS is a two factor solution which assesses individual's Motivation and Ability to gain access to, understand, and successfully use health information from a variety of sources. Importantly, the representativeness of these factors to the definition of health literacy used in this study provides evidence of the content validity of the revised POHLS (Kerlinger, 1986). The content validity of a measure is determined by assessing if the substance of the measure is representative of the content that it is intended to measure (Kerlinger, 1986). The items of the revised POHLS create factors which are consistent with the WHO (2014) definition of the construct and the individual items within each factor include references to gaining access to, understanding, and using health information.

The emergence of motivation and ability as factors in the revised POHLS is also very consistent with the vast body of research on human behavior in the context of persuasion and health. Widely used theories such as the Health Beliefs Model (Rosenstock, 1966), Protection Motivation Theory (Rogers, 1975), the Theory of Reasoned Action (Fishbein \& Ajzen, 1975), the Theory of Planned Behavior (Ajzen, 
1991),the Extended Parallel Processing Model (Witte, 1992), and the Risk Perception Attitude Framework (Rimal \& Real, 2003) emphasize that an individual's sense of susceptibility to a threat and ability to manage that threat are important antecedents to his or her subsequent behavior. The widely used dual process theories of persuasion, the Elaboration Likelihood Model (ELM; Petty \& Cacioppo, 1986) and the Heuristic Systematic Model (HSM; Eagly \& Chaiken, 1993) forward that individuals’ motivation and ability regarding a given topic determine how they will process a message, which has a significant impact on ultimate altitudinal and behavioral outcomes. It would behoove future researchers of health literacy, especially those using the revised POHLS, to apply what is already known regarding the antecedents and outcomes of motivation and ability in the context of persuasion to inform future investigations. How health literacy adds to the research literature on health decision making and message processing is yet to be discovered.

Importantly, the factors that emerged in the revision of the POHLS also address many of the calls for an improved measure of health literacy. Specifically, the inclusion of the Motivation factor in the revised scale speaks to what Nutbeam (2000) referred to as “critical literacy” (p.6). That is, by conveying that the motivation to gain access to, understand, and use health information is just as important as the ability to perform these tasks, the revised POHLS implies that health literacy is much more than a set of basic functional skills such as reading written text and interpreting numbers. Rather, a critical approach to studying health literacy acknowledges the individual consciousness that is key to increasing health literacy levels, and how educational efforts to improve this skill set must acknowledge individual motivation and interest as well. 
Aside from addressing motivation, the revised POHLS also acknowledges the wide variety of sources and quality of health information in the modern world. As stated by Mackert, Champlin, Pasch, and Weiss (2013), existing measures of health literacy have not been able to distinguish between people who struggle to find relevant health information and those who have difficulty understanding and applying that information once they have acquired it. Recognizing the difference between having difficulty finding information and having difficulty understanding that information is crucial to moving forward in studying health literacy in the modern health context, where individuals are exposed to such a vast amount of health information from various sources (Mackert et al., 2013). Whether the source of the information is a nutrition label, a commercial for a pharmaceutical drug, an article found from an online source, or a discussion with family and friends, it is important to account for individual's ability to discern information that is accurate and useful from that which is not. As noted by Mackert and colleagues (2013) it is time for scholars who study health literacy to consider if what have previously been assessed using health literacy measures (i.e., comprehension) is as important at individuals' ability to find important and useful information. The inclusion of items which address this ability is a distinct strength of the measure developed in this study. The revised POHLS has a distinct focus on motivation and ability to gain access to health information as well as understanding it.

Finally, the revised POHLS is perhaps the first measure of health literacy to address the distinctly communicative or interactive nature of the health literacy phenomenon (Nutbeam, 2000). Unlike the REALM, the TOFLA, or the NVS, the revised POHLS is self-administered, applies to health literacy across a wide range of 
contexts and information searches (namely, beyond the healthcare provider-patient interaction or the reading of nutrition labels), and accounts for more than the cognitive or numerical skills associated with health literacy. As stated in Chapter I, health literacy encompasses much more than reading ability, numeracy, or computer literacy. These cognitive skills, although certainly an important part of an individuals’ overall health literacy, are not the sole driving factor in how well individuals perform in the health environment. Rather, as noted by the WHO definition of the construct (2014), health literacy is comprised of both cognitive and social skills. The revised POHLS presented in this dissertation addresses individuals' social skills in obtaining information, in communication with others about health, and in understanding discussions related to their own health. This will allow future researchers to examine how the social skills that are a part of health literacy function in a wide variety of contexts. Additionally, this measure answers Baker’s (2006) call for a self-report measure of health literacy that addresses comprehension of spoken health-related information. In developing a self-report measure of this kind, this dissertation offers an assessment of health literacy that can be taken outside of the clinical context and applied to much wider variety of scholarship and application.

The construct and criterion-related validity of the revised POHLS were then addressed through six hypotheses (i.e., Hypotheses two through seven). Specifically the measure's construct validity was addressed in tests of its convergent, discriminate, and known-groups validity. The criterion-related validity of the revised POHLS was assessed in tests of predictive validity. 
Validity assessments. The convergent and discriminate validity of the revised POHLS were determined by examining the measure's relationship to three constructs: perceived health competence, patient self and response efficacy, and communication anxiety. As discussed in Chapter I, these variables should theoretically be associated with individuals' motivation and ability to gain access to, understand, and use health information from healthcare providers, family and friends, and other sources. Perceived health competence is defined as the degree to which an individual feels capable of effectively managing his or her health outcomes (Smith et al., 1995) and has been positively correlated with overall physical and psychological well-being and health, sense of control over health outcomes, and both intention and enactment of positive health behaviors (Smith et al., 1995). Patient self efficacy comprises one's perception that he or she can perform the recommended behaviors of a healthcare provider; patient response efficacy reflects the degree of confidence he or she has that those behaviors will be effective in improving health outcomes (Witte, 1992; Young \& Klingle, 1996). Finally, communication anxiety involves "the fear or anxiety associated with real or anticipated communication with others” (Booth-Butterfield, 2008; Booth-Butterfield \& Gould, 1986; McCroskey, 1977) and has been associated with a number of health outcomes (Ayres et al., 1996; Richmond et al., 1998; Wheeless, 1984).

Results using a university student sample indicate that both an individuals’ motivation and ability regarding health literacy were positively correlated to perceived health competence, patient self and response efficacy, and negatively correlated to dyadic communication anxiety. Further, one’s ability to gain access to, understand, and use health information is negatively related to his or her trait communication anxiety across 
contexts and to anxiety in the small group context. Neither motivation nor ability is related to communication anxiety in the public speaking context. The relationship of the factors of the revised POHLS to theoretically related constructs offers much support for the convergent validity of the scale; that is, the measure is related to variables it should theoretically be related to (Kerlinger, 1986). Equally important in developing a new measure, the tests of the hypotheses also provided support for the notion that the revised POHLS is assessing a distinct construct through establishing its discriminate validity (Kerlinger, 1986). That is, the revised POHLS does not appear to be isomorphic with measures of perceived health competence, patient self or response efficacy, and communication anxiety. Previous researchers have argued that construct isomorphism might be a concern when correlation coefficients between measures reach between .70 and .90 (Barrett, 1986; Fischer, Vauclair, Fontaine \& Schwartz, 2010; Ten Berge, 1986; Weber \& Patterson, 2000). Since the correlations observed between the constructs in the present study failed to reach these levels, the results argue against isomorphism and for the uniqueness of the POHLS measure.

Although the lack of a significant relationship to communication anxiety in the public speaking context did not allow for full support of the fourth hypothesis, the results conceptually make sense. An individual's health literacy should be associated with how well he or she could communicate in dyadic contexts such as interactions about health with a healthcare provider, nurse, insurance provider, and life partners/spouses. These health-related skills however, would rarely be enacted in front of a large group of individuals (re: public speaking). The lack of association actually offers further support 
for the discriminant validity of the scale in that the factors Motivation and Ability were not related to a communication context that theoretically they should not be related to.

The lack of association between an individual's motivation to gain access to, understand, and use health information with his or her trait communication anxiety and anxiety in the small group context is also conceptually clear. The ability dimension of the revised POHLS concerns whether one can understand health information from various sources (e.g., healthcare providers, articles, discussions with others). Specifically, there are items on this factor which mention "discussions about health and healthy living," and seeking information from "others” on how to improve health outcomes, and understanding when "people” give suggestions on how to be healthy. Many of these items therefore imply group discussions, perhaps with family and friends, on health-related information. This is in contrast to the items on the motivation factor, which are more self-controlled and do not focus as heavily on group discussions (e.g., "I am motivated to understand information that will help me live a healthy life” and "I seek information to help me improve my health"). In fact, discussions with other persons on the motivation factor are almost entirely about the healthcare provider (e.g., "I am okay with not understanding everything a healthcare provider is talking about regarding my health”), which explains the relationship this factor had with dyadic communication anxiety. However, responses on this factor should not necessarily be related to small group discussions or even trait communication anxiety. It makes more sense for the ability factor, which encompasses interactions with both healthcare providers and small groups, to be related to overall assessment of trait communication anxiety than its motivation counterpart. 
It is also likely that one could be trait communication anxious but very motivated to gain access to, understand, and use health information. The issues related to communication anxiety would arise not in being motivated to seek health information from various sources, but in actually being able to do so. That is, a person with high trait communication anxiety could be highly interested in seeking information from various sources in order to improve his or her health (i.e., motivation), but feel impeded in actually doing so (i.e.: ability). Therefore it would follow that one's motivation related to health literacy would not necessarily be related to his or her trait communication anxiety, although this trait is likely related to his or her ability to seek, understand, and apply health information.

A third assessment of construct validity was provided in a test of the knowngroups validity of the revised POHLS. Results indicate that undergraduate university students who participated in a 16 week course on Health Communication had higher motivation and ability than students who had taken a non-health related course. The ability of a measure to differentiate one theoretically distinct group from another is a key aspect of establishing not only its validity but its utility to future researchers of the construct (DeVellis, 2012; Eagly \& Chaiken, 1993). Additionally, the results of this hypothesis (i.e., five) offer support for why there is a nation-wide growing interest and expansion of Health Communication courses and programs (Sharf, 1999); Health Communication students are effectively increasing the communication skill sets that they need to succeed in the modern health environment by taking these courses.

The criterion-related validity of the revised POHLS was addressed in hypotheses six and seven, which assessed the predictive validity of the new measure in a specific 
health context and using a community adult sample. As mentioned in Chapter I, predictive validity assessments are judged by how well a measure can predict an outside criterion and by checking the relationship of the measurement to some present or future outcome (Kerlinger, 1986). As this type of validity is usually determined for practical application (DeVellis, 2012), the revised POHLS was examined for its utility in an applied health context: the physiological indicators of risk for obesity and diabetes in an at-risk population. This validity assessment also incorporated a widely used theory of individual decision making, the Transtheoretical Model (TTM; Prochaska \& DiClemente, 1983). The results of these hypotheses collectively did not provide support for the predictive validity of the revised POHLS. An individuals' motivation and ability per the measure did not predict his or her blood glucose level, body mass index, or his or her placement in the stages of change of the TTM.

In reflecting upon the failure in establishing the criterion-related validity of the revised POHLS, a crucial factor appears to drive the lack of significant results. Notably, hypotheses six and seven were tested using a sample of adult visitors to a community center in North Central West Virginia. This sample was intentionally selected, as West Virginians have a high prevalence of obesity and diabetes (CDC, 2011a; HSC, 2012). However, the sample derived for this study appears to have not been an adequate representation of the average health of a West Virginia resident. The average blood glucose level obtained in this sample was $90.73 \mathrm{mg} / \mathrm{dL}$; per the blood glucose monitoring system used in this study, this is well within the normal range of blood glucose levels. In fact, one is not considered to by hyperglycemic until their blood glucose level is above $240 \mathrm{mg} / \mathrm{dL}$. Further, the range of blood glucose levels obtained in this study was only 
from a minimum reading of $74 \mathrm{mg} / \mathrm{dL}$ to $128 \mathrm{mg} / \mathrm{dL}$. The lack of variability in the blood glucose measures of participants does not allow for an accurate assessment of its association with the revised POHLS.

Although the Body Mass Indices (BMI) of participants had slightly more variability, with a range in BMI from 19.96 - 49.00, the majority of participants $(n=14$, 38.9\%) were in a normal weight range. Further, most participants indicated that they currently eat a diet of low fat and low calorie foods (i.e., $n=14,35 \%$ ) were in the Maintenance stage regarding healthy eating. The relatively healthy status of this sample, in combination with the lack of variability in the blood glucose and BMI assessments, might have prevented a statistically significant relationship from emerging between these indicators of health and the factors of the revised POHLS.

There is, of course, the possibility that the revised POHLS is not directly related to any of the above constructs. Osborn and colleagues (2010) found that health literacy, as indicated by results of the Rapid Estimate of Adult Literacy in Medicine (REALM; Davis et al., 1993), was not related to an assessment of blood glucose using an A1C test. Rather, the diabetes self efficacy of participants mediated this relationship. Furthermore, the model obtained by the researchers included variables such as age, race, diabetes diagnosis, and year of insulin use to predict A1C with success. As the study reported here only assessed the association of the revised POHLS to a measure of blood glucose levels, it is quite possible that the lack of statistical significance could be attributed to not examining important mediators and moderators of this relationship. As noted by Baker (2006), the eventual health outcomes associated with health literacy are affected by a host of individual, situational, and system-wide factors. Perhaps future studies employing the 
revised POHLS could establish its predictive validity using blood glucose level assessments and BMI as outcomes by taking these factors into account.

In sum, the results reported here provide strong arguments for the content and construct validity of the POHLS measure among university student respondents. The two factors and corresponding items which comprise the measure, Motivation and Ability, adequately represent an individuals' cognitive and social skill set needed to gain access to, understand, and use health information. The construct validity of the scale was evidenced by in its convergent and discriminate associations with perceived health competence, patient self and response efficacy, and communication anxiety. Further evidence of the construct validity of the measure was provided in its ability to differentiate between theoretically distinct groups (i.e., known groups validity). Although there was not support for the criterion-related validity of the measure, this was potentially due to sampling issues. Overall, the scale development and reliability and validity assessments outlined in this section answered the first purpose of this dissertation, to establish a valid and reliable measure of health literacy for use in social science research. The ways in which the second purpose of this dissertation, to determine if health literacy is a construct that can be increased through the intervention efforts of communication studies scholars, will be discussed next.

\section{Increasing Health Literacy}

The second purpose of this dissertation was to determine if health literacy can be increased through the intervention efforts of communication studies scholars. To address this purpose, a brief educational intervention was conducted in an undergraduate university course setting in which an experimental group took part in a lesson on 
improving cognitive and social skills related to health literacy, and a control group took part in a non-health related lesson. The results of this pretest-posttest nonequivalent groups design did not provide support for the notion that health literacy can be increased through educational efforts.

The failure of the intervention to impact individuals' motivation scores is disheartening, as developing interventions to increase health literacy related motivation is a specific focus of the U.S. Department of Health and Human Services (Berkman et al., 2011). In line with the suggestions of Nutbeam (2000), increasing individual's motivation to correctly process health information likely plays a significant role in their ability to do so. Although much of the intervention work on health literacy comprises efforts to enhance ability to comprehend health information, it is possible that creating materials which increase motivation is just as, if not more, important to eventual health outcomes (Berkman et al., 2011). Berkman and colleagues (2011) call upon health communication scholars to guide those who design health literacy interventions to create effective messages to increase motivation (e.g., Capella, 2006). Moving forward, future research should focus more specifically on how to increase individuals’ motivation to gain access to, understand, and use health information in ways which promote and maintain good health.

A possible explanation for why there was not a significant increase in the health literacy of the experimental group from pretest to posttest was the length of the educational intervention that was conducted. Although the intention of the intervention was always to be brief, logistic constraints (i.e., having to deliver a lesson on health literacy as well as distribute and collect the posttest questionnaire in a 50 minute class 
period) caused the lesson on health literacy to be only 35 minutes in length. This is in comparison to health education interventions that have taken up to one year to complete (Gerber et al., 2005). Further, the intervention was conducted using an undergraduate university student sample. As several researchers (Duncan et al., 2002; Fleming et al., 2010; Roberts \& Kennedy, 2006) have noted, young adults often have a feeling of invincibility in regard to their own health. It is therefore likely that the audience used for the intervention did not view health literacy as a personally relevant topic, which would have led to a decreased motivation to closely process the materials being presented (Petty \& Cacioppo, 1986). It is possible that an audience with a higher sense of personal relevance for the topic would have reacted to the message presented very differently. Future assessments of health literacy interventions should be mindful of using a university student sample in attempting to create effective health literacy messages.

It is also likely that extraneous variables play a role in the ability to increase health literacy with educational interventions. As can be seen in Appendix N, the primary focus of the intervention on health literacy was to give participants tips on how to interact with healthcare providers (e.g., what questions to make sure to ask), how to find quality health information from various sources, and where to go should a health problem arise. Yet, as Wilson and colleagues (2010) found in their intervention on the retention of information pertinent to colorectal screening among low health literacy patients, the role of cognition is incredibly important in predicting ability to process health information. Patients' health literacy, in fact, played a lesser role in their ability to recall information from a video about colorectal cancer than their processing speed, working memory, or long-term memory one week later. As the researchers assert, the 
most prominent approach to ameliorating low health literacy is to improve the readability and simplicity of written health materials, but future efforts should be made to increase cognitive skills such as working memory and processing speed. The design of the current investigation did not allow for the measurement or analysis of participants' cognitive skills in this manner; perhaps more direct attempts to improve these skills would have led to increases in the ability dimension for the experimental group.

Indeed, in their review of published articles on health literacy interventions, Berkman and colleagues (2011) found that while most interventions used a single strategy to increase health literacy (e.g., provide graphical presentations of material, enhance readability of written material) those interventions which combined multiple strategies had more success in increasing individuals' health outcomes. These results suggest that health literacy interventions should address multiple aspects of health literacy, perhaps more than was covered in the intervention materials of the current investigation, in order to be successful. As Berkman and colleagues (2011) note, however, there should be additional research on which specific components of these interventions are most successful. Multiple trials could be conducted on health literacy, for instance, in which the relative cost and effect of each component is determined. This would allow future researchers and practitioners to focus exclusively on the components that lead to successful health outcomes and eliminate those that do not have a significant impact.

Although the results of the educational intervention did not succeed in influencing participants' health literacy, the results presented in this dissertation do provide some evidence to support the argument that health literacy can be increased. Specifically, the 
results of the known-groups validity assessment indicate that individuals who participated in a sixteen week course on Health Communication had higher motivation and ability scores than individuals who completed concurrent courses in Business Communication and Advanced Interpersonal communication. As a course in Health Communication is essentially an “educational intervention” meant to increase individuals’ communication skills related to functioning successfully in the healthcare environment, the success of this validity assessment also suggests that attempts to increase motivation and ability related to health literacy can in fact succeed.

Important considerations should be taken into account if adapting the design of the known-groups assessment to an experimental design, such that the participants in the Health Communication course constitute an "experimental” group and the participants completing a course in either Advanced Interpersonal or Business Communication are the “control” group. As this would be a posttest only nonequivalent groups design, an obvious weakness is that the two groups are merely assumed to be equal in all other aspects than exposure to the manipulation of the independent variable (in this case, the subject of the course in which they are enrolled). Without randomization to each group, it cannot be assumed that the two groups were equal in terms of their relative motivation and ability previous to completing the course (Kerlinger, 1986). It is very likely, for instance, that individuals who are interested, motivated, and knowledgeable about health care and the health environment are more likely to enroll in a health communication course. As the health literacy of the university student sample used in the known-groups assessment was not obtained at the onset of the semester, it is not possible to establish a 
causal relationship between taking the course and increased health literacy using these results.

Yet, as Kerlinger (1986) notes, it is possible to examine pertinent characteristics of each group in order to make a relative assessment of their similarity. As evidenced in Table 4, the students in the Health Communication course were markedly similar to those in the non-Health Communication courses in terms of age, ethnicity, and class rank. The only demographic variable in which the groups differ noticeably is sex: the Health Communication course was comprised mostly of females, whereas the opposite was true for the non-Health Communication courses. Results of an Independent samples t-test for motivation, $t(57)=-.82, p=.41$, and ability, $t(57)=-.15, p=.88$, however, indicated that there were no sex differences in the health literacy scores of male and female participants overall. Thus, while there are admittedly weaknesses in relying solely on the results of the known-groups assessment to claim that health literacy is a construct that can be increased through educational efforts the results of this study certainly provide a reason to continue future research toward this purpose.

In sum, the second purpose of this dissertation was addressed through two pieces of evidence: the results of an educational intervention and the results of a known-groups assessment. Although the educational intervention was not successful in increasing individuals' motivation and ability in regard to health literacy, there are plausible reasons for this failure that should not be overlooked and be addressed in future research. The results of the known groups assessment provide an argument for conducting further research on increasing the health literacy skill set, as the health literacy of a group of individuals who had completed a lengthier educational course on Health Communication 
did have higher rates of motivation and ability than those who did not. As such, there is some evidence to suggest that health literacy is not a static or fixed construct, but rather one that is able to change and be influenced over time. This has important implications for future research and practical application.

\section{Implications}

The results of this dissertation have a number of implications for both scholars and healthcare practitioners contributing to the study and treatment of health literacy. The studies reported here offer a reliable and valid measure to assess health literacy for use in social science research. The contribution of a measure which assesses the communicative, interactive, and critical level of health literacy as opposed to simply basic functional skills was not only sorely needed in the literature (IOM, 2004; Nutbeam, 2000), it has the potential to make a noticeable impact on the approach to studying the construct (IOM, 2004; McCormack et al., 2013). Further, the revised POHLS offers an assessment of health literacy which spans across multiple contexts and health conditions; this represents a needed move in the field of health literacy toward broader measurement and conceptualization of the construct (McCormack et al., 2013). With a more widely accepted and easily accessible assessment of health literacy, researchers can begin to develop more comprehensive theoretical models to understand the complexity of the health literacy phenomenon (McCormack et al., 2013). Indeed, much of the literature on health literacy has been descriptive in nature; there are hundreds of studies on the outcomes associated with health literacy and its prevalence (see Berkman et al., 2011 for a review), with a much lesser focus on model development and efforts to explain and predict issues associated with health literacy (e.g., Baker, 2006). 
Future studies on health literacy using the revised POHLS should be mindful of the exact role of this measurement in examining the overall phenomenon of health literacy. This measurement focuses on assessing the cognitive and social skills which predict individual's motivation and ability to gain access to, understand, and use health information in order to promote and maintain good health, per the World Health Organization definition of the construct (2014). In using this measure, as with any other scale or assessment instrument, it is very important to keep in mind what it does measure and what it does not (Eagly \& Chaiken, 1993; Kerlinger, 1986). This measure does not claim to measure the entirety of the individual, system, and population-level factors that contribute to issues surrounding low health literacy (Baker, 2006; Berkman et al., 2011). Rather, the revised POHLS presented in this dissertation offers a self-report of how individuals perceive themselves to be motivated and able to obtain, process, and apply health information from various sources.

In assessing health literacy at the individual level, the revised POHLS addresses one of the four levels forwarded by McCormack and colleagues (2013) for measuring health literacy. As contended by the researchers, health literacy should be assessed at the individual /person, intervention group, patient population/healthcare system, and population levels. Although the revised POHLS does not address all four levels, that is not to say it could not be used to do so using advanced statistical analyses which account for variability at various levels such as hierarchical linear modeling (Tabachnick \& Fidell, 2013). Health literacy is a multidimensional and multifaceted construct (McCormack et al., 2013), and this should be addressed in future studies by either using multiple measures for each of these stages or by using measures such as the revised 
POHLS at multiple levels. The decision of which of these to do will be determined by the needs and intentions of the researcher in the research study at hand.

Further, the revised POHLS should not be confused with or considered synonymous with measures assessing reading comprehension (e.g., REALM; Davis et al., 1993; TOFLA, Parker et al., 1995) or numeracy (NVS; Weiss et al., 2005). Although it does not directly measure these cognitive abilities, it does offer a self-report of an individual's perception of their motivation and ability to perform these tasks; a self-report such as this allows for the integration of one's perceived sense of self in relation to health information, which could be preferable to an objective outcome such as reading grade level, depending on the needs and wants of the researcher. However, if your needs as a researcher include having a precise, objective estimate of reading comprehension and the ability to apply numbers, then using the revised POHLS would not be appropriate. Ideally, researchers could use the more clinically-suited assessments such as the REALM, the TOFLA, or the NVS in a healthcare setting and associate these scores with individual's scores on the revised POHLS. This would allow the researcher to have an objective assessment of reading comprehension and numeracy (i.e., the functional approach to studying health literacy) as well as a more complex assessment of the communicative and interactive skills that determine how an individual functions in the health environment (i.e., a communicative/interactive or critical approach).

As forwarded by McCormack and colleagues (2013) in their recommendations for conducting robust research on health literacy measurement, researchers should use multiple measures of health literacy in a single study, using robust research methods such as multilevel modeling and large samples to forward more large scale efforts in 
measurement design. In line with this recommendation, the revised POHLS should certainly be collected in tandem with other existing measures of health literacy in such a way as to assess its relationship to these measures as well as its strengths and weaknesses in comparison to them. However, if the purpose of the research being conducted is not for measurement analysis, the studies reported in this dissertation suggest that the revised POHLS will suffice as a standalone measure of the construct.

Regardless of whether the revised POHLS is used at a variety of analytical levels or in tandem with other measures, it is very important for future researchers using the measure to be mindful of the ways in which health literacy is socially and culturally situated (Baker, 2006; Paasche-Orlow \& Wolf, 2010). As stated by the Institute of Medicine (IOM, 2004), health literacy is influenced by culture and society, the health system in which an individual exists, and his or her experience in the education system. In its report on health literacy, the IOM (2004) summarized the importance of examining health literacy at the social and cultural level as the following:

Information about health is produced by many sources, including the government and the food and drug industries, and is distributed by the popular media. Commercial and social marketing of health information, products, and services is a multi-billion dollar industry. People are frequently and repeatedly exposed to quick, often contradictory bits of information. This inundation has increased as the Internet has become an increasingly important source of health information. Socioeconomic status, education level, and primary language all affect whether consumers will seek out health information, where they will look, and what type of 
information they prefer, and how they will interpret that information.

Limited health literacy decreases the likelihood that health-related

information will be available to al l (p. 12).

As the IOM report highlighted, it is crucial to consider the social and cultural factors that affect how people obtain and use health information in an ever-changing health environment in order to understand the potential impact of health literacy. Further, the increasing number of competing sources of health information that individuals are exposed to (e.g., the Internet, product marketing, health education, and the national media) intensifies the need to more holistically examine and attempt to improve health literacy (2004).

As mentioned in Chapter I, the study of health literacy has largely been devoid of either theory testing or theory development, part of which may have been explained by the lack of a "gold standard” of health literacy measurement (McCormack et al., 2013, p. 10). As such, another implication of the development and validation of the revised POHLS is its potential to be used in theory-driven research. The association of health literacy and both self- and response- efficacy found in this dissertation, and the role of self-efficacy in major theories of communication (e.g., the Theory of Planned Behavior, Ajzen, 1991; the Transtheoretical Model, Prochaska \& DiClemente, 1983; the Health Beliefs Model, Rosenstock, 1966; the Extended Parallel Processing Model, Witte, 1992) suggests that health literacy could potentially play a role in existing theories designed to explain individuals' attitudes, knowledge, and behaviors related to health. Indeed, Morris and colleagues (2013) found that low health literacy was related to participants’ decreased attitudes toward cancer and health (i.e., more fatalistic attitudes) and decreased 
knowledge of screening tests. Further, individuals with low health literacy were more likely to avoid information regarding diseases they did not have, and were less likely to be up-to-date on colorectal screenings. These findings speak directly to the variables of two of the most widely used theories in the field of Health Communication, the Theory of Reasoned Action (Fishbein \& Ajzen, 1975) and the Theory of Planned Behavior (Ajzen, 1991). Even more important, the way in which health literacy was utilized in this study provides insight into how health literacy could be integrated into theory-driven studies.

The implications of this dissertation also extend to the realm of public health promotion and campaign design. As noted by Mazor and colleagues (2010), many individuals exposed to media messages about cancer prevention on television and the Internet were largely unable to comprehend the information they were exposed to; participants overgeneralized the information they were exposed to (e.g., reporting that preventative behaviors as more protective than the message actually stated), forgot important details such as the recommended age for screening, and confused or misunderstood major concepts such as "early stage” cancer detection (rather than correctly understanding this term as indicating detecting cancer at an early stage of the disease, participants thought this meant the cancer was detected while a person was young). Echoing the concerns of Bernhardt and Cameron (2003), the researchers express their concern that the effectiveness of health messages is being mitigated by the inability of the general public to fully comprehend the information being conveyed. As Mazor and colleagues (2010) assert, further research is needed to determine how to develop more widely accessible health messages. By providing a measure of health literacy that 
assesses not only ability but motivation to access, understand, and apply these health messages, this dissertation could add significantly to these research efforts.

This dissertation also contributed to the growing discussion of how to design interventions to increase health literacy. Although the intervention designed for this purpose of the dissertation was not successful, its implementation and evaluation offered insight for future efforts toward this cause. Notably, the length of the intervention (i.e., 35 minutes) appeared to be too brief to increase individual's motivation and ability to gain access to, understand, and use health information. Therefore, instructing healthcare providers to make efforts to increase their patient's cognitive and social skills in these areas in a less than twenty minute medical interview would not be a worthy endeavor. Rather, health literacy is apparently a facet of the individual that takes time to mold and improve; the optimal length of time needed to do so successfully is yet to be determined. Future research on designing educational interventions to increase health literacy should not only work on content to heighten individuals’ motivation and ability, but also the most efficient means of doing so.

Importantly, the development of the revised POHLS will assist efforts toward designing such interventions. As noted by the IOM (2004), the majority of existing measurements of health literacy assess the construct as reading ability; the results are often discussed in terms of the reading level of participants. These assessments are not only "imprecise at best," they also do not allow for researchers to accurately determine baseline measures of health literacy and monitor changes over time (p. 6-7). As noted by the IOM (2004), there appears to be a desire on the part of adult learners and adult education programs to form partnerships with health communities to increase health 
literacy skills; these desires, however, are impeded by the inadequate training and education of health professionals and staff in these communities. As the revised POHLS is so accessible in its administration and scoring (unlike many existing measures of health literacy), it has the potential to be used for training and education efforts toward this end. As evidenced in Study Four of this dissertation, the scale translated well to being used among adult users of a community center; this holds promise for its use across a wide variety of contexts and samples.

\section{Limitations and Future Directions}

This dissertation is subject to four limitations. The first limitation is that many of the analyses included in the four studies were cross-sectional. The scope and design of the studies reported here were not necessarily interested in how health literacy works as a process. In other words, the cognitive mechanisms that affect health literacy or how health literacy works to affect other health related outcomes was not the focus of the current studies. Rather, health literacy was only examined in terms of its associations with other variables. Although the use of correlational analyses is suitable for determining the validity of a measure (Kerlinger, 1986), these results do not allow for a more comprehensive understanding of how health literacy is situated among various individual, situational, and cultural factors. As discussed by Bailey, McCormack, Rush, and Paasche-Orlow (2013), investigating the mechanisms by which health literacy affects health outcomes is critical to developing a comprehensive understanding of the interrelatedness of health literacy and key socio-demographic characteristics. Using the revised POHLS, future research should work toward building more comprehensive 
models which examine antecedents, outcomes, and potential mediators or moderators of health literacy's impact on individuals' health.

The second limitation is that the revised POHLS does not address every possible indicator of health literacy known in the research literature; the measure offers a valid instrument to assess the communicative or interactive aspect of health literacy, a selfreport of individuals' health literacy, and a general assessment of how motivated and able one perceives him or herself to be in the health environment. As discussed by Baker (2006), an ideal assessment of health literacy would assess individual reading fluency, vocabulary, and health knowledge while simultaneously capturing the difficulty of written materials, the complexity of healthcare provider's speech, and the various written and oral demands individuals face in their particular healthcare environment. An individual's total on each of these indicators would then be indexed for a comprehensive assessment of his or her health literacy. However, as Baker notes, this type of measurement is not practical for most research projects. The revised POHLS offers a much more widely available and accessible measure for use in social science research, that can be used in tandem with other objective measurements of health literacy (e.g., the REALM) if the research question of the project require it. A potential avenue for future research is to examine the association of the revised POHLS with existing measures of the various facets of health literacy (e.g., numeracy, reading ability) to provide further evidence of its validity. As the revised POHLS is a valid measure of an individual's motivation and ability to gain access to, understand, and use health information, it would be fruitful to examine its potentially complex associations with health attitudes, behaviors, and outcomes. 
Upon reviewing the results of Study Two, a third limitation became apparent. The materials used in the educational intervention primarily addressed ability (e.g., how to find good health information, what to ask a healthcare provider during a medical visit, etc.). As increasing individuals' motivation to improve their health is an objective of both federal and private researchers (Berkman et al., 2011), continued efforts should be made to determine effective intervention messages. Relatedly, the university student sample used for the intervention might not have been the most appropriate given their likely low personal relevance for the topic of health literacy. As evidenced in dual process models of persuasion (e.g., the ELM, Petty and Cacioppo, 1986), when personal relevance for a topic is low, individuals are less motivated to actively process information. Perhaps using a community adult sample, and therefore increasing personal relevance, would have led to more success in this study. It should also be noted that the intervention was conducted in a large lecture course; given the research on the effectiveness of tailored messages in persuasive communication (Noar, Harrington, \& Aldrich, 2009; Rimer \& Kreuter, 2006), future attempts to design effective health literacy interventions should focus on individualized messages to increase motivation and ability.

The fourth limitation was the sample obtained for the analyses assessing the predictive validity of the construct (i.e., Study Four; Hypotheses 6 and 7). Although the sample obtained was purposive, as adult residents of West Virginia are highly at risk for obesity and diabetes (CDC, 2011a; HSC, 2012), the lack of significant findings on the analyses related to blood glucose level, Body Mass Index (BMI), and stage of change with behaviors related to physical activity and healthy eating might have occurred for two reasons. First, the sample was relatively small $(N=40)$ for either correlational analysis 
or discriminatory function analyses (Field, 2009). Using this small sample size, it is possible that we did not have the statistical power to detect a significant relationship among these variables. Second, no participant in the sample was hypo- or hyperglycemic, and the majority of participants fell into the normal BMI range. This lack of variability, which is not representative of the population from which the sample was drawn (see Manchin et al., 2009), makes it more difficult to determine if a relationship exists between health literacy and these health-related risk factors. Future research needs to obtain a sample with greater variability in order to assess the predictive validity of the revised POHLS in relation to these physiological and psychological indicators of health.

Related to the above, this study used an individual's BMI as an absolute indicator of health. This is problematic, as an individuals’ BMI is a relative indicator of health and is also influenced by a number of factors. As discussed by the CDC (2011b), BMI is a useful screening tool to identify possible weight problems, but it is not a diagnostic tool. In order to officially diagnose a person as overweight or obese, a healthcare provider would need to make various other assessments such as skin fold thickness measurements, evaluations of diet and physical activity, family history, and other health screenings (2011b). Further, the BMI is not a direct assessment of body fat percentage; highly trained athletes may have a high BMI (i.e., fall in the obese category) because of increased muscularity. However, as the CDC (2011b) notes, although some people in the “overweight” range (i.e., a BMI of 25.0-29.9) might not be overweight due to the measure's inability to account for muscle mass, individuals who have a BMI of over 30 and are therefore categorized as “obese” definitely have higher than normal levels of body fat. Future research which assesses BMI as a physiological health outcome should 
also look to longitudinal designs to obtain a relative indicator of an individual's BMI. Experimental designs which use a pretest-posttest design would be able to assess the relative change in an individual's BMI over time and how this associates with changes in health literacy. Overall, the BMI allows for an easy and inexpensive means of comparing one's health to that of the general population, which makes it a viable option for future researchers to use as an indicator of physiological health.

\section{Conclusion}

The field of health literacy spans a number of disciplines, including but not limited to medicine, communication studies, pharmacy, and nursing (Bailey et al., 2013). Although much progress has been made across these disciplines in understanding health literacy, scholars have struggles to find a mutually agreed upon measurement of the construct (Bailey et al., 2013; McCormack et al., 2013), particularly one which addresses the complexity of health literacy as a communicative and dynamic phenomenon (Nutbeam, 2000). This dissertation contributes to the literature by offering a valid and reliable measure of health literacy which is conceptually consistent with a leading definition of the construct.

The 26-item revised POHLS is a two factor measure which assesses individuals' motivation and ability to gain access to, understand, and use health information in order to promote and maintain good health. This is consistent with the definition of Health Literacy provided by the World Health Organization (2014). The measure performed admirably across four samples and demonstrated strong content and construct validity. Although the criterion-related validity was not able to be established for the measure within this dissertation, this is a rich avenue for future research using the measure. 
Further, the longitudinal pretest-posttest design conducted to assess the ability to influence health literacy is certainly a strength of this dissertation, as health literacy scholars have recently begun to call for an increasing focus on developing successful interventions to increase health literacy as opposed to the multitude of studies which examine the association of health literacy to various health outcomes (Bailey et al., 2013). Importantly, there is some evidence within this dissertation that health literacy is a skill set that educational efforts can effectively increase, although further research is needed to determine the most effective means of doing so. Overall, this dissertation makes important contributions to the study of health literacy by developing and validating a measure of health literacy that can be used in social science research. It is the intention of this dissertation to facilitate and inspire future examinations on health literacy to achieve a more nuanced understanding of the construct as well as how to increase it to help individuals achieve improved health outcomes. 


\section{NOTES}

1. Although the final 26 item version of the Revised Perceived Oral Health Literacy Scale was used in all analyses for Study Two and Study Three, the original 74 item pool measure was administered to both of these samples. This is due to the concurrent data collections for Studies One, Two, and Three (i.e., the data used in the confirmatory factor analysis that was used to determine a final factor structure for the scale was a part of Study Two data collection). Study Three data was collected before the analysis for Studies One and Two were complete.

2. The Flesch-Kincaid Grade Level and Flesch Reading Ease indices (Kincaid, Rishburne, Rogers, \& Chissom, 1975) have been established as valid and reliable means of assessing the readability of text. The index assesses readability on the basis of the average number of syllables per word and the average number of words per sentence. The grade level is based on school levels in the United States and ranges from 0-12. A grade level of 5.0 means that a fifth grade student can understand the text. Most documents aim for a grade level of 7.0 to 8.0. The formula for the Flesch-Kincaid Grade Level score is as follows:

$$
(.39 \times \mathrm{ASL})+(11.8 \times \mathrm{ASW})-15.59
$$

where:

ASL = Average sentence length (the number of words divided by the number of sentences)

ASW = Average number of syllables per word (the number of syllables divided by the number of words). 
The Flesch Reading Ease Test rates text on a 100-point scale. The higher the score, the easier it is to understand the document. For most files, a score between 60 and 70 is preferred. The formula for the Flesch Reading Ease test is as follows:

$$
206.835-(1.015 \times \text { ASL })-(84.6 \times \text { ASW })
$$

where:
ASL = Average sentence length (the number of words divided by the number of sentences)
ASW = Average number of syllables per word (the number of syllables divided by the number of words).

These indices are automated in Microsoft Word. 


\section{References}

Agre, P., Steiglitz, E., \& Milstein, G. (2006). The case for development of a new test of health literacy. Oncology Nursing Forum, 33, 283-289. doi:10.1188/06.ONF.283289

Allen, M., \& Bourhis, J. (1996). The relationship of communication apprehension to communication behavior: A meta-analysis. Communication Quarterly, 44, 214266. doi:10.1080/01463379609370011

American Medical Association, Ad Hoc Committee on Health Literacy for the Council on Scientific Affairs. (1999). Health literacy: Report of the Council of Scientific Affairs. Journal of the American Medical Association, 281, 552-557.

An, S., \& Muturi, N. (2011). Subjective health literacy and older adults' assessment of direct-to-consumer prescription drug ads. Journal of Health Communication, 16, 242-255. doi:10.1080/10810730.2011.604387

Arozullah, A. M., Yarnold, P. R., Bennett, C. L., Soltysik, R. C., Wolf, M. S., Ferreira, R. M., ...Davis, T. (2007). Development and validation of a short-form, rapid estimate of adult literacy in medicine. Medical Care, 45, 1026-1033. doi:10.1097/MLR.0b013e3180616c1b

Austin, E. W., \& Johnson, K. K. (1997). Immediate and delayed effects of media literacy training on third graders' decision making for alcohol. Health Communication, 9, 323-349. doi:10.1207/s15327027hc0904_3

Ayres, J., Colby-Rotell, Wadleigh, P. M., \& Hopf, T. (1996). Measuring patients’ communication apprehension about interacting with physicians: Instrument development. Communication Research Reports, 13, 86-93. 
doi:10.1080/08824099609362074

Ajzen, I. (1991). The theory of planned behavior. Organizational Behavior and Human Decision Processes, 50, 179-211.

Bailey, S. C., McCormack, L. A., Rush, S. R., \& Paasche-Orlow, M. K. (2013). The progress and promise of health literacy research. Journal of Health Communication, 18, 5-8. doi:10.1080/10810730.2013.830042

Baker, D. W. (2006). The meaning and measure of health literacy. Journal of General Internal Medicine, 21, 878-883. doi:10.1111/j.1525-1497.2006.00540.x.

Baker, D. W., Gazmararian, J. A., Williams, M. V., Scott, T., Parker, R. M., Ren, J., \& Peel, J. (2002). Functional health literacy and the risk of hospital admission among Medicare managed care enrollees. American Journal of Public Health, 92, 1278-1283. doi:10.2105/AJPH.92.8.1278

Baker, D. W., Parker, R. M., Williams, M. V., \& Clark, W. S. (1998). Health literacy and the risk of hospital admission. Journal of General Internal Medicine, 13, 791-798. doi:10.1046/j.1525-1497.1998.00242.x

Baker, D. W., Parker, R. M., Williams, M. V., Clark, W. S., \& Nurss, J. (1997). The relationship of patient reading ability to self-reported health and use of health services. American Journal of Public Health, 87, 1027-1030. doi:10.2105/AJPH.87.6.1027

Baker, D. W., Williams, M. V., Parker, R. M., Gazmararian, J. A., \& Nurss, J. (1999). Development of a brief test to measure functional health literacy. Patient Education and Counseling, 33, 33-42. doi:10.1016/S0738-3991(98)00116-5

Bandura, A. (1977). Social learning theory. Englewood Cliffs, NJ: Prentice-Hall. 
Barrett, P. (1986). Factor comparison: An examination of three methods. Personality and Individual Differences, 7, 327-340.

Bass, P. F. III., Wilson, J. F., \& Griffith, C. H. (2003). A shortened measure for literacy screening. Journal of General Internal Medicine, 18, 1036-1038. doi:10.1111/j.1525-1497.2003.10651.x

Battersby, C., Hartley, K., Fletcher, A. E., Markowe, H. J., Brown, R. G., Styles, W., ...\& Fraser, S. (1993). Cognitive function in hypertension: A community based study. Journal of Human Hypertension, 7, 117-123.

Beatty, M. J., Pascual-Ferra, P., \& Levine, T. (2013, November). Two studies examining the error theory underlying the undimensional treatment of the verbal aggressiveness scale. Paper presented at the annual meeting of the National Communication Association, Washington, D.C.

Behrens, S. J. (1994). A conceptual analysis and historical overview of information literacy. College and Research Libraries, 55, 309-322.

Benjamin, R. (2010). Health literacy improvement as a national priority. Journal of Health Communication, 15, 1-3. doi:10.1080/10810730.2010.499992

Bentler, P. M. (1990). Comparative fit indexes in structural models. Psychological Bulletin, 107, 238-246. doi:10.1037/0033-2909.107.2.238

Bentler, P. M. \& Bonnet, D. G. (1980). Significance tests and goodness of fit in the analysis of covariance structures. Psychological Bulletin, 88, 588-606.

Berkman, N. D., Davis, T. C., \& McCormack, L. (2010). Health literacy: What is it?. Journal of Health Communication, 15, 9-19. doi:10.1080/10810730.2010.499985

Berkman, N. D., Sheridan, S. L., Donahue, K. E., Halpern, D. J., \& Crotty, K. (2011). 
Low health literacy and health outcomes: An updated systematic review. Annals of Internal Medicine, 155, 97-107. doi:10.7326/0003-4819-155-2-20110719000005

Bernhardt, J. M., \& Cameron, K. A. (2003). Accessing, understanding, and applying health communication messages: The challenge of health literacy. In T. L. Thompson, A. Dorsey, K. I. Miller, and R. Parrott (Eds.), Handbook of health communication (pp. 583-605). New York: Routledge.

Bickmore, T. W., Pfeifer, L. M., Byron, D., Forsythe, S., Henault, L. E., Jack, B. W., ... \& Paasche-Orlow, M. K. (2010). Usability of conversational agents by patients with inadequate health literacy: Evidence from two clinical trials. Journal of Health Communication, 15, 197-210. doi:10.1080/10810730.2010.499991

Bodenheimer, T., Lorig, K., Holman, H., \& Grumbach, K. (2002). Patient selfmanagement of chronic disease in primary care. JAMA: The Journal of the American Medical Association, 288, 2469-2475. doi: 10.1001/jama.288.19.2469

Bollen, K.A. (1989). A new incremental fit index for general structural equation models. Sociological Methods and Research, 17, 303-316.

Booth-Butterfield, M. (2003). Integrating health communication pedagogy, social science, and health interventions. Communication Quarterly, 51,332-350. doi:10.1080/01463370309370160

Booth-Butterfield, M. (2008). Communication apprehension and social anxiety. In W. Donsbach (Ed.), The International Encyclopedia of Communication. doi:10.1111/b.9781405131995.2008.x 
Booth-Butterfield, M., \& Booth-Butterfield, S. (1986). The effect of evaluation, task structure, trait-CA, and reticence on state-CA and behavioral disruption in dyadic settings. Communication Monographs, 53, 144-159.

doi:10.1080/03637758609376133

Booth-Butterfield, S., \& Gould, M. (1986). The communication anxiety inventory: Validation of state- and context-communication apprehension. Communication Quarterly, 34, 194 -205. doi:10.1080/01463378609369633

Brann, M., Himes, K. L., Dillow, M. R., \& Weber, K. (2010). Dialectical tensions in stroke survivor relationships. Health Communication, 25, 323-332. doi:10.1080/10410231003773342

Browne, M.W. \& Cudeck, R. (1993). Alternative ways of assessing model fit. In K. A. Bollen \& J. S. Long (Eds.), Testing structural equation models (pp.136-162). Newbury Park, CA: Sage.

Bute, J. J., Donovan-Kicken, E., \& Martins, N. (2007). Effects of communicationdebilitating illnesses and injuries on close relationships: A relational maintenance perspective. Health Communication, 21, 235-246.

doi:10.1080/10410230701307675

Bute, J. J., \& Vik, T. A. (2010). Privacy management as unfinished business: Shifting boundaries in the context of infertility. Communication Studies, 61, 1-20. doi:10.1080/10510970903405997

Byrne, B. M. (2001). Structural equation modeling with AMOS: Basic concepts, applications, and programming. Mahwah, NJ: Erlbaum.

Cafiero, M. (2013). Nurse practitioners’ knowledge, experience, and intention to use 
health literacy strategies in clinical practice. Journal of Health Communication, 18, 70-81. doi:10.1080/10810730.2013.825665

Callaghan, R. C., Hathaway, A., Cunningham, J. A., Vettese, L. C., Wyatt, S., \& Taylor, L. (2005). Does stage-of-change predict dropout in a culturally diverse sample of adolescents admitted to inpatient substance-abuse treatment? A test of the Transtheoretical Model. Addictive Behaviors, 30, 1834-1847. doi:10.1016/j.addbeh.2005.07.015

Cameron, K. A., Wolf, M. S., \& Baker, D. W. (2011). Integrating health literacy in health communication. In T. L. Thompson, R. Parrott, \& J. F. Nussbaum (Eds.), The Routledge handbook of health communication (2 ${ }^{\text {nd }}$ ed.), (pp. 306-319), New York: Routledge.

Campbell, D. T., \& Fiske, D. W. (1959). Convergent and discriminant validation by the multitrait-multimethod matrix. Psychological Bulletin, 56, 81-105. doi:10.1037/h0046016

Capella, J. N. (2006). Integrating message effects and behavior change theories:

Organizing comments and unanswered questions. Journal of Communication, 56, S265-S279. doi:10.1111/j.1460-2466.2006.00293.x

Cegala, D. J. (2006). Emerging trends and future directions in patient communication skills training. Health Communication, 20, 123-129. doi:10.1207/s15327027hc2002_3

Cegala, D. J., \& Broz, S. L. (2003). Provider and patient skills training. In T. L. Thompson, A. Dorsey, K. I. Miller, and R. Parrott (Eds.), Handbook of health communication (pp. 583-605). New York: Routledge. 
Center for Disease Control and Prevention (CDC, 2011a). National diabetes fact sheet: National estimates and general information on diabetes and prediabetes in the United States. Retrieved from: http://www.cdc.gov/DIABETES/pubs/pdf/ndfs_2011.pdf

Center for Disease Control and Prevention (CDC, 2011b). About BMI for adults. Center for Disease Control and Prevention. Retrieved from: http://www.cdc.gov/healthyweight/assessing/bmi/adult_bmi/index.html Center for Disease Control and Prevention (CDC, 2013a). Overweight and obesity: Adult obesity facts. Retrieved from: http://www.cdc.gov/obesity/data/adult.html Center for Disease Control and Prevention (CDC; 2013b). Body mass index. Center for Disease Control and Prevention. Retrieved from: http://www.cdc.gov/healthyweight/assessing/bmi/

Cohen, R. J., Swerdlik, M. E., \& Sturman, E. D. (2010). Psychological testing and assessment: An introduction to tests and measurement ( $8^{\text {th }}$ ed.). New York: McGraw-Hill.

Cortés, D. E., Drainoni, M-L., Henault, L. E., \& Paasche-Orlow, M. K. (2010). How to achieve informed consent for research from Spanish-speaking individuals with low health literacy: A qualitative report. Journal of Health Communication, 15, 172-182. doi:10.1080/10810730.2010.499990

Cronbach, L. J., \& Meehl, P. E. (1955). Construct validity in psychological tests. Psychological Bulletin, 52, 281-302. doi:10.1037/h0040957

Davis, T. C., Arnold, C., Berkel, H. J., Nandy, I., Jackson, R. H., \& Glass, J. (1996). Knowledge and attitude on screening mammography among low-literate, 
low-income women. Cancer, 78, 1912-1920. doi:10.1002/(SICI)10970142(19961101)78:9<1912::AID-CNCR11>3.0.CO;2-0

Davis, T. C., Dolan, N. C., Ferreira, M. R., Tomori, C., Green, K. W., Sipler, A. M., \& Bennett, C. L. (2001). The role of inadequate health literacy skills in colorectal cancer screening. Cancer Investigation, 19, 193-200. doi:10.1081/CNV100000154

Davis, T. C., Long, S. W., Jackson, R. H., Mayeaux, E. J., George, R. B., Murphy, P. W., \& Crouch, M. A.(1993). Rapid estimate of literacy in medicine: A shortened screening instrument. Family Medicine, 25, 391-395.

Davis, T. C., Michielutte, R., Askov, E. N., Williams, M. V., \& Weiss, B. D. (1998). Practical assessment of adult literacy in health care. Health Education Behavior, 25, 613-624. doi:10.1177/109019802500508

Davis, T. C., Wolf, M. S., Bass, P. F., Thompson, J. A., Tilson, H. H., Neuberger, M., \& Parker, R. M. (2006). Literacy and misunderstanding prescription drug labels. Annals of Internal Medicine, 145, 887-894. doi:10.7326/0003-4819-145-12200612190-00144

Dembrowski, P. (1968). Finite Geometrics. Berlin, Germany: Springer Verlag.

DeVellis, R. F. (2012). Scale development: Theory and applications ( $3^{\text {rd }}$ ed.). Los Angeles: Sage.

DeWalt, D. A., Berkman, N. D., Sheridan, S., Lohr, K. N., \& Pignone M. P. (2004). Literacy and health outcomes: A systematic review of the literature. Journal of General Internal Medicine, 19, 1228-1239. doi:10.1111/j.15251497.2004.40153.x 
Dickens, C., Lambert, B. L., Cromwell, T., \& Piano, M. R. (2013). Nurse overestimation of patients’ health literacy. Journal of Health Communication, 18, 62-69. doi:10.1080/10810730.2013.825670

Doak, C. C., Doak, L. G., Friedell, G. H., \& Meade, C. D. (1998). Improving comprehension for cancer patients with low literacy skills: strategies for clinicians. CA: A Cancer Journal for Clinicians, 48, 151-162. doi:10.3322/canjclin.48.3.151

Donovan-Kicken, E., Mackert, M., Guinn, T. D., Tollison, A. C., Breckinridge, B., \& Pont, S. J. (2012). Health literacy, self-efficacy, and patients' assessment of medical disclosure and consent documentation. Health Communication, 27, 581590. doi:10.1080/10410236.2011.618434

Duncan, C., Miller, D. M., Borskey, E. J., Fomby, B., Dawson, P., \& Davis, L. (2002). Barriers to safer sex practices among African American college students. Journal of the National Medical Association, 94,944-951. doi:

Eagly, A. H., \& Chaiken, S. (1993) The psychology of attitudes. New York: Harcourt Brace Jovanovich College Publishers.

Evans, B. J., Stanley, R. O., \& Burrows, G. D. (1992). Communication skills training and patients' satisfaction. Health Communication, 4, 155-170. doi:10.1207/s15327027hc0402_5

Field, A. (2009). Discovering statistics using SPSS ( $3^{\text {rd }}$ ed.). Washington, DC: Sage. Fischer, R. C., Vauclair, M., Fontaine, J. R. J., \& Schwartz, S. H. (2010). Are individuallevel and country-level value structures different? Testing Hofstede’s legacy with 
the Schwartz value survey. Journal of Cross-Cultural Psychology, 41, 135-151. doi:10.1177/0022022109354377

Fishbein, M., \& Ajzen, I. (1975). Belief, attitude, intention and behavior: An introduction to theory and research. Reading, MA: Addison-Wesley.

Fleming, M. F., Balousek, S. L., Grossberg, P. M., Mundt, M. P., Brown, D., Wiegel, J. R., ...Saewyc, E. M. (2010). Brief physician advice for heavy drinking college students: A randomized controlled trial in college health clinics. Journal of Studies on Alcohol and Drugs, 1, 23-31. doi:

Floyd, K., \& Morman, M. T. (1998). The measurement of affectionate communication. Communication Quarterly, 46, 144-162. doi:10.1080/01463379809370092

Frates, J., Bohrer, G. G., \& Thomas, D. (2006). Promoting organ donation to Hispanics: the role of the media and medicine. Journal of Health Communication, 11, 683698. doi:10.1080/10810730600934542

Gardiner, P., Mitchell, S., Filippelli, A. C., Sadikova, E., White, L. F., Paasche-Orlow, M. K., \& Jack, B. W. (2013). Health literacy and complementary and alternative medicine use among underserved inpatients in a safety net hospital. Journal of Health Communication, 18, 290-297. doi:10.1080/10810730.2013.830663

Gazmararian, J., Baker, D., Parker, R., \& Blazer, D. G. (2000). A multivariate analysis of factors associated with depression. Archives of Internal Medicine, 160, 33073314. doi:10.1001/archinte.160.21.3307

Gazmararian, J. A., Kripalani, S., Miller, M. J., Echt, K. V., Ren, J., \& Rask, K. (2006). Factors associated with medication refill adherence in cardiovascular-related 
diseases. Journal of General Internal Medicine, 21, 1215-1221.

doi:10.1111/j.1525-1497.2006.00591.x

Gazmararian, J. A., Parker, R. M., \& Baker, D. W. (1999). Reading skills and family planning knowledge and practices in a low-income managed-care population. Obstetrics \& Gynecology, 93, 239-244. doi:10.1016/S0029-7844(99)00237-9

Gerber, B. S., Brodsky, I. G., Lawless, K. A., Smolin, L. I., Arozullah, A. M., Smith, E. V., ... Eiser, A. R. (2005). Implementation and evaluation of a low-literacy diabetes education computer multimedia application. Diabetes Care, 28, 15741580. doi:10.2337/diacare.28.7.1574

Golbeck, A. L., Ahlers-Schmidt, C. R., Paschal, A. M., \& Dismuke, S. E. (2005). A definition and operational framework for health numeracy. American Journal of Preventive Medicine, 29, 375-376. doi:10.1016/j.amepre.2005.06.012

Goodboy, A. K., Martin, M. M., \& Bolkan, S. (2009). The development and validation of the student communication satisfaction scale. Communication Education, 58, 372396. doi:10.1080/03634520902755441

Gorely, T., \& Gordon, S. (1995). An examination of the transtheoretical model and exercise behavior in older adults. Journal of Sport \& Exercise Psychology, 17, 312-324.

Gossett, D. R., Nayak, S., Bhatt, S., \& Bailey, S. C. (2013). What do healthy women know about the consequences of delayed childbearing? Journal of Health Communication, 18, 118-128. doi:10.1080/10810730.2013.825677

Graham, J., Bennett, I. M., Holmes, W. C., \& Gross, R. (2006). Medication beliefs as mediators of the health literacy-antiretroviral adherence relationship in HIV- 
infected individuals. AIDS and Behavior, 11, 385-392. doi:10.1007/s10461-0069164-9

Haas, S. M. (2002). Social support as relationship maintenance in gay male couples coping with HIV or AIDS. Journal of Social and Personal Relationships, 19, 87111. doi:10.1177/025407502191005

Hale, J. L., \& Trumbetta, S. L. (1996). Women’s self-efficacy and sexually transmitted disease preventative behaviors. Research in Nursing and Health, 19, 101-110. doi:10.1002/(SICI)1098-240X(199604)19:2<101::AID-NUR2>3.0.CO;2-Q

Harrington, N. G., Norling, G. R., Witte, F. M., Taylor, J., \& Andrews, J. E. (2007). The effects of communication skills training on pediatricians' and parents' communication during “sick child” visits. Health Communication, 21, 105-114. doi:10.1080/10410230701306974

Hatcher, L. (1994). A step by step approach to using the SAS system for factor analysis and structural equation modeling. Cary, NC: SAS Institute Incorporated.

Hawthorne, G. (1996). Preteenage drug use in Australia: The key predictors and schoolbased drug education. Journal of Adolescent Health, 20, 384-395.

Health Statistics Center. (HSC; 2012). Diabetes and health equity in West Virginia: A Review. West Virginia Department of Health and Human Resources. Retrieved from:http://www.wvdhhr.org/bph/hsc/pubs/briefs/028/Brief28_20121220_Health _Eq_S tat.pdf

Hobbs, R. (1998). The seven great debates in the media literacy movement. Journal of Communication, 48, 16-32. doi:10.1111/j.1460-2466.1998.tb02734.x 
Holman, H., \& Lorig, K. (2004). Patient self-management: a key to effectiveness and efficiency in care of chronic disease. Public Health Reports, 119, 239-243. doi:10.1016/j.phr.2004.04.002

Hopf, T., \& Ayres, J. (1992). Coping with public speaking anxiety: An examination of various combinations of systematic desensitization, skills training, and visualization. Journal of Applied Communication Research, 20, 183-198. doi:10.1080/00909889209365328

Hu, L., \& Bentler, P. M. (1999). Cutoff criteria for fit indexes in covariance structure analysis: conventional criteria versus new alternatives. Structural Equation Modeling, 6, 1-55. doi:10.1080/10705519909540118

Huang, S. J., Hung, W. C., Chang, M., \& Chang, J. (2009). The effect of an internetbased, stage-matched message intervention on young Taiwanese women's physical activity. Journal of Health Communication, 14, 210-227. doi:10.1080/10810730902805788

Hwang, Y. (2012). Social diffusion of campaign effects: Campaign-generated interpersonal communication as a mediator of antitobacco campaign effects. Communication Research, 39, 120-141. doi:10.1177/0093650210389029 Institute of Medicine (IOM). (2004). Health literacy: A prescription to end confusion (L. Nielsen-Bohlman, A. Panzer, \& D. A. Kindig, Eds.). Washington, DC: National Academies Press.

Johnson, S. E., Baur, C., \& Meissner, H. I. (2011). Back to basics: Why basic research is needed to create effective health literacy interventions. Journal of Health Communication, 16, 22-29. doi:10.1080/10810730.2011.604707 
Johnson, S. S., Driskell, M. M., Johnson, J. L., Dyment, S. J., Prochaska, J. O., Prochaska, J. M., \& Bourne, L. (2006). Transtheoretical model intervention for adherence to lipid-lowering drugs. Disease Management, 9, 102-114. doi:10.1089/dis.2006.9.102

Kalichman, S. C., Pope, H., White, D., Cherry, C., Amaral, C. M., Swetzes, C., ... \& Kalichman, M. O. (2008). The association between health literacy and HIV treatment adherence: Further evidence from objectively measured medication adherence. Journal of the International Association of Physicians in AIDS Care, 7, 317-323. doi:10.1177/1545109708328130

Kalichman, S., C., Ramachandran, B., \& Catz, S. (1999). Adherence to combination antiretroviral therapies in HIV patients of low health literacy. Journal of General Internal Medicine, 14, 267-273. doi:10.1046/j.1525-1497.1999.00334.x

Kalichman, S. C., \& Rompa, D. (2000). Functional health literacy is associated with health status and health-related knowledge in people living with HIV-AIDS. Journal of Acquired Immune Deficiency Syndromes, 24, 337-344. doi:10.1097/00126334-200012010-00007

Kelly, L. (1989). Implementing a skills training program for reticent communicators. Communication Education, 38, 85-101. doi:10.1080/03634528909378743

Kerlinger, F. N. (1986). Foundations of behavioral research ( $3^{\text {rd }}$ ed.). New York: Holt, Rinehart, and Winston, Inc.

Kincaid, J. P., Fishburne, Jr. R. P., Rogers, R. L., \& Chissom, B. S. (1975). Derivation of new readability formulas (Automated Reliability Index, Fog Count, and Flesch 
Reading Ease Formula) for Navy Enlisted Personnel. Memphis: Naval Technical Training Command Research Branch Report.

Kline, K. N. (2007). Cultural sensitivity and health promotion: Assessing breast cancer education pamphlets designed for African American women. Health Communication, 21, 85-96. doi:10.1080/10410230701283454

Kline, R. B. (2011). Principles and practice of structural equation modeling. New York,: Guilford Press.

Koch-Weser, S., Rudd, R. E., \& DeJong, W. (2010). Quantifying word use to study health literacy in doctor-patient communication. Journal of Health Communication, 15, 590-602. doi:10.1080/10810730.2010.499592

Kreps, G. L. (1989). Setting the agenda for health communication research and development: Scholarship that can make a difference. Health Communication, 1, 11-15. doi:10.1207/s15327027hc0101_2

Kreps, G. L. (2001). The evolution and advancement of health communication inquiry. In W. B. Gudykunst (Ed.), Communication Yearbook, 24, (pp.232-254). Newbury Park, CA: Sage

Kreps, G. L., Bonaguro, E. W., \& Query Jr, J. L. (1998). The history and development of the field of health communication. In L. D. Jackson \& B. K. Duffy (Eds.), Health communication research: Guide to developments and directions (pp. 1-15), Westport, CT: Greenwood Press.

Kutner, M., Greenberg, E., \& Baer, J. (2005). A first look at the literacy of America's adults in the $21^{\text {st }}$ century. Washington, DC: National Center for Education Statistics, U.S. Department of Education. 
Kutner, M., Greenberg, E., Jin, Y., \& Paulsen, C. (2006). The Health Literacy of America's Adults: Results from the 2003 National Assessment of Adult Literacy (NCES Report No. 2006-483). Washington, D.C.: Government Printing Office.

LaBelle, S., \& Weber, K. (2013, April). Development and validation of the Perceived Oral Health Literacy Scale. Paper presented at the annual meeting of the Eastern Communication Association, Pittsburgh, PA.

Langevin, M. (2009). The peer attitudes toward children who stutter scale: Reliability, known groups validity, and negativity of elementary school-age children's attitudes. Journal of Fluency Disorders, 34, 72-86.

doi:10.1016/j.jfludis.2009.05.001

Lanning, B. A., \& Doyle, E. I. (2010). Health literacy: developing a practical framework for effective health communication. American Medical Writers Association Journal, 25, 155-161.

Lee, S. Y. D., Bender, D. E., Ruiz, R. E., \& Cho, Y. I. (2006). Development of an Easy-to-Use Spanish Health Literacy Test. Health Services Research, 41, 13921412. doi:10.1111/j.1475-6773.2006.00532.x

Leung, A. Y. M., Cheung, M. K. T., Lou, V. W., Q., Chan, F. H. W., Ho, C. K. Y., Do, T. L., ...Chi, I. (2013). Development and validation of the Chinese health literacy scale for chronic care. Journal of Health Communication, 18, 205-222. doi:10.1080/10810730.2013.829138

Lincoln, A. K., Arford, T., Prener, C., Garverich, S., \& Koenen, K. C. (2013). The need for trauma-sensitive language use in literacy and health literacy screening 
instruments. Journal of Health Communication, 18, 15-19.

doi:10.1080/10810730.2013.825676

Livingstone, S. (2004). Media literacy and the challenge of new information and communication technologies. The Communication Review, 7, 3-14. doi:10.1080/10714420490280152

MacCallum, R. C., Browne, M. W., \& Sugawara, H. M. (1996). Power analysis and determination of sample size for covariance structure modeling. Psychological Methods, 1, 130-149. doi:10.1037/1082-989X.1.2.130

Mackert, M., Champlin, S. E., Pasch, K. E., \& Weiss, B. D. (2013). Understanding health literacy measurement through eye tracking. Journal of Health Communication, 18, 185-196. doi:10.1080/10810730.2013.825666

Manchin, J., Hardy, P. A., Curtis, C., Bazzle, N. M., \& Slemp, C. (2009). The burden of diabetes in West Virginia 2009. Charleston, WV: West Virginia Department of Health and Human Services.

Manganello, J. A., \& Clayman, M. L. (2011). The association of understanding of medical statistics with health information seeking and health provider interaction in a national sample of young adults. Journal of Health Communication, 16, 163176. doi:10.1080/10810730.2011.604704

Marcus, B. H., \& Simkin, L. R. (1994). The transtheoretical model: applications to exercise behavior. Medicine \& Science in Sports \& Exercise, 26, 1400-1404. doi:10.1249/00005768-199411000-00016

Marks, R., \& Allegrante, J. P. (2005). A review and synthesis of research evidence for self-efficacy-enhancing interventions for reducing chronic disability: implications 
for health education practice (part II). Health Promotion Practice, 6, 148-156. doi:10.1177/1524839904266792

Martin, L. T., Schonlau, M., Haas, A., Derose, K. P., Rosenfeld, L., Buka, S. L., \& Rudd, R. (2011). Patient activation and advocacy: which literacy skills matter most? Journal of Health Communication, 16, 177-190.

doi:10.1080/10810730.2011.604705

Mazor, K. M., Calvi, J., Cowan, R., Costanza, M. E., Han, P. K., Greene, S. M., ... \& Williams, A. (2010). Media messages about cancer: what do people understand? Journal of Health Communication, 15, 126-145. doi:10.1080/10810730.2010.499983

McArdle, F. B. (2000). Public policy and public health: Making the connection. In Health literacy: Advancing public policy (pp. 13-17). New York: Pfizer.

McCombs, M., \& Shaw, D. (1972). The agenda-setting function of mass media. Public Opinion Quarterly, 36, 176-187. doi:10.1086/267990

McCormack, L., Bann, C., Squiers, L., Berkman, N. D., Squire, C., Schillinger, D., ... \& Hibbard, J. (2010). Measuring health literacy: a pilot study of a new skills-based instrument. Journal of Health Communication, 15, 51-71.

doi:10.1080/10810730.2011.605823

McCormack, L., Haun, J., Sorensen, K., \& Valerio, M. (2013). Recommendations for advancing health literacy measurement. Journal of Health Communication, 18, 914. doi:10.1080/10810730.2013. 829892 
McCroskey, J. C. (1977). Oral communication apprehension: A summary of recent theory and research. Human Communication Research, 4, 78-96. doi:10.1111/j.1468-2958.1977.tb00599.x

McCroskey, J. C. (2008). Communication apprehension: What have we learned in the last four decades. Human Communication, 12, 179-187.

McCroskey, J. C., \& Beatty, M. J. (1986). Oral communication apprehension. In W. H. Jones, J. M. Cheek, \& S. R. Briggs (Eds.), Shyness: Perspectives on research and treatment (pp. 279-293). New York: Plenum Press.

McCroskey, J.C., \& Beatty, M.J. (1998). Communication apprehension. J. C. McCroskey, J. A. Daly, M. M. Martin, \& M. J. Beatty. (Eds.), Communication and personality (pp. 215-231). Cresskill, NJ: Hampton Press.

Morris, N. S., Field, T. S., Wagner, J. L., Cutrona, S. L., \& Roblin, D. W. (2013). The association between health literacy and cancer-related attitudes, behaviors, and knowledge. Journal of Health Communication, 18, 223-241. doi:10.1080/10810730.2013.825667

Morrow, D., Clark, D., Tu, W., Wu, J., Weiner, M., Steinley, D., \& Murray, M. D. (2006). Correlates of health literacy in patients with chronic heart failure. The Gerontologist, 46, 669-676. doi:10.1093/geront/46.5.669

Muir, K. W., Santiago-Turla, C., Stinnett, S. S., Herndon, L. W., Allingham, R. R., Challa, P., \& Lee, P. P. (2006). Health literacy and adherence to glaucoma therapy. American Journal of Ophthalmology, 142, 223-226. doi:10.1016/j.ajo.2006.03.018

Murphy, P. W., Davis, T. C., Long, S. W., Jackson, R. H., \& Decker, B. C. (1993). Rapid 
estimate of adult literacy in medicine (REALM): A quick reading test for patients. Journal of Reading, 37, 124-130.

Naar-King, S., Wright, K., Parsons, J. T., Frey, M., Templin, T., \& Ondersma, S. (2006). Transtheoretical model and condom use in HIV-positive youths. Health Psychology, 25, 648-652. doi:10.1037/0278-6133.25.5.648

National Diabetes Education Program. (2014). Risk factors and warning signs for diabetes. U. S. Department of Health and Human Services. Retrieved from: http://ndep.nih.gov/am-i-at-risk/DiabetesRiskFactors.aspx

National Literacy Act of 1991. United States Congress, Committee on Education and Labor.

Ngoh, L. N. (2009). Health literacy: A barrier to pharmacist-patient communication and medication adherence. Journal of American Pharmacists Association, 49, e132e149. doi:10.1331/JAPhA.2009.07075

Noar, S. M. (2006). A 10-year retrospective of research in health mass media campaigns: Where do we go from here? Journal of Health Communication, 11, 21-42. doi:10.1080/10810730500461059

Noar, S. M., Harrington, N. G., \& Aldrich, R. S. (2009). The role of message tailoring in the development of persuasive health communication messages. Communication Yearbook, 33, 73-133.

Nutbeam, D. (2000). Health literacy as a public health goal: A challenge for contemporary health education and communication strategies into the $21^{\text {st }}$ century. Health Promotion International, 15, 259-267. doi:10.1093/heapro/15.3.259 
Nutbeam, D. (2008). The evolving concept of health literacy. Social Science and Medicine, 67, 2072-2078. doi:10.1016/j.socscimed.2008.09.050

Ogden, C. L., Carroll M. D., Kit, B. K., \& Flegal, K. M. (2012). Prevalence of obesity in the United States, 2009-2010. NCHS data brief, no 82. Hyattsville, MD: National Center for Health Statistics.

Olson, R. M., Blank, D., Cardinal, E., Hopf, G., \& Chalmers, R. K. (1996). Understanding medication-related needs of low-literacy patients. Journal of the American Pharmaceutical Association (Washington, DC), 424-429.

Ong, L. M. L., de Haes, J. C. J. M., Hoos, A. M., \& Lammes, F. B. (1995). Doctorpatient communication: A review of the literature. Social Science Medicine, 40, 903-918. doi:10.1016/0277-9536(94)00230-Q

Osborn, C. Y., Cavanaugh, K., Wallston, K. A., Kripalani, S., Elasy, T. A., Rothman, R. L., \& White, R. O. (2011). Health literacy explains racial disparities in diabetes medication adherence. Journal of Health Communication, 16, 268-278. doi:10.1080/10810730.2011.604388

Osborn, C. Y., Cavanaugh, K., Wallston, K. A., \& Rothman, R. L. (2010). Self-efficacy links health literacy and numeracy to glycemic control. Journal of Health Communication, 15, 146-158. doi:10.1080/10810730.2010.499980

Paasche-Orlow, M. K., Parker, R. M., Gazmararian, J. A., Nielsen-Bohlman, L. T., \& Rudd, R. R. (2005). The prevalence of limited health literacy. Journal of General Internal Medicine, 20, 175-184. doi:10.1111/j.1525-1497.2005.40245.x 
Paasche-Orlow, M. K., \& Wolf, M. S. (2010). Promoting health literacy research to reduce health disparities. Journal of Health Communication, 15, 34-41. doi:10.1080/10810730.2010.499994

Parikh, N. S., Parker, R. M., Nurss, J. R., Baker, D. W., \& Williams, M. V. (1996). Shame and health literacy: the unspoken connection. Patient Education and Counseling, 27, 33-39. doi:10.1016/0738-3991(95)00787-3

Parker, R. M., Baker, D. W., Williams, M. V., \& Nurss, J. R. (1995). The test of functional health literacy in adults: A new instrument for measuring patients' literacy skills. Journal of General Internal Medicine, 10, 537-541. doi:10.1007/BF02640361

Parker, R. M., Williams, M. V., Baker, D. W., \& Nurss, J. R. (1996). Literacy and contraception: exploring the link. Obstetrics \& Gynecology, 88, 72S-77S. doi:10.1016/0029-7844(96)00250-5

Parker, R., \& Ratzan, S. C. (2010). Health literacy: A second decade of distinction for Americans. Journal of Health Communication, 15, 20-33. doi:10.1080/10810730.2010.501094

Parker, R. M., Ratzan, S. C., \& Lurie, N. (2003). Health literacy: a policy challenge for advancing high-quality health care. Health Affairs, 22, 147-153. doi:10.1377/hlthaff.22.4.147

Petty, R. E., \& Cacioppo, J. T. (1986). Communication and Persuasion: Central and Peripheral Routes to Attitude Change. New York: Springer-Verlag.

Pignone, M., DeWalt, D. A., Sheridan, S., Berkman, N., \& Lohr, K. N. (2005). Interventions to improve health outcomes for patients with low literacy: A 
systematic review. Journal of General Internal Medicine, 20, 185-192.

doi:10.111/j.1525-1497.2005.40208.x

Plax, T. G., Kearney, P., McCroskey, J. C., \& Richmond, V. P. (1986). Power in the classroom VI: Verbal control strategies, nonverbal immediacy and affective learning. Communication Education, 35, 43-55. doi:10.1080/03634528609388318

Pleasant, A., McKinney, J., \& Rikard, R. V. (2011). Health literacy measurement: A proposed research agenda. Journal of Health Communication, 16, 11-21. doi:10.1080/10810730.2011.604392

Portney, L. G., \& Watkins, M. P. (2000). Foundations of clinical research: Applications to practice. Upper Saddle River, NJ: Prentice Hall Health.

Potter, W. J. (2012). Media literacy. Thousand Oaks, CA: Sage.

Press, V. G., Shapiro, M. I., Mayo, A. M., Meltzer, D. O., \& Arora, V. M. (2013). More than meets the eye: Relationship between low health literacy and poor vision in hospitalized patients. Journal of Health Communication, 18, 197-204. doi:10.1080/1080730.2013.830346

Prochaska, J. O., \& DiClemente, C. C. (1982). Transtheoretical therapy: Toward a more integrative model of change. Psychotherapy: Theory, Research \& Practice, 19, 276.

Prochaska, J. O., \& DiClemente, C. C. (1983). Stages and processes of self-change of smoking: Toward and integrative model of change. Journal of Consulting and Clinical Psychology, 51, 390-395. doi:10.1037/0022-006X.51.3.390

Prochaska, J. O., Redding, C. A., Harlow, L. L., Rossi, J. S., \& Velicer, W. F. (1994). The transtheoretical model of change and HIV prevention: A review. Health 
Education \& Behavior, 21, 471-486. doi:10.1177/109019819402100410

Rancer, A. S., Whitecap, V. G., Kosberg, R. L., \& Avtgis, T. A. (1997). Testing the efficacy of a communication training program to increase argumentativeness and argumentative behavior in adolescents. Communication Education, 46, 273-286. doi:10.1080/03634529709379101

Ratzan, S. C. (2011). Health literacy: Building upon a strong foundation. Journal of Health Communication, 16, 3-4. doi:10.1080/10810730.2011.606071

Ratzan, S. C. (2013). Preface: Note from the editor. Journal of Health Communication, 18, 1-2. doi:10.1080/10810730.2013.837365

Redding, C. A., Morokoff, P. J., Rossi, J. S., \& Meier, K. S. (2009). A TTM-tailored condom use intervention for at-risk women and men. In T. Edgar, S. M. Noar, \& V. S. Freimuth (Eds.), Communication perspectives on HIV/AIDS for the 21st century (pp. 423-428). New York: Lawrence Erlbaum.

Rice, R. E., Stewart, L. P., \& Hujber, M. (2000) Extending the domain of instructional effectiveness assessment in student evaluations of communication courses. Communication Education, 49, 253-266. doi:10.1080/03634520009379214

Richmond, V. P., Smith, Jr. R. S., Heisel, A. M., \& McCroskey, J. C. (1998). The impact of communication apprehension and fear of talking with a physician on perceived medical outcomes. Communication Research Reports, 15, 344-353. doi:10.1080/08824099809362133

Rimal, R. N., \& Real, K. (2003). Perceived risk and efficacy beliefs as motivators of change. Human Communication Research, 29, 370-400. doi:10.1002/bdm.528 
Rimer, B. K., \& Kreuter, M. W. (2006). Advancing tailored health communication: A persuasion and message effects perspective. Journal of Communication, 56, S184S201. doi: 10.1111/j.1460-2466.2006.00289.x

Roberts, S. T., \& Kennedy, B .L. (2006). Why are young college women not using condoms? Their perceived risk, drug use, and developmental vulnerability may provide important clues to sexual risk. Archives of Psychiatric Nursing, 20, 32-40. doi: 10.1016/j.apnu.2005.08.008

Robinson, J. P., Shaver, P. R., \& Wrightsman, L. S. (1991). Measures of personality and social psychological attitudes: Volume 1 in measures of social psychological attitudes series. London, UK: Elsevier.

Robinson-Smith, G., \& Pizzi, E. R. (2003). Maximizing stroke recovery using patient self-care self-efficacy. Rehabilitation Nursing, 28, 48-51. doi:10.1002/j.20487940.2003.tb02028.x

Rogers, R. W. (1975). A protection motivation theory of fear appeals and attitude change. The Journal of Psychology, 91, 93-114. doi: 10.1080/00223980.1975.9915803

Rogers, E. M. (2004). A prospective and retrospective look at the diffusion model. Journal of Health Communication, 9, 13-19. doi:10.1080/10810730490271449

Rosenstock, I. M. (1966). Why people use health services. The Milbank Memorial Fund Quarterly, 44, 94-127. doi:10.2307/3348967

Roter, D. (2000). The enduring and evolving nature of the patient-physician relationship. Patient Education and Counseling, 39, 5-15. doi:10.1016/S0738-3991(99)000865

Rubin, D. L., Parmer, J., Freimuth, V., Kaley, T., \& Okundaye, M. (2011). Associations 
between older adults' spoken interactive health literacy and selected health care and health communication outcomes. Journal of Health Communication, 16, 191204. doi:10.1080/10810730.2011.604380

Rudd, R., Kirsch, I., \& Yamamoto, K. (2004). Literacy and health in America. Princeton, NJ: Educational Testing Service.

Samora, J., Saunders, L., \& Larson, R. F. (1961). Medical vocabulary knowledge among hospital patients. Journal of Health and Human Behavior, 83-92. doi:10.2307/2948804

Sanders, L. M., Shaw, J. S., Guez, G., Baur, C., \& Rudd, R. (2009). Health literacy and child health promotion: Implications for research, clinical care, and public policy. Pediatrics, 124, S306-S314. doi:10.1542/peds.2009-1162G.

Sarkin, J. A., Johnson, S. S., Prochaska, J. O., \& Prochaska, J. M. (2001). Applying the transtheoretical model to regular moderate exercise in an overweight population: validation of a stages of change measure. Preventive Medicine, 33, 462-469. doi:10.1006/pmed.2001.0916

Schillinger, D., Grumbach, K., Piette, J., Wang, F., Osmond, D., Daher, C., ... \& Bindman, A. B. (2002). Association of health literacy with diabetes outcomes. Journal of the American Medical Association, 288, 475-482. doi:10.1001/jama.288.4.475

Sentell, T., Braun, K. L., Davis, J., \& Davis, T. (2013). Colorectal cancer screening: Low health literacy and limited English proficiency among Asians and Whites in California. Journal of Health Communication, 18, 242-255.

doi:10.1080/10810730.2013.825669 
Sequeira, S. S., Eggermont, L. H. P., Silliman, R. A., Bickmore, T. W., Henault, L. E., Winter, M. R., ... Paasche-Orlow, M. K. (2013). Limited health literacy and decline in executive function in older adults. Journal of Health Communication, 18, 143-157. doi:10.1080/1080730.2013.825673

Shone, L. P., King, J. P., Doane, C., Wilson, K. M., \& Wolf, M. S. (2011). Misunderstanding and potential unintended misuse of acetaminophen among adolescents and young adults. Journal of Health Communication, 16, 256-267. doi:10.1080/10810730.2011.604384

Schonlau, M., Martin, L., Haas, A., Derose, K. P., \& Rudd, R. (2011). Patients' literacy skills: More than just reading ability. Journal of Health Communication, 16, 1046-1054. doi:10.1080/10810730.2011.571345

Schwartz, L. M., Woloshin, S., \& Welch, H. G. (2005). Can patients interpret health information? An assessment of the medical data interpretation test. Medical Decision Making, 25, 290-300. doi:10.1177/0272989X05276860

Schwartz, L. M., Woloshin, S., Black, W. C., \& Welch, H. G. (1997). The role of numeracy in understanding the benefit of screening mammography. Annals of Internal Medicine, 127, 966-972. doi:10.7326/0003-4819-127-11-19971201000003

Sharf, B. F. (1999). The present and future of health communication scholarship: Overlooked opportunities. Health Communication, 11, 195-199. doi:10.1207/s15327027hc1102_5

Singleton, R. A., Straits, B. C., \& Straits, M. M. (1993). Approaches to social research. New York: Oxford University Press. 
Slater, M. D. (1999). Integrating application of media effects, persuasion, and behavior change theories to communication campaigns: A stages-of-change framework. Health Communication, 11, 335-354.doi;10.1207/S15327027HC1104_2

Smith, M. S., Wallston, K. A., \& Smith, C. A. (1995). The development and validation of the perceived health competence scale. Health Education Practice, 10, 51-64. doi:10.1093/her/10.1.51

Spencer, L., Adams, T. B., Malone, S., Roy, L., \& Yost, E. (2006). Applying the transtheoretical model to exercise: a systematic and comprehensive review of the literature. Health Promotion Practice, 7, 428-443. doi:10.1177/1524839905278900

Spencer, L., Pagell, F., \& Adams, T. (2005). Applying the transtheoretical model to cancer screening behavior. American Journal of Health Behavior, 29, 36-56. doi:10.5993/AJHB.29.1.4

Spencer, L., Pagell, F., Hallion, M. E., \& Adams, T. B. (2002). Applying the transtheoretical model to tobacco cessation and prevention: a review of literature. American Journal of Health Promotion, 17, 7-71. doi:10.4278/0890-1171-17.1.7

Steiger, J. H. \& Lind, J. C. (1980, May 30). Statistically-based tests for the number of common factors. Paper presented at the Annual Spring Meeting of the Psychometric Society, Iowa City.

Strecher, V. J., DeVellis, B. M., Becker, M. H., \& Rosenstock, I. M. (1986). The role of self-efficacy in achieving health behavior change. Health Education \& Behavior, 13, 73- 92. doi:10.1177/109019818601300108

Sudore, R. L., Schillinger, D., Knight, S. J., \& Fried, T. R. (2010). Uncertainty about 
advance care planning treatment preferences among diverse older adults. Journal of Health Communication, 15, 159-171. doi:10.1080/10810730.2010.499982

Sullivan, L. M., Dukes, K. A., Harris, L., Dittus, R. S., Greenfield, S., \& Kaplan, S. H. (1995). A comparison of various methods of collecting self-reported health outcomes data among low-income and minority patients. Medical Care, 33, AS183-AS194.

Tabachnick, B. G., \& Fidell, L. S. (2013). Using multivariate statistics (6 ${ }^{\text {th }}$ ed.). Boston: Pearson.

Ten Berge, J. M. F. (1986). Some relationships between descriptive comparisons of components from different studies. Multivariate Behavioral Research, 21, 29-40.

Thompson, T. L., Parrott, R., \& Nussbaum, J. F. (Eds.). (2011). The Routledge handbook of health communication ( $2^{\text {nd }}$ ed.). New York: Routledge.

Torres, R. Y., \& Marks, R. (2009). Relationships among health literacy, knowledge about hormone therapy, self-efficacy, and decision-making among postmenopausal health. Journal of Health Communication, 14, 43-55.

doi:10.1080/10810730802592247

Trauth, J. M., Ling, B. S., Weissfeld, J. L., Schoen, R. E., \& Hayran, M. (2003). Using the transtheoretical model to stage screening behavior for colorectal cancer. Health Education \& Behavior, 30, 322-336. doi:10.1177/1090198103030003007

Tuckson, R. (2004). Understanding health disparities. Columbus, OH: Health Policy Institute of Ohio. Retrieved from: http://a5e8c023c8899218225edfa4b02e4d9734e01a28.gripelements.com/pdf/publ ications/healthdisparities.pdf 
U. S. Census Bureau (2012). State and Country Quick Facts: West Virginia. U.S. Census Bureau. Retrieved from: http://quickfacts.census.gov/qfd/states/54000.html U. S. Census Bureau (2014). Annual estimates of the resident population: April 1, 2010 to July 1, 2013. U. S. Census Bureau, Population Division. Retrieved from: http://factfinder2.census.gov/faces/tableservices/jsf/pages/productview.xhtml?src $=\mathrm{bkmk}$

U. S. Department of Health and Human Services. (1998) Clinical guidelines on the identification, evaluation, and treatment of overweight and obesity in adults: The evidence report. National Institute of Health. Retrieved from: http://www.nhlbi.nih.gov/guidelines/obesity/ob_gdlns.pdf

U.S. Department of Health and Human Services, Office of Disease Prevention and Health Promotion (2000). Healthy people 2010. Retrieved from healthypeople.gov.

U.S. Department of Health and Human Services, Office of Disease Prevention and Health Promotion (2013a). Health Communication and Health Information Technology: National Snapshot. Retrieved from http://healthypeople.gov/2020/topicsobjectives2020/nationalsnapshot.aspx?topicI $d=18$

U.S. Department of Health and Human Services, Office of Disease Prevention and Health Promotion (2013b). Health communication and health information technology: Topic and objectives. Retrieved from http://healthypeople.gov/2020/topicsobjectives2020/overview.aspx?topicid=18

U.S. Department of Health and Human Services, Office of Disease Prevention and Health Promotion (2013c). Diabetes: Overview. Retrieved from 
http://www.healthypeople.gov/2020/topicsobjectives2020/overview.aspx?topicId $=29$

U.S. Department of Health and Human Services, Office of Disease Prevention and Health Promotion (2013d). Nutrition and weight status: Overview. Retrieved from http://www.healthypeople.gov/2020/topicsobjectives2020/overview.aspx?topicid $=8$

Ussher, M., Ibrahim, S., Reid, F., Shaw, A., \& Rowlands, G. (2010). Psychosocial correlates of health literacy among older patients with coronary heart disease. Journal of Health Communication, 15, 788-804. doi:

$10.1080 / 10810730.2010 .514030$

Valente, T. W. (1996). Mass-media-generated interpersonal communication as sources of information about family planning. Journal of Health Communication, 1, 247266. doi:10.1080/108107396128040

Vallis, M., Ruggiero, L., Greene, G., Jones, H., Zinman, B., Rossi, S., ... \& Prochaska, J. O. (2003). Stages of change for healthy eating in diabetes: Relation to demographic, eating- related, health care utilization, and psychosocial factors. Diabetes Care, 26, 1468-1474. doi:10.2337/diacare.26.5.1468

van der Heide, I., Wang, J., Droomers, M., Spreeuwenberg, P., Rademakers, J., \& Uiters, E. (2013). The relationship between health, education, and health literacy: Results from the Dutch adult literacy and life skills survey. Journal of Health Communication, 18, 172-184. doi:10.1080/10810730.2013.825668

Velasquez, M. M., Carbonari, J. P., \& Diclemente, C. C. (1999). Psychiatric severity and behavior change in alcoholism: the relation of the transtheoretical model variables 
to psychiatric distress in dually diagnosed patients. Addictive Behaviors, 24, 481496. doi:10.1016/S0306-4603(98)00103-8

Vernon, J. A., Trujillo, A., Rosenbaum, S., \& DeBuono, B. (2007). Low health literacy: Implications for National Policy (2000). Retrieved August 12, 2013 from http://gwumc.edu/sphhs/departments/healthpolicy/dhp_publications/pub_uploads/ dhpPublication_3AC9A1C2-5056-9D20-3D4BC6786DD46B1B.pdf

Waldrop, D., Lightsey Jr, O. R., Ethington, C. A., Woemmel, C. A., \& Coke, A. L. (2001). Self-efficacy, optimism, health competence, and recovery from orthopedic surgery. Journal of Counseling Psychology, 48, 233-238. doi:10.1037/00220167.48.2.233

Wallace, L. S., Rogers, E. S., Roskos, S. E., Holiday, D. B., \& Weiss, B. D. (2006). Brief report: screening items to identify patients with limited health literacy skills. Journal of General Internal Medicine, 21, 874-877. doi:10.1111/j.15251497.2006.00532.x

Webber, S., \& Johnston, B. (2000). Conceptions of information literacy: new perspectives and implications. Journal of Information Science, 26, 381-397. doi:10.1177/016555150002600602

Weber, K., \& Martin, M. M. (2012). Designing and evaluating the campus organ donor project. Communication Quarterly, 60, 504-519. doi:10.1080/01463373.2012.704575

Weber, K., Martin, M. M., \& Cayanus, J. L. (2005). Student interest: A two-study reexamination of the concept. Communication Quarterly, 53, 71-86. doi:10.1080/01463370500055996 
Weber, K., Martin, M., \& Patterson, B. (2001). Teacher behavior, student interest and affective learning: Putting theory to practice. Journal of Applied Communication Research, 29, 71-90. doi:10.1080/00909880128101

Weber, K. D., \& Patterson, B. R. (1996). Construction and validation of a communication based emotional support scale. Communication Research Reports, 13, 68-76. doi:10.1080/08824099609362072

Weber, K., \& Patterson, B. R. (2000). Student interest, empowerment, and motivation. Communication Research Reports, 17, 22-29. doi:10.1080/08824090009388747

Weber, K. M., \& Solomon, D. H. (2008). Locating relationship and communication issues among stressors associated with breast cancer. Health Communication, 23, 548-559. doi:10.1080/10410230802465233

Weiss, B. D., Blanchard, J. S., McGee, D. L., Hart, G., Warren, B., Burgoon, M., \& Smith, K. J. (1994). Illiteracy among Medicaid recipients and its relationship to health care costs. Journal of Health Care for the Poor and Underserved, 5, 99111. doi:10.1353/hpu.2010.0272

Weiss, B. D., Mays, M. Z., Martz, W., Castro, K. M., DeWalt, D. A., Pignone, M. P., ... \& Hale, F. A. (2005). Quick assessment of literacy in primary care: The newest vital sign. Annals of Family Medicine, 3, 514-522. doi:10.1370/afm.405

Weiss, B. D., \& Palmer, R. (2004). Relationship between health care costs and very low literacy skills in a medically needy and indigent Medicaid population. Journal of the American Board of Family Practice, 17, 44-47. doi:10.3122/jabfm.17.1.44

Wheeless, V. E. (1984). Communication apprehension and trust as predictors of willingness to discuss gynecological health topics. Communication Research 
Reports,1, 117-121.

Williams, M. V., Parker, R. M., Baker, D. W., Parikh, N. S., Pitkin, K., Coates, W. C., \& Nurss, J. R. (1995). Inadequate functional health literacy among patients at two public hospitals. Journal of the American Medical Association, 274, 1677-1682.

Williams, M. V., Baker, D. W., Honig, E. G., Lee, T. M., \& Nowlan, A. (1998). Inadequate literacy is a barrier to asthma knowledge and self-care. Chest, 114, 1008-1015. doi:10.1378/chest.114.4.1008

Williams, M. V., Baker, D. W., Parker, R. M., \& Nurss, J. R. (1998). Relationship of functional health literacy to patients' knowledge of their chronic disease. A study of patients with hypertension and diabetes. Archives of Internal Medicine, 158, 166-172. doi:10.1001/archinte.158.2.166.

Williams, M. V., Davis, T., Parker, R., \& Weiss, B. D. (2002). The role of health literacy in patient-physician communication. Communicating with Special Populations, 34, 383-389.

Wilson, E. A., H., Wolf, M. S., Curtis, L. M., Clayman, M. L., Cameron, K. A., Cameron, K. A., ...\& Makoul, G. (2010). Literacy, cognitive ability, and the retention of health-related information about colorectal cancer screening. Journal of Health Communication, 15, 116-125. doi:10.1080/10810730.2010.499984

Witte, K. (1992). Putting the fear back into fear appeals: The extended parallel process model. Communication Monographs, 59, 329-349. doi:10.1080/03637759209376276

Wolf, M. S., Davis, T. C., Tilson, H. H., Bass, III P. F., \& Parker, R. M. (2006). Misunderstanding of prescription drug warning labels among patients with low 
literacy. American Journal of Health-System Pharmacy, 63, 1048-1055.

World Health Organization Commission on the Social Determinants of Health (WHO;2007). Achieving health equity: From root causes to fair outcomes.

Geneva: World Health Organization. Retrieved from http://www.who.int/socialdeterminants/resources/interimstatement/en/index.html.

World Health Organization (WHO; 2010). Global recommendations on physical activity for health. World Health Organization. Retrieved from: http://whqlibdoc.who.int/publications/2010/9789241599979_eng.pdf?ua=1

World Health Organization (WHO; 2013a). Track 2: Health Literacy and Health Behavior. World Health Organization: $7^{\text {th }}$ Global Conference on Health Promotion. Retrieved from: http://www.who.int/healthpromotion/conferences/7gchp/track2/en/\# World Health Organization (WHO; 2013b). Obesity and overweight: Fact Sheet. Available from http://www.who.int/mediacentre/factsheets/fs311/en/index.html World Health Organization (WHO; 2014). Track 2: Health Literacy and Health Behavior. World Health Organization: $7^{\text {th }}$ Global Conference on Health Promotion. Retrieved from: http://www.who.int/healthpromotion/conferences/7gchp/track2/en/\#

Wynia, M. K., \& Osborn, C. Y. (2010). Health literacy and communication quality in health care organizations. Journal of Health Communication, 15, 102-115. doi:10.1080/10810730.2010.499981 
Young, M., \& Klingle, R. S. (1996). Silent partners in medical care: a cross-cultural study of patient participation. Health Communication, 8, 29-53. doi:10.1207/s15327027hc0801_2

Yost, K. J., Webster, K., Baker, D. W., Jacobs, E. A., Anderson, A., \& Hahn, E. A. (2010). Acceptability of the talking touchscreen for health literacy assessment. Journal of Health Communication, 15, 80-92. doi:10.1080/10810730.2010.500713

Zettl, H. (1998). Contextual media aesthetics as the basis for media literacy. The Journal of Communication, 48, 81-95. doi:10.1111/j.1460-2466.1998.tb02739.x 


\title{
APPENDICES
}

\author{
Appendix A
}

The Rapid Estimate of Adult Literacy in Medicine (REALM)

Table 1

Rapid Estimate of Adult Literacy in Medicine (REALM)

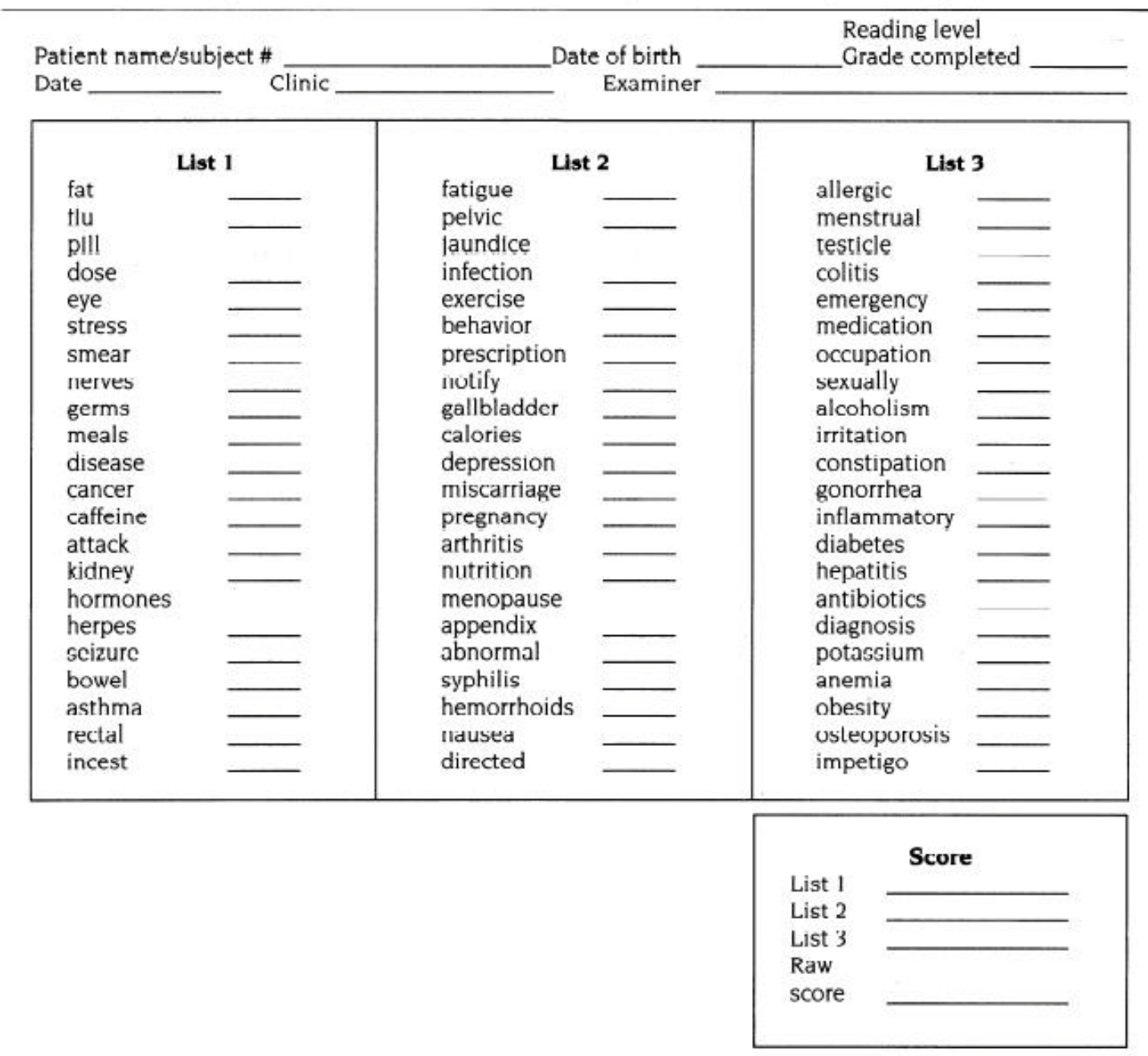

Note: While the set of words used in REALM is copyrighted, ite authors are placing the teet in the public domain to encourage ite use by physicians and reading specialists. Plastic laminated copies of the word list and examiner coples with instructions are available from Peggy Murphy. Department of Internal Medicine, LSUMC-S, PO Box 33932. Shreveport, LA 71130-3932, USA. Telephone: (318) 674-5813. 
Appendix B

The Rapid Estimate of Adult Literacy in Medicine Short Form (REALM-SF)

\section{Rapid Estimate of Adult Literacy in Medicine-Short Form (REALM-SF)}

Suggested Introduction: "We are studying medical word reading in order to improve communication between healthcare providers and patients. Here is a list of medical words that may be difficult to read."

Interviewer: Show the participant the Word List.

Then say, "Starting at the top of the list, please read each word aloud to me. If you don't recognize a word, you can say 'pass' and move on to the next word. Your results will be kept strictly confidential and will not be included in your official medical records."

Interviewer: If the participant takes more than 5 seconds on a word, say "pass" and point to the next word. Hold this scoring sheet so that it is not visible to the participant.

\begin{tabular}{|c|c|c|c|}
\hline Fat & Not scored & & \\
\hline Flu & Not scored & & \\
\hline 1. Behavior & $1 \square$ Correct & ${ }_{2} \square$ Mispronounced & ${ }_{3} \square$ Not attempted \\
\hline 2. Exercise & $\square$ Correct & ${ }_{2} \square$ Mispronounced & $3 \square$ Not attempted \\
\hline 3. Menopause & $\square$ Correct & ${ }_{2} \square$ Mispronounced & $3 \square$ Not attempted \\
\hline 4. Rectal & $\square$ Correct & ${ }_{2} \square$ Mispronounced & ${ }_{3} \square$ Not attempted \\
\hline 5. Antibiotics & $\square$ Correct & ${ }_{2} \square$ Mispronounced & ${ }_{3} \square$ Not attempted \\
\hline 6. Anemia & $\square$ Correct & ${ }_{2} \square$ Mispronounced & ${ }_{3} \square$ Not attempted \\
\hline 7. Jaundice &,$\square$ Correct & ${ }_{2} \square$ Mispronounced & $3 \square$ Not attempted \\
\hline
\end{tabular}

REALM-SF Scoring

\begin{tabular}{|cc|}
\hline $\begin{array}{c}\text { Total Correct } \\
(0-7)\end{array}$ & Grade Level \\
\hline 0 & $\leq 3^{\text {rt }}$ grade \\
\hline $1-3$ & $4^{\text {th }}-6^{\text {th }}$ grade \\
\hline $4-6$ & $7^{\text {th }}-8^{\text {th }}$ grade \\
\hline 7 & $\geq 9^{\text {th }}$ grade \\
\hline
\end{tabular}


Appendix C

The Short Test of Functional Literacy in Adults (STOFLA)

Numeracy Item 1: (Label on prescription bottle)

Take one tablet by mouth every 6 hours as needed.

ORAL QUESTION: If you take your first tablet at 7:00 a.m., when should you take the next one?

CORRECT ANSWER: 1:00 p.m.

Numeracy Item 2: (Prompt Card)

Normal blood sugar is 60-150. Your blood sugar is 160 .

ORAL QUESTION: If this was your score, would your blood sugar be normal today? CORRECT ANSWER: No.

Numeracy Item 3: (Prompt Card)

CLINIC APPOINTMENT

CLINIC: Diabetic

LOCATION: 3rd floor

DAY: Thurs. DATE: April $2^{\text {nd }}$

Issued by:
HOUR: 10:20 a.m.

p.m.

\section{YOU MUST BRING YOUR PLASTIC CARD WITH YOU}

ORAL QUESTION: When is your next appointment?

CORRECT ANSWER: April $2^{\text {nd }}$ or Thursday, April $2^{\text {nd }}$.

Numeracy Item 4: (Label on Prescription Bottle)

Take medication on empty stomach one hour before or two to three hours after a meal unless otherwise directed by your doctor.

ORAL QUESTION: If you eat lunch at 12:00 noon, and you want to take this medicine before lunch, what time should you take it?

CORRECT ANSWER: 11:00 or before 11:00 
Reading Comprehension Passage:

1. Your doctor has sent you to have a X-ray.

a. Stomach

b. Diabetes

c. Stitches

d. Germs

2. You must have an stomach when you come for
a. Asthma
a. is
b. Empty
b. am
c. Incest
c. if
d. Anemia
d. it

3. The $x$-ray will from 1 to 3 to do.
a. Take
a. beds
b. View
b. brains
c. Talk
c. hours
d. Look
d. diets

\section{THE DAY BEFORE THE X-RAY}

4. For supper have only a snack of fruit, and jelly, with coffee or tea.

a. Little
a. toes
b. throat
c. toast
d. thigh

b. Broth

c. Attack

d. Nausea 


\section{Appendix D}

The Newest Vital Sign (NVS)

This information is on the back of a container of a pint of ice cream.

\begin{tabular}{|c|c|c|}
\hline $\begin{array}{l}\text { Nutrition Facts } \\
\text { Serving Size } \\
\text { Servings per container }\end{array}$ & & $\begin{array}{r}1 / 2 \text { cup } \\
4\end{array}$ \\
\hline \multicolumn{3}{|l|}{ Amount per serving } \\
\hline & & $\%$ DV \\
\hline Total Fat $13 \mathrm{~g}$ & & $20 \%$ \\
\hline Sat Fat $9 g$ & & $40 \%$ \\
\hline Cholesterol $28 \mathrm{mg}$ & & $12 \%$ \\
\hline Sodium $55 \mathrm{mg}$ & & $2 \%$ \\
\hline Total Carbohydrate $30 \mathrm{~g}$ & & $12 \%$ \\
\hline \multicolumn{3}{|l|}{ Dietary Fiber $2 \mathrm{~g}$} \\
\hline Sugars $23 g$ & & \\
\hline Protein $4 \mathrm{~g}$ & & $8 \%$ \\
\hline
\end{tabular}

*Percentage Daily Values (DV) are based on a

2,000 calorie diet. Your daily values may

be higher or lower depending on your

calorie needs.

Ingredients: Cream, Skim Milk, Liquid

Sugar, Water, Egg Yolks, Brown Sugar, Milkfat, Peanut Oil, Sugar, Butter, Salt, Carrageenan, Vanilla Extract. 
Please fill in the appropriate answer in the spaces provided below.

1. If you eat the entire container, how many calories will you eat?

2. If you are allowed to eat 60 grams of carbohydrates as a snack, how much ice cream could you have?

3. Your doctor advises you to reduce the amount of saturated fat in your diet. You usually have $42 \mathrm{~g}$ of saturated fat each day, which includes one serving of ice cream. If you stop eating ice cream, how many grams of saturated fat would you be consuming each day?

4. If you usually eat 2,500 calories in a day, what percentage of your daily value of calories will you be eating if you eat one serving?

Pretend that you are allergic to the following substances: penicillin, peanuts, latex gloves, and bee stings.

5. Is it safe for you to eat this ice cream? Please write "yes" or "no.”

6. If not, why not? 


\section{Appendix E}

\section{Original Perceived Oral Health Literacy Scale}

Instructions: With your healthcare provider in mind, please use the scale below to indicate your agreement to the following set of statements. Write your answer in the space provided. There is neither a right nor a wrong answer.

In this study, the term healthcare provider is used to represent the person you usually see for your health needs. This might be a general health practitioner, a specialist, or a nurse. As you answer the questions on this survey, keep in mind the healthcare provider you see most often for your health needs.

\begin{tabular}{|c|c|c|c|c|}
\hline $\begin{array}{c}\text { Strongly } \\
\text { Disagree }\end{array}$ & Disagree & $\begin{array}{c}\text { Neither } \\
\text { Disagree nor } \\
\text { Agree }\end{array}$ & Agree & Strongly Agree \\
\hline 1 & 2 & 3 & 4 & 5 \\
\hline
\end{tabular}

1. I feel confident that I am able to apply the medical directives given to me by my healthcare provider when I leave his or her office.

2. I am confident in my ability to ask questions of my healthcare provider in conversation with him or her.

3. When I leave an interaction with my healthcare provider, I am confident that I recall the key points of his or her message.

4. I directly ask questions of my healthcare provider.

5. When I have a question for my healthcare provider, I make sure I ask it.

6. I often leave interactions with my healthcare provider feeling confused.

7. When I leave interactions with my healthcare provider, I feel like I have said everything that I wanted to say.

8. If I do not understand a term that my healthcare provider has used, I do not hesitate to ask him or her to clarify.

9. I often feel my time with my healthcare provider was not long enough. (Recoded)

10. I leave interactions with my healthcare provider feeling very informed about my health. 


\section{Appendix F}

\section{Pilot Test Questionnaire}

The following series of statements reflect your experiences with the information you receive every day regarding your health. This might include, but is not limited to, articles that you read in the newspaper or a magazine regarding health-related choices, articles on the internet, news stories on the internet or on television, and even the conversations that you have with friends, family, and healthcare providers on how to improve and maintain good health. Healthcare providers may include your general physician, a health specialist that you see on a regular basis, a nurse, or even individuals you see in urgent care situations.

\begin{tabular}{|c|c|c|c|c|}
\hline $\begin{array}{c}\text { Strongly } \\
\text { Disagree }\end{array}$ & Disagree & $\begin{array}{c}\text { Neither Disagree } \\
\text { nor Agree }\end{array}$ & Agree & Strongly Agree \\
\hline 1 & 2 & 3 & 4 & 5 \\
\hline
\end{tabular}

Please take a moment to reflect on the health information that you receive from these sources. Then, indicate in the space provided how much you agree with each of the following statements using the scale below.

1. I feel confident that I am able to apply the medical instructions given to me by a healthcare provider when I leave his or her office.

2. I adapt the health information I see and heat to my own life.

3. I confident in my ability to ask questions of a healthcare provider in conversation with him or her.

4. I listen to suggestions from people I trust on how to be healthy.

5. I am able to understand the directions a healthcare provider gives me.

6. I apply health information to my own life and health choices when possible.

7. When I leave an interaction with a healthcare provider, I am confident that I recall the key points of his or her message.

8. I use the advice my doctor gives me on how to improve my health.

9. I am motivated to understand the directions given to me by a healthcare provider.

10. I often use "tips" I hear on how to live a healthy life.

11. I directly ask questions of healthcare providers. 
12. I am able to use the health information I receive from multiple sources.

13. When I have a question for a healthcare provider, I make sure I ask it.

14. I have a hard time applying suggestions I hear on how to live a healthy lifestyle.

15. I worry that healthcare providers will think my questions are "dumb."

16. I am not able to figure out how to apply the instructions a healthcare provider gives me once I am home.

17. When a healthcare provider is talking to me, I am able to understand most of what he or she is saying.

18. When I hear information on how to improve my health or live a healthy life, I am able to apply it to my own situation.

19. When I leave a healthcare provider's office, I remember exactly what he or she told me to do.

20. I am able to apply the suggestions I receive from others on how to improve my health.

21. I am confident in my ability to listen to a healthcare provider when he or she is talking.

22. I do not care to see if the health suggestions others give me work to improve my health.

23. I fully listen to a healthcare provider when he or she is talking.

24. I am not interested in using the health information that I see every day.

25. When I ask a healthcare provider a question, he or she fully listens to me.

26. I am motivated to follow the advice my healthcare provider gives me about my health.

27. I often leave interactions with a healthcare provider feeling confused.

28. I am interested in applying messages I receive from others on how to improve my health.

29. When I leave interactions with a healthcare provider, I feel like I have said everything that I wanted to say.

30. I want to use the health information I see every day to live a healthy life.

31. I understand the terms a healthcare provider uses in interactions with me.

32. I am motivated to apply information that I receive to improve my health.

33. If I do not understand a term that a healthcare provider has used, I do not hesitate to ask him or her to clarify.

34. I understand articles I see on how to be healthy.

35. I often feel my time with healthcare providers was not long enough. 
36. When I do not know a word someone uses in talking about health, I ask him or her to explain.

37. I leave interactions with healthcare providers feeling very informed about my health.

38. I understand most of the words people use when they talk about health.

39. I am motivated to listen to a healthcare provider when he or she is talking to me.

40. When healthcare providers tell me how to treat myself at home, I understand how to do so.

41. I am motivated to seek information which will improve my health.

42. I ask healthcare providers questions when I need to.

43. I am motivated to speak with individuals who can help me improve my health.

44. When I do not understand something a healthcare provider has said, I ask him or her to explain it.

45. I have no motivation to talk to others to find information which will improve my health.

46. I understand what others are saying when they give me suggestions on how to improve my health.

47. I am motivated to look for information on how to improve my health.

48. I do not feel that I am able to understand messages about my own health.

49. I am interested in speaking with people who can help me live a healthy life.

50. I am able to understand discussions about health and healthy living.

51. I am able to seek information from others on how to improve my health.

52. When I talk to a healthcare provider about my health, I am able to understand how I can improve my health.

53. I am able to gain access to health information to improve my health.

54. When I talk to a healthcare provider about my health, I am able to understand how to maintain my good health.

55. I am not sure how to find good health-related information.

56. I am able to understand information on how to live a healthy lifestyle.

57. I am not sure which sources are credible when looking for health-related information.

58. I want to understand as much information about my health as possible.

59. I am able to gain access to healthcare when I need it.

60. I am okay with not knowing everything a healthcare provider is talking about with regard to my health.

61. I am able to speak to others who can help me with my health. 
62. When I do not understand information about my health, I do not pay attention to it.

63. I am able to see a doctor or healthcare provider when I need to.

64. I am motivated to understand as much about my health as I am able to.

65. I seek information which helps me maintain a healthy lifestyle.

66. I don't care to understand all of the words a healthcare provider uses during my visit.

67. I speak with others who can help me improve my health.

68. I am interested in understanding how to maintain a healthy lifestyle.

69. I seek information to help me improve my health.

70. I talk to a healthcare provider when I am sick.

71. I visit a doctor or healthcare provider when I need to.

Please answer the following questions describing yourself. This information will be used for analysis only and will not be used to identify you in any way.

Age:

Sex: Male / Female (circle one)

Please indicate your marital status (circle one):

Single Engaged or in a committed relationship Married

Remarried Divorced Widowed

Please indicate how many children you have:

Ethnicity (please circle one - your dominant ethnic background):

Asian Black/African-American Hispanic

Native American White/Caucasian Middle Eastern

Other (specify)

Please indicate your yearly household income (circle one):

0- $\$ 10,000$

$\$ 10,000-\$ 20,000$

$\$ 20,001-\$ 40,000$ 
$\$ 40,001-\$ 60,000$

$\$ 60,001-\$ 80,000$

$\$ 80,001-\$ 100,000$

$\$ 100,0000+$

Please indicate your highest education level (circle one):

Did not finish high school

High school diploma

College degree

Master's degree

Doctorate degree

Professional/trade certification

Thank you for your participation! 


\section{Appendix G}

\section{Item Pool Used in Study One}

1. I feel confident I am able to apply the medical instructions given to me by a healthcare provider when I leave his or her office.

2. I use the health information I see and hear in reference to my own life.

3. I feel confident in my ability to ask a healthcare provider questions.

4. When people I see as credible give me suggestions on how to improve my health, I use the suggestions.

5. I understand the medical instructions healthcare providers give to me.

6. I use health information to my own life and health choices when possible.

7. When I leave an interaction with a healthcare provider, I am confident that I can remember the key points of his or her message.

8. I use the advice my doctor gives to me on how to improve my health.

9. I want to understand the directions given to me by a healthcare provider.

10. I often use "tips" I hear on how to live a healthy life.

11. I directly ask healthcare providers questions.

12. In general, I am able to use the health information I receive.

13. When I have a question for a healthcare provider, I make sure I ask it.

14. I have a hard time using suggestions I hear on how o live a healthy lifestyle.

15. I worry that healthcare providers will think my questions are "dumb."

16. I am not able to figure out how to use the instructions a healthcare provider gives me once I am home.

17. When a healthcare provider is talking to me, I am able to understand most of what he or she is saying.

18. When I hear information on how to improve my health or live a healthy life, I am able to use it.

19. When I leave a healthcare provider's office, I remember exactly what he or she told me to do.

20. I am able to use the suggestions I receive from others on how to improve my health.

21. I am able to listen to a healthcare provider when he or she is talking.

22. I do not use the health suggestions others give me to improve my health.

23. I fully listen to a healthcare provider when he or she is talking.

24. I am not interested in using the health information that I see every day.

25 . When I ask a healthcare provider a question, he or she fully listens to me.

26. I am motivated to use the advice my healthcare provider gives me about my health.

27. I often leave a healthcare providers' office feeling confused.

28. I am interested in using messages I receive from others on how to improve my health.

29. When I leave a healthcare provider's office, I feel like I have said everything I wanted to say.

30. I want to use the health information I see every day to live a healthy life.

31. I understand the terms a healthcare provider uses in interactions with me.

32. I am motivated to use information that I receive to improve my health. 
33. If I do not understand a term that a healthcare provider has used, I do not hesitate to ask him or her to clarify.

34. I understand articles I see on how to be healthy.

35. I often feel that my time with healthcare providers was not long enough.

36. When I do not understand a word someone uses when talking about health, I ask him or her to explain.

37. I leave interactions with healthcare providers feeling very informed about my health.

38. I understand most of the words people use when they talk about health.

39. I am motivated to listen to a healthcare provider when he or she is talking to me.

40. When healthcare providers tell me how to treat myself at home (e.g., take medications), I understand how to do so.

41. I am motivated to seek information which will improve my health.

42. I ask healthcare providers questions when I need to understand things better.

43. I am motivated seek information from people who can help me improve my health.

44. When I do not understand something a healthcare provider has said, I ask him or her to explain it.

45. I have no motivation to seek information from people who can help me improve my health.

46. When people give me suggestions on how to improve my health, I understand what they are trying to tell me and why.

47. I am not motivated to seek information on how to improve my health.

48. I am not able to understand messages about my own health.

49. I am interested in seeking information from people who can help me live a healthy life.

50. I am able to understand discussions about health and healthy living.

51. I know how to seek information from others on how to improve my health.

52. When I talk to a healthcare provider about my health, I am able to understand how I can improve my health.

53. I am able to seek health information to improve my health if I want to.

54. When I talk to a healthcare provider about my health, I am able to understand how to maintain good health.

55. I am not sure how to seek good health-related information.

56. I am able to understand information on how to live a healthy lifestyle.

57. There is so much health information out there, it is hard to tell what is "good information" when I am seeking it.

58. I want to understand as much information about my health as possible.

59. I am able to seek healthcare (e.g., talk to a doctor) when I need it.

60. I am okay with not understanding everything a healthcare provider is talking about regarding my health.

61. I know how to seek out others who can help me with my health.

62. When I do not understand information about my health, I do not pay attention to it.

63. I am able to seek information from a doctor or healthcare provider when I need to.

64. I am motivated to understand information that will help me live a healthy life.

65. I seek information which helps me maintain a healthy lifestyle.

66. It is okay with me to not understand all of the words a healthcare provider uses during my visit. 
67. I don't need to understand all of the words a healthcare provider uses during my visit. 68. I seek out friends who can help me improve my health.

69. I seek out family who can help me improve my health.

70. I seek out others who can help me improve my health.

71. I am interested in understanding how to maintain a healthy lifestyle.

72. I seek information to help me improve my health.

73. I seek a doctor or healthcare provider when I need to.

74. I seek out a healthcare provider when I am sick. 


\section{Appendix $\mathrm{H}$}

\section{Study One Recruitment Script}

Hello class. I am a PhD candidate in the Department of Communication Studies. In order to fulfill requirements for my degree, I am conducting an IRB approved research (IRB protocol \# 1310118439) study under the supervision of Dr. Keith Weber. As [instructor name here] mentioned earlier this week, I am investigating perceptions of individual's motivation and ability to seek, understand, and use health-related information.

This study is completely voluntary and anyone who is 18 years of age or older can participate. Participation entails completing a written survey questionnaire in class, which should take approximately 20 minutes. This is a two part study. For the entire study, you will be asked to complete two questionnaires of similar length and content. I will come back next week to ask you to participate in the second half of the study. You may discontinue your participation at any time without penalty. Your refusal to participate or withdrawal from this study will in no way affect your class standing, grades, job status, or status in any athletic or other activity associated with West Virginia University. There are no known risks to participating in this study.

If you have any additional questions regarding your rights as a research participant or the research study, please feel free to contact me at slabelle@mix.wvu.edu, or come see me in Armstrong 113. Thank you very much for your time. 


\section{Appendix I}

\section{Study One Cover Letter}

\section{WestVhrginiaUniversity}

November 2013

Dear Participant:

This letter is a request for you to take part in a research study to assess perceptions of individual's motivation and ability to seek, understand, and use health-related information. This research study is being conducted by Principal Investigator Dr. Keith Weber, Professor of Communication Studies at West Virginia University, and Co-Investigator Sara LaBelle, Ph.D. Candidate in the Department of Communication Studies at West Virginia University. This research will fulfill requirements toward earning a Ph.D. in Communication Studies for the co-investigator. Your participation is greatly appreciated and will take approximately 20 minutes to fill out the attached questionnaire.

Your responses to the questions on this survey will be strictly confidential, and we will not use anyone's names in written reports. All data will be reported in the aggregate. You must be 18 years of age or older to participate. We will not ask any information that should lead back to your identity as a participant. Your participation is completely voluntary, and you may skip questions you do not wish to answer or discontinue your participation at any time without fear of penalty. Please keep in mind that by handing in this questionnaire, you are agreeing to participate in the study. You do not have to participate if you do not wish to do so. Your refusal to participate or withdrawal from this study will in no way affect your class standing, grades, job status, or status in any athletic or other activity associated with West Virginia University. There are no known risks associated with participation in this study. If you have any questions about your rights as a research participant, you may contact the West Virginia University Institutional Review Board (IRB) at (304) 293-7073. West Virginia University’s Institutional Review Board (IRB) has acknowledgement of this study on file.

We hope that you will participate in this research study, as the results could be beneficial in helping individuals to feel more motivated and able to seek, understand, and use health-related information. Thank you very much for your time. If you would like more information regarding this research project, feel free to contact Principal Investigator Keith Weber at 304-293-3905 or by email at kaweber98@yahoo.com.

Thank you for your help with this research study.

Sincerely,

Dr. Keith Weber

Professor

Principal Investigator

kaweber98@yahoo.com
Sara LaBelle

Ph.D. Candidate

Co-Investigator

slabelle@mix.wvu.edu
Phone: $304-293-3905$

Fax: 304-293.8667
108 Armstrong Hall

PO Box 6293

Morgantown, WV 26506-6293 


\section{Appendix $\mathrm{J}$}

\section{Study One Questionnaire}

The following series of statements reflect your experiences with the information you receive every day regarding your health. This might include, but is not limited to, articles you read in the newspaper or in magazines, articles on the internet, news stories on the internet or on television, and even the conversations you have with friends, family, and healthcare providers on how to improve and maintain good health. Healthcare providers may include your general physician, a health specialist whom you see on a regular basis, a nurse, or even individuals you see in urgent care situations.

\begin{tabular}{|c|c|c|c|c|}
\hline $\begin{array}{c}\text { Strongly } \\
\text { Disagree }\end{array}$ & Disagree & $\begin{array}{c}\text { Neither } \\
\text { Disagree nor } \\
\text { Agree }\end{array}$ & Agree & Strongly Agree \\
\hline 1 & 2 & 3 & 4 & 5 \\
\hline
\end{tabular}

\section{Please take a moment to reflect on the health information you receive from these}

sources. Then, indicate in the space provided how much you agree with each of the following statements using the scale below.

1. I feel confident I am able to apply the medical instructions given to me by a healthcare provider when I leave his or her office.

2. I use the health information I see and hear in reference to my own life.

3. I feel confident in my ability to ask a healthcare provider questions.

4. When people I see as credible give me suggestions on how to improve my health, I use the suggestions.

5. I understand the medical instructions healthcare providers give to me.

6. I use health information to my own life and health choices when possible.

7. When I leave an interaction with a healthcare provider, I am confident that I can remember the key points of his or her message.

8. I use the advice my doctor gives to me on how to improve my health.

9. I want to understand the directions given to me by a healthcare provider.

10. I often use "tips" I hear on how to live a healthy life.

11. I directly ask healthcare providers questions. 


\begin{tabular}{|c|c|c|c|c|}
\hline $\begin{array}{c}\text { Strongly } \\
\text { Disagree }\end{array}$ & Disagree & $\begin{array}{c}\text { Neither } \\
\text { Disagree nor } \\
\text { Agree }\end{array}$ & Agree & Strongly Agree \\
\hline 1 & 2 & 3 & 4 & 5 \\
\hline
\end{tabular}

12. In general, I am able to use the health information I receive.

13. When I have a question for a healthcare provider, I make sure I ask it.

14. I have a hard time using suggestions I hear on how to live a healthy lifestyle.

15. I worry that healthcare providers will think my questions are "dumb.”

16. I am not able to figure out how to use the instructions a healthcare provider gives me once I am home.

17. When a healthcare provider is talking to me, I am able to understand most of what he or she is saying.

18. When I hear information on how to improve my health or live a healthy life, I am able to use it.

19. When I leave a healthcare provider's office, I remember exactly what he or she told me to do.

20. I am able to use the suggestions I receive from others on how to improve my health.

21. I am able to listen to a healthcare provider when he or she is talking.

22. I do not use the health suggestions others give me to improve my health.

23. I fully listen to a healthcare provider when he or she is talking.

24. I am not interested in using the health information that I see every day.

25. When I ask a healthcare provider a question, he or she fully listens to me.

26. I am motivated to use the advice my healthcare provider gives me about my health.

27. I often leave a healthcare providers' office feeling confused.

28. I am interested in using messages I receive from others on how to improve my health.

29. When I leave a healthcare provider's office, I feel like I have said everything I wanted to say.

30. I want to use the health information I see every day to live a healthy life.

31. I understand the terms a healthcare provider uses in interactions with me. 


\begin{tabular}{|c|c|c|c|c|}
\hline $\begin{array}{c}\text { Strongly } \\
\text { Disagree }\end{array}$ & Disagree & $\begin{array}{c}\text { Neither } \\
\text { Disagree nor } \\
\text { Agree }\end{array}$ & Agree & Strongly Agree \\
\hline 1 & 2 & 3 & 4 & 5 \\
\hline
\end{tabular}

32. I am motivated to use information that I receive to improve my health.

33. If I do not understand a term that a healthcare provider has used, I do not hesitate to ask him or her to clarify.

34. I understand articles I see on how to be healthy.

35. I often feel that my time with healthcare providers was not long enough.

36. When I do not understand a word someone uses when talking about health, I ask him or her to explain.

37. I leave interactions with healthcare providers feeling very informed about my health.

38. I understand most of the words people use when they talk about health.

39. I am motivated to listen to a healthcare provider when he or she is talking to me.

40. When healthcare providers tell me how to treat myself at home (e.g., take medications), I understand how to do so.

41. I am motivated to seek information which will improve my health.

42. I ask healthcare providers questions when I need to understand things better.

43. I am motivated seek information from people who can help me improve my health.

44. When I do not understand something a healthcare provider has said, I ask him or her to explain it.

45. I have no motivation to seek information from people who can help me improve my health.

46. When people give me suggestions on how to improve my health, I understand what they are trying to tell me and why.

47. I am not motivated to seek information on how to improve my health.

48. I am not able to understand messages about my own health.

49. I am interested in seeking information from people who can help me live a healthy life. 


\begin{tabular}{|c|c|c|c|c|}
\hline $\begin{array}{c}\text { Strongly } \\
\text { Disagree }\end{array}$ & Disagree & $\begin{array}{c}\text { Neither } \\
\text { Disagree nor } \\
\text { Agree }\end{array}$ & Agree & Strongly Agree \\
\hline 1 & 2 & 3 & 4 & 5 \\
\hline
\end{tabular}

50. I am able to understand discussions about health and healthy living.

51. I know how to seek information from others on how to improve my health.

52. When I talk to a healthcare provider about my health, I am able to understand how I can improve my health.

53. I am able to seek health information to improve my health if I want to.

54. When I talk to a healthcare provider about my health, I am able to understand how to maintain good health.

55. I am not sure how to seek good health-related information.

56. I am able to understand information on how to live a healthy lifestyle.

57. There is so much health information out there, it is hard to tell what is "good information” when I am seeking it.

58. I want to understand as much information about my health as possible.

59. I am able to seek healthcare (e.g., talk to a doctor) when I need it.

60. I am okay with not understanding everything a healthcare provider is talking about regarding my health.

61. I know how to seek out others who can help me with my health.

62. When I do not understand information about my health, I do not pay attention to it.

63. I am able to seek information from a doctor or healthcare provider when I need to.

64. I am motivated to understand information that will help me live a healthy life. 65. I seek information which helps me maintain a healthy lifestyle.

66. It is okay with me to not understand all of the words a healthcare provider uses during my visit.

67. I don't need to understand all of the words a healthcare provider uses during my visit.

68. I seek out friends who can help me improve my health. 


\begin{tabular}{|c|c|c|c|c|}
\hline $\begin{array}{c}\text { Strongly } \\
\text { Disagree }\end{array}$ & Disagree & $\begin{array}{c}\text { Neither } \\
\text { Disagree nor } \\
\text { Agree }\end{array}$ & Agree & Strongly Agree \\
\hline 1 & 2 & 3 & 4 & 5 \\
\hline
\end{tabular}

69. I seek out family who can help me improve my health.

70. I seek out others who can help me improve my health.

71. I am interested in understanding how to maintain a healthy lifestyle.

72. I seek information to help me improve my health.

73. I seek a doctor or healthcare provider when I need to.

74. I seek out a healthcare provider when I am sick.

Please answer the following questions describing yourself. This information will be used for analysis only and will not be used to identify you in any way.

Age:

Sex: Male / Female (circle one)

\section{Class Rank:}

First Year Sophomore Junior Senior Graduate Other

Ethnicity (please circle one - your dominant ethnic background):

Asian Black/African-American Hispanic

Native American White/Caucasian Middle Eastern

Other (specify) 


\section{Appendix K}

\section{Revised Perceived Oral Health Literacy Scale}

The following series of statements reflect your experiences with the information you receive every day regarding your health. This might include, but is not limited to, articles that you read in the newspaper or in magazines, articles on the internet, news stories on the internet or on television, and even the conversations that you have with friends, family, and healthcare providers on how to improve and maintain good health. Healthcare providers may include your general physician, a health specialist that you see on a regular basis, a nurse, or even individuals you see in urgent care situations.

\begin{tabular}{|c|c|c|c|c|}
\hline $\begin{array}{c}\text { Strongly } \\
\text { Disagree }\end{array}$ & Disagree & $\begin{array}{c}\text { Neither } \\
\text { Disagree nor } \\
\text { Agree }\end{array}$ & Agree & Strongly Agree \\
\hline 1 & 2 & 3 & 4 & 5 \\
\hline
\end{tabular}

Please take a moment to reflect on the health information that you receive from these sources. Then, indicate in the space provided how much you agree with each of the following statements using the scale below.

1. I often use "tips" I hear on how to live a healthy life.

2. I want to use the health information I see every day to live a healthy life.

3. I am motivated to use information that I receive to improve my health.

4. I am motivated to seek information which will improve my health.

5. I am motivated to seek information from people who can help me improve my health.

6. I am interested in seeking information from people who can help me live a healthy life.

7. I am motivated to understand information that will help me live a healthy life.

8. I seek information which helps me maintain a healthy lifestyle.

9. I am interested in understanding how to maintain a healthy lifestyle.

10. I seek information to help me improve my health.

11. I am okay with not understanding everything a healthcare provider is talking about regarding my health. (Recoded) 
12. It is okay with me to not understand all of the words a healthcare provider uses during my visit. (Recoded)

13. I don't need to understand all of the words a healthcare provider uses during my visit. (Recoded)

14. I understand the terms a healthcare provider uses in interactions with me.

15. I understand articles I see on how to be healthy.

16. I understand most of the words people use when they talk about health.

17. When healthcare providers tell me how to treat myself at home (e.g., take medications), I understand how to do so.

18. When people give me suggestions on how to improve my health, I understand what they are trying to tell me and why.

19. I am able to understand discussions about health and healthy living.

20. I know how to seek information from others on how to improve my health.

21. When I talk to a healthcare provider about my health, I am able to understand how I can improve my health.

22. When I talk to a healthcare provider about my health, I am able to understand how to maintain good health.

23. I have a hard time using suggestions I hear on how to live a healthy lifestyle. (Recoded)

24. I worry that healthcare providers will think my questions are "dumb.” (Recoded)

25. I am not able to figure out how to use the instructions a healthcare provider gives me once I am home. (Recoded)

26. I do not use the health suggestions others give me to improve my health. (Recoded) 


\section{Appendix L}

\section{Study Two Phase One Recruitment Script}

Hello class. I am a PhD candidate in the Department of Communication Studies. In order to fulfill requirements for my degree, I am conducting an IRB approved research (IRB protocol \#1311136321) study under the supervision of Dr. Keith Weber. As [instructor name here] mentioned earlier this week, I am investigating perceptions of individual's motivation and ability to seek, understand, and use health-related information.

This study is completely voluntary and anyone who is 18 years of age or older can participate. Participation entails completing a written survey questionnaire in class, which should take approximately 20 minutes. This is a two part study. For the entire study, you will be asked to complete two questionnaires of similar length and content. I will come back next week to ask you to participate in the second half of the study. You may discontinue your participation at any time without penalty. Your refusal to participate or withdrawal from this study will in no way affect your class standing, grades, job status, or status in any athletic or other activity associated with West Virginia University. There are no known risks to participating in this study.

If you have any additional questions regarding your rights as a research participant or the research study, please feel free to contact me at slabelle@mix.wvu.edu, or come see me in Armstrong 113. Thank you very much for your time. 


\section{Appendix M}

\section{Study Two Phase One Cover Letter}

\section{West VirginiaUniversity}

January 2014

Dear Participant:

This letter is a request for you to take part in a research study to assess perceptions of individual's motivation and ability to seek, understand, and use health-related information. This research study is being conducted by Principal Investigator Dr. Keith Weber, Professor of Communication Studies at West Virginia University, and Co-Investigator Sara LaBelle, Ph.D. Candidate in the Department of Communication Studies at West Virginia University. This research will fulfill requirements toward earning a Ph.D. in Communication Studies for the co-investigator. Your participation is greatly appreciated and will take approximately 20 minutes to fill out the attached questionnaire. This is a two part study. For the entire study, you will be asked to complete two questionnaires of similar length and content.

Your responses to the questions on this survey will be strictly confidential, and we will not use anyone's names in written reports. All data will be reported in the aggregate. You must be 18 years of age or older to participate. We will not ask any information that should lead back to your identity as a participant. Your participation is completely voluntary, and you may skip questions you do not wish to answer or discontinue your participation at any time without fear of penalty. Please keep in mind that by handing in this questionnaire, you are agreeing to participate in the study. You do not have to participate if you do not wish to do so. Your refusal to participate or withdrawal from this study will in no way affect your class standing, grades, job status, or status in any athletic or other activity associated with West Virginia University. There are no known risks associated with participation in this study. If you have any questions about your rights as a research participant, you may contact the West Virginia University Institutional Review Board (IRB) at (304) 293-7073. West Virginia University's Institutional Review Board (IRB) has acknowledgement of this study on file.

We hope that you will participate in this research study, as the results could be beneficial in helping individuals to feel more motivated and able to seek, understand, and use health-related information. Thank you very much for your time. If you would like more information regarding this research project, feel free to contact Principal Investigator Keith Weber at 304-293-3905 or by email at kaweber98@yahoo.com.

Thank you for your help with this research study.

Sincerely,

Dr. Keith Weber

Professor

Principal Investigator

kaweber98@yahoo.com
Sara LaBelle

Ph.D. Candidate

Co-Investigator

slabelle@mix.wvu.edu
Phone: 304-293-3905

Fax: 304-293-8667
108 Armstrong Hall

PO Box 6293

Morgantown, WV 26506-6293 


\section{Appendix N}

Notes for Educational Intervention

Experimental Group

Lesson on Health Literacy

- Definition of Health Literacy

- Why is it important to be healthy? Why should you care about health information?

- Prevalence and Consequences of Low Health Literacy

- Practical Advice for Being Health Literate

-How can you prevent these things from happening to you?

- $\quad$ Tips for Patients (taken from duPre, 2014)

- "Where can I go if I am sick and need to see a doctor?”

- "What should I ask a doctor when I am there?"

• "Where can I go if I have a question about my health?”

- "Where can I go for helpful tips on how to be healthy and fit?”

\section{Control Group}

Overview of Communication Research

- Defining Research

- The Scientific Method

- Why do Communication Studies research?

1. There are important questions that need to be answered in society.

2. Many important questions lack sufficient answers.

3. Research gives us better answers than just opinions or guessing.

- Four Areas that we Research in Communication Studies 
- Conclusion: What you should be doing now. 


\section{Appendix O}

\section{Study Two Phase Two Recruitment Script}

Hello class. I am a PhD candidate in the Department of Communication Studies. In order to fulfill requirements for my degree, I am conducting an IRB approved research (IRB protocol \#1311136321) study under the supervision of Dr. Keith Weber. As [instructor name here] mentioned earlier this week, I am investigating perceptions of individual's motivation and ability to seek, understand, and use health-related information.

This study is completely voluntary and anyone who is 18 years of age or older can participate. Participation entails completing a written survey questionnaire in class, which should take approximately 20 minutes. This is the second half of the two part study I announced last week. This questionnaire is of similar length and content. You may discontinue your participation at any time without penalty. Your refusal to participate or withdrawal from this study will in no way affect your class standing, grades, job status, or status in any athletic or other activity associated with West Virginia University. There are no known risks to participating in this study.

If you have any additional questions regarding your rights as a research participant or the research study, please feel free to contact me at slabelle@mix.wvu.edu, or come see me in Armstrong 113. Thank you very much for your time. 


\section{Appendix P}

\section{Study Two Phase Two Cover Letter}

\section{WestVirginiaUniversity}

February 2014

Dear Participant:

This letter is a request for you to take part in a research study to assess perceptions of individual's motivation and ability to seek, understand, and use health-related information. This research study is being conducted by Principal Investigator Dr. Keith Weber, Professor of Communication Studies at West Virginia University, and Co-Investigator Sara LaBelle, Ph.D. Candidate in the Department of Communication Studies at West Virginia University. This research will fulfill requirements toward earning a Ph.D. in Communication Studies for the co-investigator. Your participation is greatly appreciated and will take approximately 20 minutes to fill out the attached questionnaire. This is a two part study. For the entire study, you will be asked to complete two questionnaires of similar length and content.

Your responses to the questions on this survey will be strictly confidential, and we will not use anyone's names in written reports. All data will be reported in the aggregate. You must be 18 years of age or older to participate. We will not ask any information that should lead back to your identity as a participant. Your participation is completely voluntary, and you may skip questions you do not wish to answer or discontinue your participation at any time without fear of penalty. Please keep in mind that by handing in this questionnaire, you are agreeing to participate in the study. You do not have to participate if you do not wish to do so. Your refusal to participate or withdrawal from this study will in no way affect your class standing, grades, job status, or status in any athletic or other activity associated with West Virginia University. There are no known risks associated with participation in this study. If you have any questions about your rights as a research participant, you may contact the West Virginia University Institutional Review Board (IRB) at (304) 293-7073. West Virginia University's Institutional Review Board (IRB) has acknowledgement of this study on file.

We hope that you will participate in this research study, as the results could be beneficial in helping individuals to feel more motivated and able to seek, understand, and use health-related information. Thank you very much for your time. If you would like more information regarding this research project, feel free to contact Principal Investigator Keith Weber at 304-293-3905 or by email at kaweber98@yahoo.com.

Thank you for your help with this research study.

Sincerely,

Dr. Keith Weber

Professor

Principal Investigator

kaweber98@yahoo.com
Sara LaBelle

Ph.D. Candidate

Co-Investigator

slabelle@mix.wvu.edu
Phone: $304-293-3905$

Fax: 304-293-8667
PO Box 6293

Morgantown, WV 26506-6293 


\section{Appendix Q}

\section{Perceived Health Competence Measure}

Instructions: Please use the scale below to indicate your agreement to the following set of statements. Write your answer in the space provided. There is neither a right nor a wrong answer.

\begin{tabular}{|c|c|c|c|c|}
\hline $\begin{array}{c}\text { Strongly } \\
\text { Disagree }\end{array}$ & Disagree & $\begin{array}{c}\text { Neither } \\
\text { Disagree nor } \\
\text { Agree }\end{array}$ & Agree & Strongly Agree \\
\hline 1 & 2 & 3 & 4 & 5 \\
\hline
\end{tabular}

1. I handle myself well with respect to my health.

2. No matter how hard I try, my health just doesn't turn out the way I would like. (Recoded)

3. It is difficult for me to find effective solutions to the health problems that come my way. (Recoded)

4. I succeed in the projects I undertake to improve my health.

5. I'm generally able to accomplish my goals with respect to my health.

6. I find my efforts to change things I don't like about my health are ineffective. (Recoded)

7. Typically, my plans for my health don’t work out well. (Recoded)

8. I am able to do things for my health as well as most other people. 
Appendix R

\section{Patient Self and Response Efficacy Measure}

Instructions: With your healthcare provider in mind, please use the scale below to indicate your agreement to the following set of statements. Write your answer in the space provided. There is neither a right nor a wrong answer.

In this study, the term healthcare provider is used to represent the person you usually see for your health needs. This might be a general health practitioner, a specialist, or a nurse. As you answer the questions on this survey, keep in mind the healthcare provider you see most often for your health needs.

\begin{tabular}{|c|c|c|c|c|}
\hline $\begin{array}{c}\text { Strongly } \\
\text { Disagree }\end{array}$ & Disagree & $\begin{array}{c}\text { Neither } \\
\text { Disagree nor } \\
\text { Agree }\end{array}$ & Agree & Strongly Agree \\
\hline 1 & 2 & 3 & 4 & 5 \\
\hline
\end{tabular}

1. I am able to ask my healthcare provider any question regarding my medical condition and treatment.

2. I think it would be easy to participate in medical decision-making with my healthcare provider.

3. It is often difficult for me to express my point of view with my healthcare provider. (Recoded)

4. Open communication with my healthcare provider will help improve the quality of my medical care.

5. I will be better off if I ask questions at any time during my appointment.

6. I will follow instructions better if I agree with the healthcare provider's recommended treatment.

Self Efficacy: 1, 2 and 3

Response Efficacy: 4, 5 and 6 


\section{Appendix S}

Form Trait subscale of the Communication Anxiety Inventory

Instructions: The following are statements that describe various communication events. You are asked to respond to items in terms of how you generally feel about these events. Please mark your response in the appropriate blank on the answer sheet. Be sure to give the response that best describes how you generally feel.

1. I think I communicate effectively in one-to-one situations. (Recoded)

2. My heart beats faster than usual when I speak out in a small group meeting.

3. I enjoy speaking in public. (Recoded)

4. I avoid talking with individuals I don't know very well.

5. I think I make a poor impression when I speak at a small group meeting.

6. I feel disappointed in myself after speaking in public.

7. I enjoy talking with someone I've just met. (Recoded)

8. My body feels relaxed when I speak during a small group meeting. (Recoded)

9. I avoid speaking in public if possible.

10. My body feels tense when I talk with someone I don't know very well.

11. I speak out during small group meetings. (Recoded)

12. I am terrified at the thought of speaking in public.

13. My heart beats faster than usual when I talk to someone I've just met.

14. I enjoy talking at a small group meeting. (Recoded)

15. I make a good impression when I speak in public. (Recoded)

16. I would like to have a job that requires me to talk often on a one-to-one basis.

(Recoded)

17. I feel disappointed in my efforts to communicate at a small group meeting.

18. My body feels tense and stiff when I speak in public.

19. When conversing with someone on a one-to-one basis, I prefer to listen rather

than to talk.

20. I avoid talking during small group meetings.

21. I look forward to speaking in public. (Recoded)

Dyadic: 1, 4, 7, 10, 13, 16, and 19

Small Group: 2, 5, 8, 11, 14, 17, and 20

Public Speaking: 3, 6, 9, 12, 15, 18, and 21 


\section{Appendix T}

Study Two Phase One and Two Questionnaire

\section{6-Digit Code \#: \\ (last 4 digits of your telephone number followed by your 2-digit birth month)}

The following series of statements reflect your experiences with the information you receive every day regarding your health. This might include, but is not limited to, articles you read in the newspaper or in magazines, articles on the internet, news stories on the internet or on television, and even the conversations you have with friends, family, and healthcare providers on how to improve and maintain good health. Healthcare providers may include your general physician, a health specialist whom you see on a regular basis, a nurse, or even individuals you see in urgent care situations.

\section{Please take a moment to reflect on the health information you receive from these sources.}

Then, indicate in the space provided how much you agree with each of the following statements using the scale below.

\begin{tabular}{|c|c|c|c|c|}
\hline $\begin{array}{c}\text { Strongly } \\
\text { Disagree }\end{array}$ & Disagree & $\begin{array}{c}\text { Neither Disagree } \\
\text { nor Agree }\end{array}$ & Agree & Strongly Agree \\
\hline 1 & 2 & 3 & 4 & 5 \\
\hline
\end{tabular}

1. I feel confident I am able to apply the medical instructions given to me by a healthcare provider when I leave his or her office.

2. I use the health information I see and hear in reference to my own life.

3. I feel confident in my ability to ask a healthcare provider questions.

4. When people I see as credible give me suggestions on how to improve my health, I use the suggestions.

5. I understand the medical instructions healthcare providers give to me.

6. I use health information to my own life and health choices when possible.

7. When I leave an interaction with a healthcare provider, I am confident that I can remember the key points of his or her message.

8. I use the advice my doctor gives to me on how to improve my health. 


\begin{tabular}{|c|c|c|c|c|}
\hline $\begin{array}{c}\text { Strongly } \\
\text { Disagree }\end{array}$ & Disagree & $\begin{array}{c}\text { Neither Disagree } \\
\text { nor Agree }\end{array}$ & Agree & Strongly Agree \\
\hline 1 & 2 & 3 & 4 & 5 \\
\hline
\end{tabular}

9. I want to understand the directions given to me by a healthcare provider.

10. I often use "tips" I hear on how to live a healthy life.

11. I directly ask healthcare providers questions.

12. In general, I am able to use the health information I receive.

13. When I have a question for a healthcare provider, I make sure I ask it.

14. I have a hard time using suggestions I hear on how to live a healthy lifestyle.

15. I worry that healthcare providers will think my questions are "dumb."

16. I am not able to figure out how to use the instructions a healthcare provider gives me once I am home.

17. When a healthcare provider is talking to me, I am able to understand most of what he or she is saying.

18. When I hear information on how to improve my health or live a healthy life, I am able to use it.

19. When I leave a healthcare provider's office, I remember exactly what he or she told me to do.

20. I am able to use the suggestions I receive from others on how to improve my health.

21. I am able to listen to a healthcare provider when he or she is talking.

22. I do not use the health suggestions others give me to improve my health.

23. I fully listen to a healthcare provider when he or she is talking.

24. I am not interested in using the health information that I see every day.

25. When I ask a healthcare provider a question, he or she fully listens to me.

26. I am motivated to use the advice my healthcare provider gives me about my health.

27. I often leave a healthcare providers' office feeling confused.

28. I am interested in using messages I receive from others on how to improve my health. 


\begin{tabular}{|c|c|c|c|c|}
\hline $\begin{array}{c}\text { Strongly } \\
\text { Disagree }\end{array}$ & Disagree & $\begin{array}{c}\text { Neither Disagree } \\
\text { nor Agree }\end{array}$ & Agree & Strongly Agree \\
\hline 1 & 2 & 3 & 4 & 5 \\
\hline
\end{tabular}

29. When I leave a healthcare provider's office, I feel like I have said everything I wanted to say.

30. I want to use the health information I see every day to live a healthy life.

31. I understand the terms a healthcare provider uses in interactions with me.

32. I am motivated to use information that I receive to improve my health.

33. If I do not understand a term that a healthcare provider has used, I do not hesitate to ask him or her to clarify.

34. I understand articles I see on how to be healthy.

35. I often feel that my time with healthcare providers was not long enough.

36. When I do not understand a word someone uses when talking about health, I ask him or her to explain.

37. I leave interactions with healthcare providers feeling very informed about my health.

38. I understand most of the words people use when they talk about health.

39. I am motivated to listen to a healthcare provider when he or she is talking to me.

40. When healthcare providers tell me how to treat myself at home (e.g., take medications), I understand how to do so.

41. I am motivated to seek information which will improve my health.

42. I ask healthcare providers questions when I need to understand things better.

43. I am motivated seek information from people who can help me improve my health.

44. When I do not understand something a healthcare provider has said, I ask him or her to explain it.

45. I have no motivation to seek information from people who can help me improve my health.

46. When people give me suggestions on how to improve my health, I understand what they are trying to tell me and why. 


\begin{tabular}{|c|c|c|c|c|}
\hline $\begin{array}{c}\text { Strongly } \\
\text { Disagree }\end{array}$ & Disagree & $\begin{array}{c}\text { Neither Disagree } \\
\text { nor Agree }\end{array}$ & Agree & Strongly Agree \\
\hline 1 & 2 & 3 & 4 & 5 \\
\hline
\end{tabular}

47. I am not motivated to seek information on how to improve my health.

48. I am not able to understand messages about my own health.

49. I am interested in seeking information from people who can help me live a healthy life.

50. I am able to understand discussions about health and healthy living.

51. I know how to seek information from others on how to improve my health.

52. When I talk to a healthcare provider about my health, I am able to understand how I can improve my health.

53. I am able to seek health information to improve my health if I want to.

54. When I talk to a healthcare provider about my health, I am able to understand how to maintain good health.

55. I am not sure how to seek good health-related information.

56. I am able to understand information on how to live a healthy lifestyle.

57. There is so much health information out there, it is hard to tell what is "good information” when I am seeking it.

58. I want to understand as much information about my health as possible.

59. I am able to seek healthcare (e.g., talk to a doctor) when I need it.

60. I am okay with not understanding everything a healthcare provider is talking about regarding my health.

61. I know how to seek out others who can help me with my health.

62. When I do not understand information about my health, I do not pay attention to it.

63. I am able to seek information from a doctor or healthcare provider when I need to.

64. I am motivated to understand information that will help me live a healthy life.

65. I seek information which helps me maintain a healthy lifestyle.

66. It is okay with me to not understand all of the words a healthcare provider uses during my visit. 


\begin{tabular}{|c|c|c|c|c|}
\hline $\begin{array}{c}\text { Strongly } \\
\text { Disagree }\end{array}$ & Disagree & $\begin{array}{c}\text { Neither } \\
\text { Disagree nor } \\
\text { Agree }\end{array}$ & Agree & Strongly Agree \\
\hline 1 & 2 & 3 & 4 & 5 \\
\hline
\end{tabular}

67. I don't need to understand all of the words a healthcare provider uses during my visit.

68. I seek out friends who can help me improve my health.

69. I seek out family who can help me improve my health.

70. I seek out others who can help me improve my health.

71. I am interested in understanding how to maintain a healthy lifestyle.

72. I seek information to help me improve my health.

73. I seek a doctor or healthcare provider when I need to.

74. I seek out a healthcare provider when I am sick.

Instructions: The following are statements that describe various communication events. You are asked to respond to items in terms of how you generally feel about these events. Please mark your response in the appropriate blank on the answer sheet. Be sure to give the response that best describes how you generally feel.

\begin{tabular}{|c|c|c|c|c|}
\hline $\begin{array}{c}\text { Strongly } \\
\text { Disagree }\end{array}$ & Disagree & $\begin{array}{c}\text { Neither } \\
\text { Disagree nor } \\
\text { Agree }\end{array}$ & Agree & Strongly Agree \\
\hline 1 & 2 & 3 & 4 & 5 \\
\hline
\end{tabular}

1. I think I communicate effectively in one-to-one situations.

2. My heart beats faster than usual when I speak out in a small group meeting.

3. I enjoy speaking in public.

4. I avoid talking with individuals I don't know very well.

5. I think I make a poor impression when I speak at a small group meeting.

6. I feel disappointed in myself after speaking in public.

7. I enjoy talking with someone I've just met.

8. My body feels relaxed when I speak during a small group meeting. 


\begin{tabular}{|c|c|c|c|c|}
\hline $\begin{array}{c}\text { Strongly } \\
\text { Disagree }\end{array}$ & Disagree & $\begin{array}{c}\text { Neither Disagree } \\
\text { nor Agree }\end{array}$ & Agree & Strongly Agree \\
\hline 1 & 2 & 3 & 4 & 5 \\
\hline
\end{tabular}

9. I avoid speaking in public if possible.

10. My body feels tense when I talk with someone I don't know very well.

11. I speak out during small group meetings.

12. I am terrified at the thought of speaking in public.

13. My heart beats faster than usual when I talk to someone I've just met.

14. I enjoy talking at a small group meeting.

15. I make a good impression when I speak in public.

16. I would like to have a job that requires me to talk often on a one-to-one basis.

17. I feel disappointed in my efforts to communicate at a small group meeting.

18. My body feels tense and stiff when I speak in public.

19. When conversing with someone on a one-to-one basis, I prefer to listen rather than to talk.

20. I avoid talking during small group meetings.

21. I look forward to speaking in public.

22. I handle myself well with respect to my health.

23. No matter how hard I try, my health just doesn't turn out the way I would like.

24. It is difficult for me to find effective solutions to the health problems that come my way.

25. I succeed in the projects I undertake to improve my health.

26. I'm generally able to accomplish my goals with respect to my health.

27. I find my efforts to change things I don't like about my health are ineffective.

28. Typically, my plans for my health don't work out well.

29. I am able to do things for my health as well as most other people. 
Instructions: With your healthcare provider in mind, please use the scale below to indicate your agreement to the following set of statements. Write your answer in the space provided. There is neither a right nor a wrong answer.

\begin{tabular}{|c|c|c|c|c|}
\hline $\begin{array}{c}\text { Strongly } \\
\text { Disagree }\end{array}$ & Disagree & $\begin{array}{c}\text { Neither } \\
\text { Disagree nor } \\
\text { Agree }\end{array}$ & Agree & Strongly Agree \\
\hline 1 & 2 & 3 & 4 & 5 \\
\hline
\end{tabular}

In this study, the term healthcare provider is used to represent the person you usually see for your health needs. This might be a general health practitioner, a specialist, or a nurse. As you answer the questions on this survey, keep in mind the healthcare provider you see most often for your health needs.

1. I am able to ask my healthcare provider any question regarding my medical condition and treatment.

2. I think it would be easy to participate in medical decision-making with my healthcare provider.

3. It is often difficult for me to express my point of view with my healthcare provider.

4. Open communication with my healthcare provider will help improve the quality of my medical care.

5. I will be better off if I ask questions at any time during my appointment.

6. I will follow instructions better if I agree with the healthcare provider's recommended treatment.

Please answer the following questions describing yourself. This information will be used for analysis only and will not be used to identify you in any way.

Age:

Sex: Male / Female (circle one)

Class Rank (circle one):

First Year Sophomore Junior Senior Graduate Other 
Ethnicity (please circle one - your dominant ethnic background):

Asian

Native American

Other (specify)
Black/African-American Hispanic

White/Caucasian

Middle Eastern

Thank you for your participation! 


\section{Appendix U}

\section{Study Three Recruitment Script}

Hello class. I am a PhD candidate in the Department of Communication Studies. In order to fulfill requirements for my degree, I am conducting an IRB approved research (IRB protocol \# 1310118775) study under the supervision of Dr. Keith Weber. As [instructor name here] mentioned earlier this week, I am investigating perceptions of individual's motivation and ability to seek, understand, and use health-related information.

This study is completely voluntary and anyone who is 18 years of age or older can participate. Participation entails completing a written survey questionnaire in class, which should take approximately 20 minutes. This is a two part study. For the entire study, you will be asked to complete two questionnaires of similar length and content. I will come back next week to ask you to participate in the second half of the study. You may discontinue your participation at any time without penalty. Your refusal to participate or withdrawal from this study will in no way affect your class standing, grades, job status, or status in any athletic or other activity associated with West Virginia University. There are no known risks to participating in this study.

If you have any additional questions regarding your rights as a research participant or the research study, please feel free to contact me at slabelle@mix.wvu.edu, or come see me in Armstrong 113. Thank you very much for your time. 
Appendix V

\section{Study Three Cover Letter}

\section{West VirginiaUniversity}

Department of Communication Studies

November 2013

Dear Participant:

This letter is a request for you to take part in a research study to assess perceptions of individual's motivation and ability to seek, understand, and use health-related information. This research study is being conducted by Principal Investigator Dr. Keith Weber, Professor of Communication Studies at West Virginia University, and Co-Investigator Sara LaBelle, Ph.D. Candidate in the Department of Communication Studies at West Virginia University. This research will fulfill requirements toward earning a Ph.D. in Communication Studies for the co-investigator. Your participation is greatly appreciated and will take approximately 20 minutes to fill out the attached questionnaire.

Your responses to the questions on this survey will be strictly confidential, and we will not use anyone's names in written reports. All data will be reported in the aggregate. You must be 18 years of age or older to participate. We will not ask any information that should lead back to your identity as a participant. Your participation is completely voluntary, and you may skip questions you do not wish to answer or discontinue your participation at any time without fear of penalty. Please keep in mind that by handing in this questionnaire, you are agreeing to participate in the study. You do not have to participate if you do not wish to do so. Your refusal to participate or withdrawal from this study will in no way affect your class standing, grades, job status, or status in any athletic or other activity associated with West Virginia University. There are no known risks associated with participation in this study. If you have any questions about your rights as a research participant, you may contact the West Virginia University Institutional Review Board (IRB) at (304) 293-7073. West Virginia University's Institutional Review Board (IRB) has acknowledgement of this study on file.

We hope that you will participate in this research study, as the results could be beneficial in helping individuals to feel more motivated and able to seek, understand, and use health-related information. Thank you very much for your time. If you would like more information regarding this research project, feel free to contact Principal Investigator Keith Weber at 304-293-3905 or by email at kaweber98@yahoo.com.

Thank you for your help with this research study.

Sincerely,

Dr. Keith Weber

Professor

Principal Investigator

kaweber98@yahoo.com
Sara LaBelle

Ph.D. Candidate

Co-Investigator

slabelle@mix.wvu.edu

108 Armstrong Hall

PO Box 6293 


\section{Appendix W}

\section{Study Three Questionnaire}

The following series of statements reflect your experiences with the information you receive every day regarding your health. This might include, but is not limited to, articles you read in the newspaper or in magazines, articles on the internet, news stories on the internet or on television, and even the conversations you have with friends, family, and healthcare providers on how to improve and maintain good health. Healthcare providers may include your general physician, a health specialist whom you see on a regular basis, a nurse, or even individuals you see in urgent care situations.

Please take a moment to reflect on the health information you receive from these sources. Then, indicate in the space provided how much you agree with each of the following statements using the scale below.

\begin{tabular}{|c|c|c|c|c|}
\hline $\begin{array}{c}\text { Strongly } \\
\text { Disagree }\end{array}$ & Disagree & $\begin{array}{c}\text { Neither } \\
\text { Disagree nor } \\
\text { Agree }\end{array}$ & Agree & Strongly Agree \\
\hline 1 & 2 & 3 & 4 & 5 \\
\hline
\end{tabular}

1. I feel confident I am able to apply the medical instructions given to me by a healthcare provider when I leave his or her office.

2. I use the health information I see and hear in reference to my own life.

3. I feel confident in my ability to ask a healthcare provider questions.

4. When people I see as credible give me suggestions on how to improve my health, I use the suggestions.

5. I understand the medical instructions healthcare providers give to me.

6. I use health information to my own life and health choices when possible.

7. When I leave an interaction with a healthcare provider, I am confident that I can remember the key points of his or her message.

8. I use the advice my doctor gives to me on how to improve my health.

9. I want to understand the directions given to me by a healthcare provider. 


\begin{tabular}{|c|c|c|c|c|}
\hline $\begin{array}{c}\text { Strongly } \\
\text { Disagree }\end{array}$ & Disagree & $\begin{array}{c}\text { Neither Disagree } \\
\text { nor Agree }\end{array}$ & Agree & Strongly Agree \\
\hline 1 & 2 & 3 & 4 & 5 \\
\hline
\end{tabular}

10. I often use "tips" I hear on how to live a healthy life.

11. I directly ask healthcare providers questions.

12. In general, I am able to use the health information I receive.

13. When I have a question for a healthcare provider, I make sure I ask it.

14. I have a hard time using suggestions I hear on how to live a healthy lifestyle.

15. I worry that healthcare providers will think my questions are "dumb.”

16. I am not able to figure out how to use the instructions a healthcare provider gives me once I am home.

17. When a healthcare provider is talking to me, I am able to understand most of what he or she is saying.

18. When I hear information on how to improve my health or live a healthy life, I am able to use it.

19. When I leave a healthcare provider's office, I remember exactly what he or she told me to do.

20. I am able to use the suggestions I receive from others on how to improve my health.

21. I am able to listen to a healthcare provider when he or she is talking.

22. I do not use the health suggestions others give me to improve my health.

23. I fully listen to a healthcare provider when he or she is talking.

24. I am not interested in using the health information that I see every day.

25. When I ask a healthcare provider a question, he or she fully listens to me.

26. I am motivated to use the advice my healthcare provider gives me about my health.

27. I often leave a healthcare providers' office feeling confused.

28. I am interested in using messages I receive from others on how to improve my health.

29. When I leave a healthcare provider's office, I feel like I have said everything I wanted to say. 


\begin{tabular}{|c|c|c|c|c|}
\hline $\begin{array}{c}\text { Strongly } \\
\text { Disagree }\end{array}$ & Disagree & $\begin{array}{c}\text { Neither Disagree } \\
\text { nor Agree }\end{array}$ & Agree & Strongly Agree \\
\hline 1 & 2 & 3 & 4 & 5 \\
\hline
\end{tabular}

30. I want to use the health information I see every day to live a healthy life.

31. I understand the terms a healthcare provider uses in interactions with me.

32. I am motivated to use information that I receive to improve my health.

33. If I do not understand a term that a healthcare provider has used, I do not hesitate to ask him or her to clarify.

34. I understand articles I see on how to be healthy.

35. I often feel that my time with healthcare providers was not long enough.

36. When I do not understand a word someone uses when talking about health, I ask him or her to explain.

37. I leave interactions with healthcare providers feeling very informed about my health.

38. I understand most of the words people use when they talk about health.

39. I am motivated to listen to a healthcare provider when he or she is talking to me.

40. When healthcare providers tell me how to treat myself at home (e.g., take medications), I understand how to do so.

41. I am motivated to seek information which will improve my health.

42. I ask healthcare providers questions when I need to understand things better.

43. I am motivated seek information from people who can help me improve my health.

44. When I do not understand something a healthcare provider has said, I ask him or her to explain it.

45. I have no motivation to seek information from people who can help me improve my health.

46. When people give me suggestions on how to improve my health, I understand what they are trying to tell me and why.

47. I am not motivated to seek information on how to improve my health.

48. I am not able to understand messages about my own health. 


\begin{tabular}{|c|c|c|c|c|}
\hline $\begin{array}{c}\text { Strongly } \\
\text { Disagree }\end{array}$ & Disagree & $\begin{array}{c}\text { Neither Disagree } \\
\text { nor Agree }\end{array}$ & Agree & Strongly Agree \\
\hline 1 & 2 & 3 & 4 & 5 \\
\hline
\end{tabular}

49. I am interested in seeking information from people who can help me live a healthy life.

50. I am able to understand discussions about health and healthy living.

51. I know how to seek information from others on how to improve my health.

52. When I talk to a healthcare provider about my health, I am able to understand how I can improve my health.

53. I am able to seek health information to improve my health if I want to.

54. When I talk to a healthcare provider about my health, I am able to understand how to maintain good health.

55. I am not sure how to seek good health-related information.

56. I am able to understand information on how to live a healthy lifestyle.

57. There is so much health information out there, it is hard to tell what is "good information” when I am seeking it.

58. I want to understand as much information about my health as possible.

59. I am able to seek healthcare (e.g., talk to a doctor) when I need it.

60. I am okay with not understanding everything a healthcare provider is talking about regarding my health.

61. I know how to seek out others who can help me with my health.

62. When I do not understand information about my health, I do not pay attention to it.

63. I am able to seek information from a doctor or healthcare provider when I need to.

64. I am motivated to understand information that will help me live a healthy life.

65. I seek information which helps me maintain a healthy lifestyle.

66. It is okay with me to not understand all of the words a healthcare provider uses during my visit.

67. I don't need to understand all of the words a healthcare provider uses during my visit. 
68. I seek out friends who can help me improve my health.

69. I seek out family who can help me improve my health.

70. I seek out others who can help me improve my health.

71. I am interested in understanding how to maintain a healthy lifestyle.

72. I seek information to help me improve my health.

73. I seek a doctor or healthcare provider when I need to.

74. I seek out a healthcare provider when I am sick.

Please answer the following questions describing yourself. This information will be used for analysis only and will not be used to identify you in any way.

Age:

Sex: Male / Female (circle one)

Class Rank:

First Year Sophomore Junior Senior Graduate Other

Ethnicity (please circle one - your dominant ethnic background):

Asian Black/African-American Hispanic

Native American White/Caucasian Middle Eastern

Other (specify) 


\section{Appendix X}

\section{Study Four Recruitment Script}

Hello, my name is Sara LaBelle and I am a Ph.D. candidate in the Department of Communication Studies, and I am conducting a research study with Dr. Keith Weber. This research study is completely voluntary, and West Virginia University's IRB has acknowledged this study. We would like to hear from residents of Monongalia County on their health needs, concerns, and current behaviors so that we (WVU Comm Studies) can create better health programs and services in the future.

I am speaking with you today to see if you are willing to complete a 20 minute written questionnaire on individuals' motivation and ability to gain access to, understand, and use health-related information. In order to fully complete this questionnaire, you will also be asked to provide the results of an A1C test (which we will provide the equipment for). All aspects of this survey are completely voluntary and you may choose to withdraw at any time or not answer any questions that you do not wish to answer. There are no known risks to participants in this study. All of your responses will remain confidential and your responses will not be used to identify you as an individual in any way.

Once again, my name is Sara LaBelle, you can contact me at this number (716 3616116 ) or email me if you choose (slabelle@mix.wvu.edu).

Thank you so much for your time and consideration! 
Appendix Y

Study Four Recruitment Flyer

\section{Free Health Screening $3 \mathrm{pm}$ to $6 \mathrm{pm}$ at The Shack Neighborhood House}

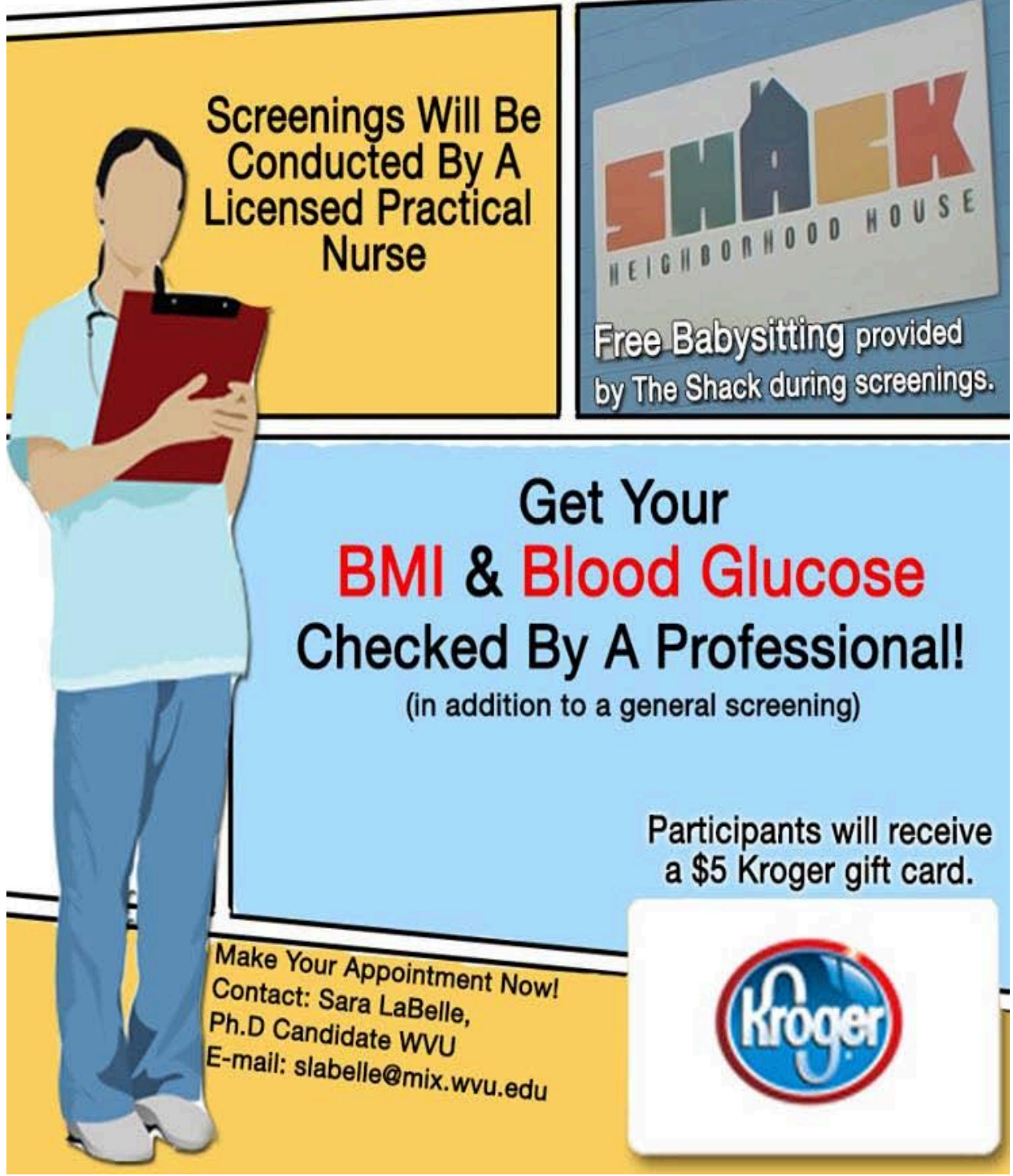


Appendix Z

Study Four Consent Form

WestViroiniaUniversitye

Human Research Protocol

Only Minimal Risk Consent

Form

Without HIPAA

\section{Only Minimal Risk \\ Consent Information Form (without HIPAA)}

$\begin{array}{ll}\text { Principal Investigator } & \text { Keith Weber } \\ \text { Department } & \text { Communication Studies } \\ \text { Protocol Number } & 1312153380 \\ \text { Study Title } & \text { Validation of the Communication Health Literacy Scale in a Community } \\ \text { Context } & \\ \text { Co-Investigator(s) } & \text { Sara LaBelle } \\ \text { Sponsor (if any) } & \text { N/A }\end{array}$

\section{Contact Persons}

In the event you experience any side effects or injury related to this research, you should contact Dr. Keith Weber at (304) 293-3905. (After hours contact: Dr. Keith Weber at (304) 692-9999). If you have any questions, concerns, or complaints about this research, you can contact Dr. Keith Weber or co-investigator Sara LaBelle at (304) 293-3905.

For information regarding your rights as a research subject, to discuss problems, concerns, or suggestions related to the research, to obtain information or offer input about the research, contact the Office of Research Integrity \& Compliance at (304) 293-7073.

In addition if you would like to discuss problems, concerns, have suggestions related to research, or would like to offer input about the research, contact the Office of Research Integrity and Compliance at 304-293-7073.

\section{Introduction}

You, , have been asked to participate in this research study, which has been explained to you by . This study is being conducted by

Dr. Keith Weber and co-investigator Sara LaBelle in the Department of Communication Studies at West Virginia University.

\section{Purpose of the Study}

This study is meant to help validate a recently developed measure of health literacy that can be used by communication studies researchers to assess individuals' motivation and ability to seek, understand, and use 
health information. To do so, we are assessing the relationship of the new measure of health literacy to individuals' level of willingness to prevent diabetes and obesity, as well as indicators of physical health.

\section{Description of Procedures}

This study involves completing a written questionnaire and providing the results of a glucose meter test (which we will provide the equipment for). This will take approximately 20 minutes for you to complete. You will be asked to fill out a questionnaire regarding your motivation and ability to seek, understand, and use health-related information, as well as a few demographic and health-related questions. You do not have to answer all the questions. You will have the opportunity to see the questionnaire before signing this consent form.

\section{Discomforts}

There are no known or expected risks from participating in this study, except for the mild frustration associated with answering the questions.

\section{Alternatives}

You do not have to participate in this study.

\section{Benefits}

You may not receive any direct benefit from this study. The knowledge gained from this study may eventually benefit others.

\section{Financial Considerations}

There are no special fees for participating in this study.

\section{Confidentiality}

Any information about you that is obtained as a result of your participation in this research will be kept as confidential as legally possible. Your research records and test results, just like hospital records, may be subpoenaed by court order or may be inspected by the study sponsor or federal regulatory authorities (including the FDA if applicable) without your additional consent.

In addition, there are certain instances where the researcher is legally required to give information to the appropriate authorities. These would include mandatory reporting of infectious diseases, mandatory reporting of information about behavior that is imminently dangerous to your child or to others, such as suicide, child abuse, etc.

In any publications that result from this research, neither your name nor any information from which you might be identified will be published without your consent.

\section{Voluntary Participation}

Participation in this study is voluntary. You are free to withdraw your consent to participate in this study at any time.

Refusal to participate or withdrawal will involve no penalty to you.

In the event new information becomes available that may affect your willingness to participate in this study, this information will be given to you so that you can make an informed decision about whether or not to continue your participation. 
You have been given the opportunity to ask questions about the research, and you have received answers concerning areas you did not understand.

Upon signing this form, you will receive a copy.

I willingly consent to participate in this research.

\section{Signatures}

Signature of Subject

Printed Name

Date

Time

The participant has had the opportunity to have questions addressed. The participant willingly agrees to be in the study.

Signature of Investigator or Co-Investigator

Printed Name

Date

Time 


\section{Appendix AA}

\section{Study Four Cover Letter}

\section{West VirginiaUniversity}

Department of Communication Studies

February 2014

\section{Dear Participant:}

This letter is a request for you to take part in a research study to assess perceptions of individual's motivation and ability to seek, understand, and use health-related information. This research study is being conducted by Principal Investigator Dr. Keith Weber, Professor of Communication Studies at West Virginia University, and Co-Investigator Sara LaBelle, Ph.D. Candidate in the Department of Communication Studies at West Virginia University. This research will fulfill requirements toward earning a Ph.D. in Communication Studies for the co-investigator. Your participation is greatly appreciated and will take approximately 20 minutes to fill out the attached questionnaire.

Your responses to the questions on this survey will be strictly confidential, and we will not use anyone's names in written reports. You must be 18 years of age or older to participate. We will not ask any information that should lead back to your identity as a participant. Further, the data will not be reported in a way that reveals your identity. In order to fully complete this questionnaire, you will also be asked to provide the results of a glucose meter test (which we will provide the equipment for). Your participation in all parts of the questionnaire is completely voluntary, and you may skip questions you do not wish to answer or discontinue your participation at any time without fear of penalty. Please keep in mind that by handing in this questionnaire, you are agreeing to participate in the study. You do not have to participate if you do not wish to do so. There are no known risks associated with participation in this study. If you have any questions about your rights as a research participant, you may contact the West Virginia University Institutional Review Board (IRB) at (304) 293-7073. West Virginia University's Institutional Review Board (IRB) has acknowledgement of this study on file.

We hope that you will participate in this research study, as the results could be beneficial in helping individuals to feel more motivated and able to seek, understand, and use health-related information. Thank you very much for your time. If you would like more information regarding this research project, feel free to contact Principal Investigator Keith Weber at 304-293-3905 or by email at kaweber98@yahoo.com.

Thank you for your help with this research study.

Sincerely,

' Dr. Keith Weber

Professor

Principal Investigator

kaweber98@yahoo.com
Sara LaBelle Ph.D. Candidate Co-Investigator slabelle@mix.wvu.edu 


\section{Appendix BB}

\section{Stages of Change Measure}

Instructions: The following questions concern your current behaviors related to physical activity and healthy eating. For this study, physical activity includes at least 150 minutes of moderate-intensity aerobic physical activity throughout the week or at least 75 minutes of vigorous-intensity aerobic physical activity throughout the week or an equivalent combination of moderate- and vigorous-intensity activity. Healthy eating refers to a diet consisting of low fat and low calorie foods.

Please select ONE of the five options below to best describe your intentions toward physical activity. Circle your response.

(1) No, I do not do the amount of physical activity indicated above, and I do not intend to in the next six months

(2) No, I do not do the amount of physical activity indicated above, but I intend to in the next six months

(3) No, I do not do the amount of physical activity indicated above, but I intend to in the next 30 days

(4) Yes, I regularly do the amount of physical activity indicated above, but I have been doing so for less than six months

(5) Yes, I do the amount of physical activity indicated above, and I have been doing so for more than six months

Please select ONE of the five options below to best describe your intentions toward healthy eating. Circle your response.

(1) No, I do not eat a diet of low fat and low calorie foods, and I do not intend to in the next six months

(2) No, I do not eat a diet of low fat and low calorie foods, but I intend to in the next six months

(3) No, I do not eat a diet of low fat and low calorie foods, but I intend to in the next 30 days

(4) Yes, I do eat a diet of low fat and low calorie foods, but I have been doing so for less than six months

(5) Yes, I do eat a diet of low fat and low calorie foods, and I have been for more than six months 


\section{Appendix CC}

\section{Physiological Health Indicator Measures}

The following question is an indicator of your overall health. If you know your blood glucose level, please write it in the black. If you do not know this information, you may use the glucose meter provided with assistance from the registered LPN on site. This question, like all of the others in this questionnaire, is voluntary.

Blood Glucose Level:

Body Mass Index: 


\section{Appendix DD}

Study Four Questionnaire

The following series of statements reflect your experiences with the information you receive every day regarding your health. This might include, but is not limited to, articles that you read in the newspaper or in magazines, articles on the internet, news stories on the internet or on television, and even the conversations that you have with friends, family, and healthcare providers on how to improve and maintain good health. Healthcare providers may include your general physician, a health specialist that you see on a regular basis, a nurse, or even individuals you see in urgent care situations.

Please take a moment to reflect on the health information that you receive from these sources. Then, indicate in the space provided how much you agree with each of the following statements using the scale below.

\begin{tabular}{|c|c|c|c|c|}
\hline $\begin{array}{c}\text { Strongly } \\
\text { Disagree }\end{array}$ & Disagree & $\begin{array}{c}\text { Neither } \\
\text { Disagree nor } \\
\text { Agree }\end{array}$ & Agree & Strongly Agree \\
\hline 1 & 2 & 3 & 4 & 5 \\
\hline
\end{tabular}

1. I often use "tips" I hear on how to live a healthy life.

2. I want to use the health information I see every day to live a healthy life.

3. I am motivated to use information that I receive to improve my health.

4. I am motivated to seek information which will improve my health.

5. I am motivated to seek information from people who can help me improve my health.

6. I am interested in seeking information from people who can help me live a healthy life

-_ 7. I am motivated to understand information that will help me live a healthy life.

8. I seek information which helps me maintain a healthy lifestyle.

9. I am interested in understanding how to maintain a healthy lifestyle.

10. I seek information to help me improve my health.

11. I am okay with not understanding everything a healthcare provider is talking about regarding my health. 


\begin{tabular}{|c|c|c|c|c|}
\hline $\begin{array}{c}\text { Strongly } \\
\text { Disagree }\end{array}$ & Disagree & $\begin{array}{c}\text { Neither Disagree } \\
\text { nor Agree }\end{array}$ & Agree & Strongly Agree \\
\hline 1 & 2 & 3 & 4 & 5 \\
\hline
\end{tabular}

12. It is okay with me to not understand all of the words a healthcare provider uses during my visit.

13. I don't need to understand all of the words a healthcare provider uses during my visit.

14. I understand the terms a healthcare provider uses in interactions with me.

15. I understand articles I see on how to be healthy.

16. I understand most of the words people use when they talk about health.

17. When healthcare providers tell me how to treat myself at home (e.g., take medications), I understand how to do so.

18. When people give me suggestions on how to improve my health, I understand what they are trying to tell me and why.

19. I am able to understand discussions about health and healthy living.

20. I know how to seek information from others on how to improve my health.

21. When I talk to a healthcare provider about my health, I am able to understand how I can improve my health.

22. When I talk to a healthcare provider about my health, I am able to understand how to maintain good health.

23. I have a hard time using suggestions I hear on how to live a healthy lifestyle.

24. I worry that healthcare providers will think my questions are "dumb.”

25. I am not able to figure out how to use the instructions a healthcare provider gives me once I am home.

26. I do not use the health suggestions others give me to improve my health. 
Instructions: The following questions concern your current behaviors related to physical activity and healthy eating. For this study, physical activity includes at least 150 minutes of moderate-intensity aerobic physical activity throughout the week or at least 75 minutes of vigorous-intensity aerobic physical activity throughout the week or an equivalent combination of moderate- and vigorous-intensity activity. Healthy eating refers to a diet consisting of low fat and low calorie foods.

Please select ONE of the five options below to best describe your intentions toward physical activity. Circle your response.

(1) No, I do not do the amount of physical activity indicated above, and I do not intend to in the next six months

(2) No, I do not do the amount of physical activity indicated above, but I intend to in the next six months

(3) No, I do not do the amount of physical activity indicated above, but I intend to in the next 30 days

(4) Yes, I regularly do the amount of physical activity indicated above, but I have been doing so for less than six months

(5) Yes, I do the amount of physical activity indicated above, and I have been doing so for more than six months

Please select ONE of the five options below to best describe your intentions toward healthy eating. Circle your response.

(1) No, I do not eat a diet of low fat and low calorie foods, and I do not intend to in the next six months

(2) No, I do not eat a diet of low fat and low calorie foods, but I intend to in the next six months

(3) No, I do not eat a diet of low fat and low calorie foods, but I intend to in the next 30 days

(4) Yes, I do eat a diet of low fat and low calorie foods, but I have been doing so for less than six months

(5) Yes, I do eat a diet of low fat and low calorie foods, and I have been for more than six months 
Please answer the following questions describing yourself. This information will be used for analysis only and will not be used to identify you in any way.

Age:

Sex: Male / Female

Please indicate your marital status (circle your response):

Single Engaged or in a committed relationship Married

Remarried Divorced Widowed

Please indicate how many children you have:

Ethnicity (your dominant ethnic background; circle your response):

Asian

Black/African-American

Hispanic

Native American White/Caucasian

Middle Eastern

Other (specify)

Please indicate your yearly household income(circle your response):

0- $\$ 10,000$

$\$ 10,000-\$ 20,000$

$\$ 20,001-\$ 40,000$

$\$ 40,001-\$ 60,000$

$\$ 60,001-\$ 80,000$

$\$ 80,001-\$ 100,000$

$\$ 100,001-\$ 120,000$

\$120,001-\$140-000

$\$ 140,001+$ 
Please indicate your highest education level (circle your response):

Did not finish high school

High school diploma

General Education Diploma (GED)

College degree (B.A. or B.S.)

Master's degree (M.A. or M.S.)

Doctorate degree

Professional/trade certification

The following question is an indicator of your overall health. If you know your blood glucose level, please write it in the black. If you do not know this information, you may use the glucose meter provided with assistance from the registered LPN on site. This question, like all of the others in this questionnaire, is voluntary.

Blood Glucose Level:

Body Mass Index: 